\title{
Classificação e Discriminação: Um Enfoque Bayesiano
}

\author{
Emerson Wruck
}

Orientador: Prof. Dr. Jorge Alberto Achcar

Dissertação apresentada ao Instituto de Ciências Matemáticas e de Computação - ICMC-USP, como parte dos requisitos para obtenção do título de Mestre em Ciências de Computação e Matemática Computacional.

USP - São Carlos

Fevereiro de 2001 


\section{A Comissão Julgadora:}

Prof. Dr. Jorge Alberto Achcar

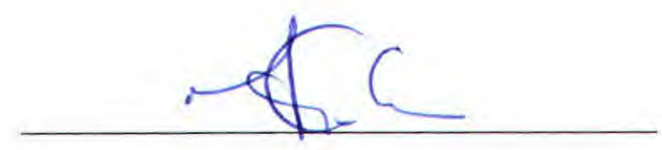

Prof. Dr. Marinho Gomes de Andrade Filho

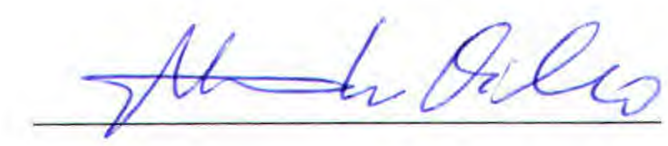

Profa. Dra. Márcia D'Elia Branco

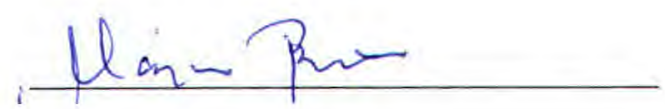




\section{Agradecimentos}

Em primeiro lugar à Deus por me conceder saúde e estar presente sob várias formas em todos os momentos da minha vida.

Ao Prof. Dr. Jorge Alberto Achcar, por me confiar este trabalho, pela orientação segura, compartilhando sua grande experiência e pelo incentivo nas etapas mais difíceis.

Aos professores: Profa. Dra. Maria Creuza B. Salles, Prof.Dr. Josemar Rodrigues e Prof.Dr.Marinho Gomes de Andrade Filho pela dedicação ao ensino e principalmente pela amizade compartilhada.

Ao Prof. Dr. Luiz Milan e ao Prof. Dr. Marinho pelas valiosas sugestões e comentários quando da realização do meu exame de qualificação.

A todos meus amigos que direta ou indiretamente contribuiram para realização deste trabalho, entre eles, Ulisses, Valéria, Vanderly, Lucimary, Josenildo, Sidney, Willian, Manoel, Sandra, Rubia, Val, Gil, Guilherme,Tutia,...

Em especial aos meus padrinhos de casamento e companheiros de turma Cillene e Juliano.

Ao professor e grande amigo Josmar Mazucheli, pela grande ajuda computacional.

Com muito carinho, agradeço a minha família, principalmente pais (Luizinho e Odenilze ) e irmãos (Flávio,Salete, Luis Carlos e Wagner) pelo apoio em todos os aspectos, o incentivo e a confiança.

Com amor agradeço a minha noiva, hoje com muita alegria minha esposa, pelo apoio, compreensão e paciência nos momentos de falta.

Aos funcionários do ICMC pela dedicação e empenho para com todos do Departamento.

E finalmente, à Instituição FAPESP pelo apoio financeiro, sem o qual não teria concluído tal trabalho. 


\begin{abstract}
In this dissertation, we present Bayesian alternatives for classification problem under different approaches.

First of all, we propose a Box and Cox transformation to have normal data to be used in classification problems. We also consider the classification problem assuming a vector $\mathrm{X}$ with a mixture of multivariate normal distributions, using Bayesian procedures to build a classification rule.

We also consider the classification for binary data and correlated binary data using the Bayesian approach and also introducing random effects to capturate the correlation.

For the Bayesian approach, we use MCMC methods and we consider the utilization of the software "Ox" as a great alternative for problems related the efficiency of the algorithm.
\end{abstract}




\section{Sumário}

1 Introdução 1

1.1 Visão geral do problema de Classificação. . . . . . . . . . . . . . . 1

1.1.1 Organização do Trabalho . . . . . . . . . . . . . . 3

2 O problema de Classificação e Discriminação 5

2.1 Visão geral do problema de classificação . . . . . . . . . . . . . 5

2.1.1 Enfoque Bayesiano para o Problema de Classificação . . . . . . . 8

2.2 Classificação de duas populações Normais Multivariadas . . . . . . . . 9

2.3 Desempenho das Funções de Classificação . . . . . . . . . . . . . . 11

2.4 Separação de Populações : Função Discriminante de Fisher. . . . . . . . . . 13

2.5 Classificação de Várias Populações . . . . . . . . . . . . . . . 14

2.5.1 Método do custo mínimo de classificação errada . . . . . . . . 15

2.5.2 Classificação de Populações Normais . . . . . . . . . . . . . 16

2.6 Alguns exemplos ilustrativos. . . . . . . . . . . . . . . . 18

3 Classificação e Discriminação com Dados Transformados 24

3.1 Transformação dos dados para a forma Normal: Caso Univariado. . . . . . 24

3.1.1 Função de Verossimilhança para $\lambda, \mu$ e $\sigma^{2} \ldots \ldots . . . . .25$

3.1.2 Uso do logaritmo da função de verossimilhança perfilada ("Profile Log-Likelihood"). . . . . . . . . . . . . . . . 26

3.2 Transformação de Dados Multivariados (TBox). . . . . . . . . . . . 27

3.3 Enfoque Bayesiano para a transformação Box-Cox (TBox1). . . . . . . . 28

3.3.1 Distribuições a priori para os parâmetros $\lambda_{1}, \lambda_{2}, \ldots, \lambda_{p} \ldots \ldots . . .28$

3.3.2 Distribuição a posteriori conjunta para os parâmetros . . . . . . . . 29 
3.3.3 Distribuições Condicionais a Posteriori necessárias para o algoritmo Gibbs Sampling.

3.4 Outro Enfoque Bayesiano: O uso da Função de Verossimilhança Completa

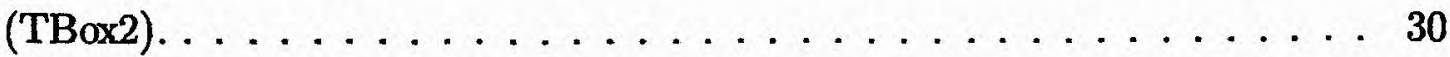

3.4.1 Estimação dos parâmetros de transformação: caso univariado. . . . 30

3.4.2 Estimação dos parâmetros de transformação: caso multivariado. . . 32

3.5 Procedimentos de Simulação e Diagnóstico de Convergência. . . . . . . . . 35

3.6 Alguns Exemplos Numéricos . . . . . . . . . . . . . . . . 35

3.6.1 Estimação dos parâmetros de transformação via Box-Cox Clássico (TBox)

3.6.2 Estimação dos parâmetros de transformação via Box \& Cox Bayesiano

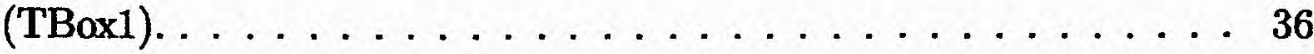

3.6.3 Estimação dos parâmetros de transformação via Box \& Cox Bayesiano

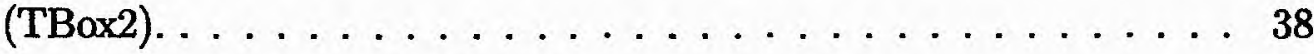

3.6.4 Comparação das transformações e dos procedimentos de Classificação. 40

3.6.5 Resultados para dados transformados de ordem $p>2$. . . . . 42

4 Classificação Considerando a distribuição do vetor de medidas $X$, como uma Misturas de Distribuições

4.1 Introdução aos Modelos de Misturas . . . . . . . . . . . . . . . . . 44

4.2 Análise Bayesiana Assumindo uma Mistura de $K=2$ Distribuições Normais Multivariadas .......................... 45

4.2 .1 Incorporação da Variável Latente . . . . . . . . . . . . . . . . 46

4.3 Distribuições a Priori para o Mòdelo de Mistura . . . . . . . . . . . . . 47

4.3.1 Desenvolvimento do Modelo com Distribuição a Priori Não Informativa ......................... 47

4.3.2 Distribuições Condicionais a Posteriori Necessárias para o Algoritmo Gibbs Sampling . . . . . . . . . . . . . . . . 48

4.3.3 Desenvolvimento do modelo com distribuição a priori conjugada . . 50

4.3.4 Distribuições Condicionais a Posteriori Necessárias para o Algoritmo Gibbs Sampling ................. 51

4.3.5 Classificação para duas Populações . . . . . . . . . . . . . . 53 
4.4 Um Exemplo Пlustrativo . . . . . . . . . . . . . . . . 54

4.4.1 Utilização dos Procedimentos Usuais de Classificação . . . . . . 55

4.5 Aplicação dos Modelos de Mistura para a Classificação . . . . . . . 56

4.5.1 Utilização de Distribuiçōes a Priori Não Informativas . . . . . . 57

4.5.2 Utilização de Distribuições a Priori Informativas . . . . . . . . 61

4.5.3 Classificação Utilizando o algoritmo EM . . . . . . . 64

5 Classificação e Discriminação a partir de Variáveis Binárias 67

5.1 Desenvolvimento do Critério de Classificação $\ldots \ldots \ldots \ldots$

5.2 Alternativa Bayesiana para a Classificação Binária. . . . . . . . . 70

5.3 Exemplo de Aplicação. . . . . . . . . . . . . . 72

6 Classificação Considerando Dados Binários Correlacionados $\quad 79$

6.1 Desenvolvimento do Modelo . . . . . . . . . . . . . . 79

6.2 Análise Bayesiana Hierárquica Assumindo Efeitos Aleatórios Normais . . 80

6.3 Modelos de Efeitos Aleatórios com uma Mistura de Distribuições Normais . 84

6.4 Exemplo Пlustrativo . . . . . . . . . . . . . . . . 89

7 Conclusōes Finais e Propostas Futuras 100

$\begin{array}{ll}\text { Apêndices } & 102\end{array}$

A ALGORITMO GIBBS SAMPLING 102

B ALGORITMO METROPOLIS-HASTINGS 104

C O CRITÉRIO DE CONVERGÊNCIA DE GELMAN E RUBIN (1992) 106

D O CRITÉRIO DE CONVERGÊNCIA DE GEWEKE (1992) 108

E UM PROGRAMA COMPUTACIONAL 110

$\begin{array}{ll}\text { Referências Bibliográficas } & 115\end{array}$ 


\section{Capítulo 1}

\section{Introdução}

\subsection{Visão geral do problema de Classificação.}

Num problema de classificação, temos um objeto (ou pessoa) conhecido que pertence a um de $g$ grupos distintos de um mesmo universo. O objetivo é designar o objeto a um desses grupos com base num vetor $\mathrm{x}$ de medidas características associado ao objeto(ver por exemplo Cacoullos,1973; Lachenbruch,1975a; Huberty,1975; Goldstein e Dillon,1978; Lachenbruch \& Goldstein,1979; Johnson \& Wichern,1982). Este problema é verificado em diversas áreas de aplicações tais como Ciências Sociais, Economia, Medicina, Biologia, Física, etc.

Algumas situações em que necessitamos usar classificação são ilustradas abaixo:

a- Conhecimento incompleto ou desempenho futuro. Ex: No passado valores extremos de certas variáveis financeiras foram observadas dois anos antes de uma falência. A classificação de uma outra firma como segura ou em dificuldades, com base nos valores observados desses indicadores, pode permitir que os executivos tomem a ação corretiva (quando necessária), antes que seja muito tarde.

b- Informação perfeita exige a destruição do objeto. Ex: Tempo de vida de uma bateria é determinado usando até acabar. Produtos sem carga não podem ser vendidos. Poderíamos classificar produtos como bons ou ruins com base em certas medidas preliminares.

c- Informação impossível ou muito cara. Ex: Atribuir autoria de artigos. Freqüências das palavras ou comprimento das sentenças podem ajudar a classificar a autoria. 
d- O número de trabalhos literários datados por um autor estão disponíveis e estes podem ser classificados em $g$ grupos cronologicamente. Um trabalho não datado é encontrado e desejamos classifica-lo cronologicamente. Aqui devemos mensurar o estilos característicos que mudam com o tempo.

Em geral nas abordagens usadas para o problema de classificação, (ver por exemplo Anderson,1984; Johnson \& Wichern,1982), assume-se que o vetor aleatório X de medidas associado a cada objeto, tenha um distribuição Normal Multivariada e usa-se os procedimentos tradicionais de classificação como a Função Discriminante Linear (F.D.L) e a Função Discriminante Quadrática.(F.D.Q). No entanto, quando não temos a normalidade multivariada para a distribuição de $\mathrm{X}$, podemos ter problemas na taxa dè classificação de novos objetos, usando tais procedimentos.

Neste trabalho exploramos os procedimentos de Inferência Bayesiana, aplicados direta e indiretamente ao problema de classificação e discriminação, utilizando principalmente os métodos MCMC (Monte Carlo em Cadeias Markov); entre esses métodos, destacamos o algoritmo Gibbs Sampling (ver por exemplo, Gelfand \& Smith, 1990) e o algoritmo Metropolis-Hastings (ver por exemplo Smith \& Roberts,1993).

Na primeira parte do trabalho, considèrando uma distribuição contínua para o vetor aleatório $\mathrm{X}$ de medidas, exploramos o problema de classificação e discriminação utilizando a transformação de Box \& Cox (1964): Aqui, transformamos os dados para uma distribuição Normal Multivariada e usamos as técnicas usuais de discriminação (F.D.L e F.D.Q). Assim, consideramos uma transformação Box-Cox para cada variável na forma:

$$
x_{i}^{\left(\lambda_{i}\right)}= \begin{cases}\frac{x_{i}^{\lambda_{i}}-1}{\lambda_{i}} & \text { se } \lambda_{i} \neq 0, \\ \ln \left(x_{i}\right) & \text { se } \lambda_{i}=0 .\end{cases}
$$

onde $i=1, \ldots, k ; \quad \mathrm{x}=\left(x_{1}, x_{2}, \ldots, x_{k}\right)$.

Quando temos $\mathrm{x}$ de dimensão alta, $k \geq 3$, a obtenção dos valores de $\lambda_{i}$ pelo método clássico "Profile Log-Likelihood" pode se tornar complicado( ver Johnson \& Wichern,pp 167-171), necessitando de muito trabalho computacional. O uso de algoritmos MCMC citados acima pode ser uma alternativa razoável para achar os valores de $\lambda_{i}, i=1,2, \ldots, k$, a partir da função de verossimilhança perfilada. Também utilizamos procedimentos Bayesianos para estimar os valores acima, diretamente da função de verossimilhança completa. 
Outra ênfase dada para o problema de classificação, foi supor para o vetor aleatório $\mathrm{X}$ de medidas, uma distribuição diferente da distribuição Normal Multivariada. Neste caso, consideramos uma mistura de distribuições normais multivariadas (ver por exemplo Robert,1996; Lavine \& West,1992) que em algumas situações, possibilitam uma męlhor adequação do modelo aos dados e usamos a distribuição preditiva na regra de classificação.

Consideramos também, o problema de classificação a partir de dados binários. Añalisamos o procedimento usual para a classificação e propusemos procedimentos alternativos Bayesianos, onde incorporamos o fator correlação com o intuito de formular um modelo mais robusto.

\subsubsection{Organização do Trabalho}

Este trabalho encontra-se dividido em 7 capítulos. Neste capítulo introdutório apresentamos uma idéia geral do problema de Classificação e Discriminação, citando alguns exemplos cotidianos e um pequeno esboço do que se refere o trabalho.

No capítulo 2, apresentamos a maioria dos conceitos e procedimentos usuais do problema de Classificação e Discriminação considèrando dados contínuos e utilizamos exemplos ilustrativos de alguns livros textos ( por exemplo, Applied Multivariate Statistical Analysis,Johnson \& Wichern, 1982; Statistical Methods for Survival Data Analysis, Lee, 1980).

No capítulo 3, consideramos a transformação de Box \& Cox para os dados dos exemplos mostrados no capítulo 2. Aqui utilizamos procedimentos Bayesianos para a transformação dos dados e aplicamos as regras usuais de classificação utilizadas anteriormente. Todos os resultados foram analisados e discutidos.

No capítulo 4, apresentamos modelos com misturas de distribuições normais multivariadas, considerando a introdução de variáveis latentes. Dentro do enfoque Bayesiano, utilizamos o algoritmo Gibbs Sampling para estimação e consideramos ainda para o problema distribuições a priori não informativas e informativas conjugadas. Corno alternativa Clássica, utilizamos o algoritmo EM (ver McLachlan \& Krishnan, 1997). A classificação foi feita com base na densidade preditiva para cada população.

No capítulo 5, apresentamos o problema de Classificação e Discriminação utilizando somente variáveis binárias. Neste caso, introduzimos o procedimento usual de classificação e uma opção Bayesiana. 
No capítulo 6, dando continuidade ao problema de classificação com dados binários, foi proposto modelos que incorporam o fator correlação entre as variáveis binárias associadas a cada objeto.

No capítulo 7, apresentamos as conclusões e algumas propostas futuras. 


\section{Capítulo 2}

\section{O problema de Classificação e Discriminação}

Neste capítulo apresentamos uma breve introdução ao problema de classificação e discriminação dentro da abordagem tradicional.

\subsection{Visão geral do problema de classificação}

Inicialmente nos concentramos no problema de classificação para duas populações embora a maioria das idéias apresentadas possam ser imediatamente generalizadas para mais de duas populações.

Sejam $f_{1}(\mathbf{x})$ e $f_{2}(\mathbf{x})$, as funções densidades de probabilidades associadas com o vetor da v.a $\mathrm{X}_{(p \times 1)}$ para as populações $\pi_{1}$ e $\pi_{2}$, respectivamente. Um objeto com medidas associadas $\mathrm{x}$, deve ser atribuído à $\pi_{1}$ ou $\pi_{2}$. Seja $\Omega$, o espaço amostral, isto é, a coleção de todas as observações possíveis de $\mathrm{x}$. Temos $R_{1}$ : o conjunto de valores de $\mathrm{x}$, utilizados para classificar objetos como $\pi_{1}$ e $R_{2}=\Omega-R_{1}$ : os valores restantes utilizados para classificar $\pi_{2}$; aqui os conjuntos $R_{1}$ e $R_{2}$ são mutuamente exclusivos.

A probabilidade condicional, $P(2 \mid 1)$, de classificar um objeto como $\pi_{2}$, quando de fato é de $\pi_{1}$ é dada por,

$$
P(2 \mid 1)=P\left(\mathrm{x} \in R_{2} \mid \pi_{1}\right)=\int_{R_{2}=\Omega-R_{1}} f_{1}(\mathrm{x}) d \mathrm{x}
$$

Similarmente, a probabilidade condicional, $P(1 \mid 2)$, de classificar um objeto como $\pi_{1}$, quan- 
do na verdade é $\pi_{2}$ é dada por,

$$
P(1 \mid 2)=P\left(\mathrm{x} \in R_{1} \mid \pi_{2}\right)=\int_{R_{1}} f_{2}(\mathrm{x}) d \mathrm{x}
$$

Agora, sejam $p_{1}$, a probabilidade a priori de $\pi_{1}$ e $p_{2}$, a probabilidade a priori de $\pi_{2}$, onde $p_{1}+p_{2}=1$. As probabilidades totais de classificar objetos corretamente ou incorretamente podem ser derivadas dos produtos das probabilidades a priori e probabilidades condicionais.

$$
\begin{gathered}
P\left(\text { classificada corretamente como } \pi_{1}\right)=P\left(\mathrm{X} \in R_{1} \mid \pi_{1}\right) P\left(\pi_{1}\right) \\
P\left(\text { classificada incorretamente como } \pi_{1}\right)=P\left(\mathrm{X} \in R_{1} \mid \pi_{2}\right) P\left(\pi_{2}\right) \\
P\left(\text { classificada corretamente como } \pi_{2}\right)=P\left(\mathrm{X} \in R_{2} \mid \pi_{2}\right) P\left(\pi_{2}\right) \\
P\left(\text { classificada incorretamente como } \pi_{2}\right)=P\left(\mathrm{X} \in R_{2} \mid \pi_{1}\right) P\left(\pi_{1}\right)
\end{gathered}
$$

Assim, mesmo uma probabilidade aparentemente pequena pode ser muito grande se o custo de fazer uma atribuição incorreta for muito alto. O custo de uma classificação errada pode ser definido por uma matriz de custo (ver Tabela 2.1).

Tabela 2.1: Matriz de Custo

\begin{tabular}{c|c|cc}
\multicolumn{3}{c}{ Classificar como } \\
\hline & & $\pi_{1}$ & $\pi_{2}$ \\
\hline População & $\pi_{1}$ & 0 & $c(2 \mid 1)$ \\
Verdadeira & $\pi_{2}$ & $c(1 \mid 2)$ & 0 \\
\hline
\end{tabular}

onde

$c(1 \mid 2)$ : custo da observação de $\pi_{2}$ ser incorretamente classificada em $\pi_{1}$.

$c(2 \mid 1)$ : custo da observação de $\pi_{1}$ ser incorretamente classificada em $\pi_{2}$.

O custo esperado por classificação errada (ECM) é dado por,

$$
E C M=c(2 \mid 1) P(2 \mid 1) p_{1}+c(1 \mid 2) P(1 \mid 2) p_{2}
$$

O objetivo para uma boa regra de classificação é obter um ECM com valor bem pequeno.

Resultado 2.1. : As regiões $R_{1}$ e $R_{2}$ que minimizam o ECM são definidas pelos valores de $x$ para os quais as seguintes desigualdades valem. 


$$
R_{1}: \frac{f_{1}(x)}{f_{2}(x)} \geq\left[\frac{c(1 \mid 2)}{c(2 \mid 1)}\right]\left[\frac{p_{2}}{p_{1}}\right] \quad R_{2}: \frac{f_{1}(x)}{f_{2}(x)}<\left[\frac{c(1 \mid 2)}{c(2 \mid 1)}\right]\left[\frac{p_{2}}{p_{1}}\right]
$$

Prova:(ver Johnson \& Wichern,1982; Seber,1983)

Alguns casos especiais de regiões de custos mínimos esperados, são dados por:

a) $\left(p_{1} / p_{2}\right)=1 \quad$ (probabilidades a priori iguais)

$$
R_{1}: \frac{f_{1}(\mathrm{x})}{f_{2}(\mathrm{x})} \geq\left[\frac{c(1 \mid 2)}{c(2 \mid 1)}\right] \quad R_{2}: \frac{f_{1}(\mathrm{x})}{f_{2}(\mathrm{x})}<\left[\frac{c(1 \mid 2)}{c(2 \mid 1)}\right]
$$

b) $c(1 \mid 2) / c(2 \mid 1)=1 \quad$ ( custos de classificação erradas iguạis)

$$
R_{1}: \frac{f_{1}(\mathrm{x})}{f_{2}(\mathrm{x})} \geq\left[\frac{p_{2}}{p_{1}}\right] \quad R_{2}: \frac{f_{1}(\mathrm{x})}{f_{2}(\mathrm{x})}<\left[\frac{p_{2}}{p_{1}}\right]
$$

c) $\left(p_{1} / p_{2}\right)=(c(1 \mid 2) / c(2 \mid 1))=1$

$$
R_{1}: \frac{f_{1}(\mathrm{x})}{f_{2}(\mathrm{x})} \geq 1 \quad R_{2}: \frac{f_{1}(\mathrm{x})}{f_{2}(\mathrm{x})}<1
$$

Em geral, sendo as probabilidades a priori desconhecidas, estas são consideradas iguais. Também se é desconhecida a razão entre os custos de uma classificação errada, então ela pode ser considerada igual a 1 .

Quando ignorarmos os custos de classificação errada, podemos escolher $R_{1}$ e $R_{2}$ que minimizam a probabilidade total de classificação errada TPM.

$$
T P M=p_{1} \int_{R_{2}} f_{1}(\mathrm{x}) d \mathrm{x}+p_{2} \int_{R_{1}} f_{2}(\mathrm{x}) d \mathrm{x}
$$

Observar que este problema é equivalente a minimizar o custo esperado de classificação errada quando os custos de classificação são iguais. Portanto as regiōes ótimas neste caso são dados no item (b) acima. Podemos também alocar uma nova observação $x_{0}$ para a população com maior probabilidade a posteriori $P\left(\pi_{i} \mid \mathrm{x}_{0}\right)$, onde pela regra de Bayes as probabilidades a posteriori são dadas por,

$$
\begin{aligned}
& P\left(\pi_{1} \mid \mathrm{x}_{0}\right)=\frac{p_{1} f_{1}\left(\mathrm{x}_{0}\right)}{p_{1} f_{1}\left(\mathrm{x}_{0}\right)+p_{2} f_{2}\left(\mathrm{x}_{0}\right)} \\
& P\left(\pi_{2} \mid \mathrm{x}_{0}\right)=\frac{p_{2} f_{2}\left(\mathrm{x}_{0}\right)}{p_{1} f_{1}\left(\mathrm{x}_{0}\right)+p_{2} f_{2}\left(\mathrm{x}_{0}\right)}
\end{aligned}
$$

Classificar uma observação $\mathrm{x}_{0}$ como $\pi_{1}$ quando $P\left(\pi_{1} \mid \mathrm{x}_{0}\right) \geq P\left(\pi_{2} \mid \mathrm{x}_{0}\right)$ é equivalente a usar a regra do item (b) acima, pois os denominadores são os mesmos. 


\subsubsection{Enfoque Bayesiano para o Problema de Classificação}

Nesta seção, vamos continuar nos concentrando em classificação para duas populações embora a maioria das idéias apresentadas possam ser imediatamente generalizadas para mais de 2 populações.

Supor agora as distribuições $f_{j}\left(\mathbf{x} \mid \boldsymbol{\theta}_{j}\right)$ parametrizadas pelo vetor $\boldsymbol{\theta}_{\boldsymbol{j}}, \operatorname{com} j=1,2$ para cada respectiva população $\pi_{1}$ e $\pi_{2}$. Sob o enfoque Bayesiano, vamos ter para cada $\theta_{j}$ uma distribuição a priori $g_{j}\left(\boldsymbol{\theta}_{j}\right)$. Conseqüentemente, teremos também, para cada $j=1,2$ uma distribuição do vetor de parâmetros dado os dados $g_{j}\left(\boldsymbol{\theta}_{j} \mid \mathrm{x}\right)$,designada como distribuição a posteriori, que pode ser escrita como:

$$
g_{j}\left(\boldsymbol{\theta}_{j} \mid \mathbf{x}\right) \propto g_{j}\left(\boldsymbol{\theta}_{j}\right) f_{j}\left(\mathbf{x} \mid \boldsymbol{\theta}_{j}\right)
$$

Podemos então determinar as densidades preditivas para cada população $j=1,2$ por:

$$
\begin{aligned}
& f_{1}(\mathbf{x})=\int_{\Theta_{1}} f_{1}\left(\mathbf{x} \mid \boldsymbol{\theta}_{1}\right) g_{1}\left(\boldsymbol{\theta}_{1} \mid \mathbf{x}\right) d \boldsymbol{\theta}_{1} \\
& f_{2}(\mathbf{x})=\int_{\Theta_{2}} f_{2}\left(\mathbf{x} \mid \boldsymbol{\theta}_{2}\right) g_{2}\left(\boldsymbol{\theta}_{2} \mid \mathbf{x}\right) d \boldsymbol{\theta}_{2}
\end{aligned}
$$

onde $\theta_{j}, j=1,2$ é o espaço de parâmeţros.

Assim determinamos as regiōes de classificação de acordo com o Resultado 1.1, onde temos,

$$
R_{1}: \frac{f_{1}(\mathrm{x})}{f_{2}(\mathrm{x})} \geq\left[\frac{c(1 \mid 2)}{c(2 \mid 1)}\right]\left[\frac{p_{2}}{p_{1}}\right] \quad R_{2}: \frac{f_{1}(\mathrm{x})}{f_{2}(\mathrm{x})}<\left[\frac{c(1 \mid 2)}{c(2 \mid 1)}\right]\left[\frac{p_{2}}{p_{1}}\right]
$$

Aqui, podemos usar métodos de simulação via MCMC (Monte Carlo em Cadeias de Markov), para gerar amostras para $\theta_{j}, j=1,2$. Gerado uma amostra, usamos estimativas de $f_{1}(\mathrm{x})$ e $f_{2}(\mathrm{x})$ para realizarmos o procedimento de classificação. As estimativas de Monte Carlo para $f_{1}(\mathrm{x})$ e $f_{2}(\mathrm{x})$ são dadas por:

$$
\begin{aligned}
& \hat{f}_{1}(\mathbf{x})=\frac{1}{M} \sum_{s=1}^{M} f_{1}\left(\mathbf{x} \mid \theta_{1}^{(s)}\right) \\
& \hat{f}_{2}(\mathbf{x})=\frac{1}{M} \sum_{s=1}^{M} f_{2}\left(\mathbf{x} \mid \theta_{2}^{(s)}\right)
\end{aligned}
$$

onde $M$ é o número de valores gerados para cada parâmetro na amostra, $s=1, \ldots, M$.

Usualmente assumimos $f_{1}\left(\mathbf{x} \mid \boldsymbol{\theta}_{1}\right)$, onde $\boldsymbol{\theta}_{1}=\left(\boldsymbol{\mu}_{1}, \boldsymbol{\Sigma}_{1}\right)$ e $f_{2}\left(\mathbf{x} \mid \boldsymbol{\theta}_{2}\right)$, onde $\boldsymbol{\theta}_{2}=\left(\boldsymbol{\mu}_{2}, \boldsymbol{\Sigma}_{2}\right)$ com distribuições Normais $p$-variada $N_{p}\left(\mu_{1}, \Sigma_{1}\right)$ e $N_{p}\left(\mu_{2}, \Sigma_{2}\right)$. Num caso mais geral podemos supor que $f_{1}\left(\mathbf{x} \mid \boldsymbol{\theta}_{1}\right)$ e $f_{2}\left(\mathbf{x} \mid \boldsymbol{\theta}_{2}\right)$ tenham distribuições $t$-Student $p$-variądas. 


\subsection{Classificação de duas populaçẽes Normais Multi-}

\section{variadas}

Supor que $f_{1}(\mathrm{x})$ e $f_{2}(\mathrm{x})$ sejam densidades normais multivariadas, a primeira com vetor de média $\mu_{1}$ e matriz de covariância $\Sigma_{1}$ e a segunda com vetor de média $\mu_{2}$ e matriz de covariância $\boldsymbol{\Sigma}_{2}$. Num caso especial, considerar também que $\boldsymbol{\Sigma}_{1}=\boldsymbol{\Sigma}_{\mathbf{2}}=\mathbf{\Sigma}$.

Seja a densidade conjunta de $\mathrm{X}^{\prime}=\left[X_{1}, X_{2}, \ldots, X_{p}\right]$ para as populaçōes $\pi_{1}$ e $\pi_{2}$ dạdas por :

$$
f_{i}(\mathbf{x})=\frac{1}{(2 \pi)^{p / 2}|\Sigma|^{1 / 2}} \exp \left[-\frac{1}{2}\left(\mathbf{x}-\mu_{i}\right)^{\prime} \Sigma^{-1}\left(\mathbf{x}-\mu_{i}\right)\right]
$$

para $i=1,2$.

Supor que os parâmetros populacionais $\mu_{1}, \mu_{2}$ e $\boldsymbol{\Sigma}$ sejam conhecidos.

Neste caso, as regiões que minimizam o ECM são dadas por,

$$
\begin{aligned}
& R_{1}: \frac{f_{1}(\mathrm{x})}{f_{2}(\mathrm{X})}=\exp \left\{-\frac{1}{2}\left(\mathrm{x}-\mu_{1}\right)^{\prime} \boldsymbol{\Sigma}^{-1}\left(\mathrm{x}-\mu_{1}\right)+\frac{1}{2}\left(\mathrm{x}-\mu_{2}\right)^{\prime} \Sigma^{-1}\left(\mathrm{x}-\mu_{2}\right)\right\} \geq\left[\frac{c(1 \mid 2)}{c(2 \mid 1)}\right]\left[\frac{p_{2}}{p_{1}}\right] \\
& R_{2}: \frac{f_{1}(\mathrm{X})}{f_{2}(\mathrm{X})}=\exp \left\{-\frac{1}{2}\left(\mathrm{x}-\mu_{1}\right)^{\prime} \mathbf{\Sigma}^{-1}\left(\mathrm{x}-\mu_{1}\right)+\frac{1}{2}\left(\mathrm{x}-\mu_{2}\right)^{\prime} \Sigma^{-1}\left(\mathrm{x}-\mu_{2}\right)\right\}<\left[\frac{c(1 \mid 2)}{c(2 \mid 1)}\right]\left[\frac{p_{2}}{p_{1}}\right]
\end{aligned}
$$

Resultado 2.2. : Sejam as populaçôes $\pi_{1}$ e $\pi_{2}$ descritas por (2.11). A regra de alocação que minimiza o ECM é dada por:

Alocar $x_{0} e m \pi_{1}$ se

$$
\left(\mu_{1}-\mu_{2}\right)^{\prime} \Sigma^{-1} x_{0}-\frac{1}{2}\left(\mu_{1}-\mu_{2}\right)^{\prime} \Sigma^{-1}\left(\mu_{1}+\mu_{2}\right) \geq \ln \left[\frac{c(1 \mid 2)}{c(2 \mid 1)} \frac{p_{2}}{p_{1}}\right]
$$

caso contrário, alocar $x_{0} e m \pi_{2}$.

Prova: (Johnson \& Wichern,198̧2).

Na maioria das situações, as quantidades populacionais $\boldsymbol{\mu}_{1}, \boldsymbol{\mu}_{2}$ e $\boldsymbol{\Sigma}$ são desconhecidas. Neste caso, alguns autores (ver por exemplo Anderson,T. W.,1984) sugerem a regrą de alocação que minimiza o ECM para duas populações normais dada por:

Alocar $\mathrm{x}_{0}$ em $\pi_{1}$ se

$$
\left(\overline{\mathbf{x}}_{1}-\overline{\mathbf{x}}_{2}\right)^{\prime} S_{\text {pooled }}^{-1} \mathrm{x}_{0}-\frac{1}{2}\left(\overline{\mathbf{x}}_{1}-\overline{\mathbf{x}}_{2}\right)^{\prime} S_{\text {pooled }}^{-1}\left(\overline{\mathbf{x}}_{1}+\overline{\mathbf{x}}_{2}\right) \geq \ln \left[\frac{c(1 \mid 2)}{c(2 \mid 1)} \frac{p_{2}}{p_{1}}\right]
$$


caso contrário alocar $\mathrm{x}_{0}$ em $\pi_{2}$.

Neste caso, estamos supondo que temos, $n_{1}$ observações de uma variável aleatória multivariada $\mathrm{X}^{\prime}=\left[X_{1}, X_{2}, \ldots, X_{p}\right]$ de $\pi_{1}$ e $n_{2}$ medidas desta quantidade para $\pi_{2}$, com $n_{1}+n_{2}-2 \geq p$. As respectivas matrizes de dados são:

$$
\begin{aligned}
& X_{1}=\left[x_{11}, x_{12}, \ldots, x_{1 n_{1}}\right]_{\left(p \times n_{1}\right)} \\
& X_{2}=\left[x_{21}, x_{22}, \ldots, x_{2 n_{2}}\right]_{\left(p \times n_{2}\right)}
\end{aligned}
$$

Para essas matrizes de dados, os vetores de médias e a matrizes de covariância são dados por,

$$
\begin{aligned}
& \bar{x}_{i}=\frac{1}{n_{i}} \sum_{j=1}^{n_{i}} x_{i j} \\
& S_{i}=\frac{1}{n_{i}-1} \sum_{j=1}^{n_{i}}\left(x_{i j}-\bar{x}_{i}\right)\left(x_{i j}-\bar{x}_{i}\right)^{\prime}
\end{aligned}
$$

onde $i=1,2$

Supondo $\mathbf{\Sigma}$ comum, temos,

$$
S_{\text {pooled }}=\frac{\left(n_{1}-1\right) S_{1}+\left(n_{2}-1\right) S_{2}}{\left(n_{1}+n_{2}-2\right)}
$$

A expressão

$$
w=\left(\overline{\mathbf{x}}_{1}-\overline{\mathbf{x}}_{2}\right)^{\prime} \mathbf{S}_{\text {pooled }}^{-1} \mathrm{x}_{0}-\frac{1}{2}\left(\overline{\mathbf{x}}_{1}-\overline{\mathbf{x}}_{2}\right)^{\prime} \mathbf{S}_{\text {pooled }}^{-1}\left(\overline{\mathbf{x}}_{1}+\overline{\mathbf{x}}_{2}\right)
$$

é conhecida como função (estatística) de classificaçãa de Anderson ou Função Discriminante Linear (F.D.L).

Quando $\boldsymbol{\Sigma}_{1} \neq \boldsymbol{\Sigma}_{2}$, consideranda as densidades normais multivariadas, dadas em (2.11) corn $\Sigma_{i}, i=1,2$, no lugar de $\Sigma$, tepros:

$$
\begin{aligned}
& R_{1}=-\frac{1}{2} \mathrm{x}^{\prime}\left(\Sigma_{1}^{-1}-\Sigma_{2}^{-1}\right) \mathrm{x}+\left(\mu_{1}^{\prime} \Sigma_{1}^{-\mathrm{I}}-\mu_{2}^{\prime} \Sigma_{2}^{-1}\right) \mathrm{x}-k \geq \ln \left[\frac{c(1 \mid 2)}{c(2 \mid 1)} \frac{p_{2}}{p_{1}}\right] \\
& R_{2}=-\frac{1}{2} \mathrm{x}^{\prime}\left(\Sigma_{1}^{-1}-\Sigma_{2}^{-1}\right) \mathrm{x}+\left(\mu_{1}^{\prime} \Sigma_{1}^{-1}-\mu_{2}^{\prime} \Sigma_{2}^{-1}\right) \mathrm{x}-k<\ln \left[\frac{c(1 \mid 2)}{c(2 \mid 1)} \frac{p_{2}}{p_{1}}\right]
\end{aligned}
$$

onde

$$
k=\frac{1}{2} \ln \left(\frac{\left|\Sigma_{1}\right|}{\left|\Sigma_{2}\right|}\right)+\frac{1}{2}\left(\mu_{1}^{\prime} \Sigma_{1}^{-1} \mu_{1}-\mu_{2}^{\prime} \Sigma_{2}^{-1} \mu_{2}\right)
$$

As regiões de classificação são definidas pelas funções quadráticas de $\mathbf{x}$. 
Resultado 2.3. : Sejam as populações $\pi_{1}$ e $\pi_{2}$ descritas por densidades normais multivariadas com vetor de médias e matriz de covariância, respectivamente $\boldsymbol{\mu}_{1}, \mathbf{\Sigma}_{1}$ e $\boldsymbol{\mu}_{\mathbf{2}}, \boldsymbol{\Sigma}_{\mathbf{2}}$. A regra de classificaçâo que minimiza o custo esperado da classificação errada é dada por: Alocar $x_{0}$ em $\pi_{1}$ se

$$
-\frac{1}{2} x_{0}^{\prime}\left(\Sigma_{1}^{-1}-\Sigma_{2}^{-1}\right) x_{0}+\left(\mu_{1}^{\prime} \Sigma_{1}^{-1}-\mu_{2}^{\prime} \Sigma_{2}^{-1}\right) x_{0}-k \geq \ln \left[\frac{c(1 \mid 2)}{c(2 \mid 1)} \frac{p_{2}}{p_{1}}\right]
$$

caso contrário, alocar $x_{0}$ em $\pi_{2}$

Na prática, $\boldsymbol{\mu}_{1}, \boldsymbol{\Sigma}_{1}$ e $\boldsymbol{\mu}_{2}, \boldsymbol{\Sigma}_{2}$, são substituídos pelas respectivas quantìdadès amostrais $\overline{\mathbf{x}}_{1}, \overline{\mathbf{x}}_{2}, \mathbf{S}_{1}$ e $\mathbf{S}_{2}$. Portanto, a regra de classificação quádrática ou Função Discriminante Quadrática (F.D.Q) é dadà por:

Alocar $\mathrm{x}_{0}$ em $\pi_{1}$ se

$$
-\frac{1}{2} \mathrm{x}_{0}^{\prime}\left(\mathrm{S}_{1}^{-1}-\mathrm{S}_{2}^{-1}\right) \mathrm{x}_{0}+\left(\overline{\mathrm{x}}_{1}^{\prime} \mathrm{S}_{1}^{-1}-\overline{\mathrm{x}}_{2}^{\prime} \mathrm{S}_{2}^{-1}\right) \mathrm{x}_{0}-k \geq \ln \left[\frac{c(1 \mid 2)}{c(2 \mid 1)} \frac{p_{2}}{p_{1}}\right]
$$

caso contrário, alocar $\mathrm{x}_{0}$ em $\pi_{2}$.

\subsection{Desempenho das Funções de Classificação}

Uma maneira de julgar o desempenho de qualquer procedimento de classificação é calcular suas "taxas de erros" ou probabilidades de classificação erradas. Esses valores podem ser obtidos facilmente, quando as populações são conhecidas. No entanto, na prática isso ocorre raramente, e precisamos trábalhar com taxas de erros associados a função de classificação amostral. Esta função de classificação é construída, baseada no seu desempenho em amostras futuras.

A probabilidade total de classificação errada (TPM) é dado por:

$$
T P M=p_{1} \int_{R_{2}} f_{1}(\mathrm{x}) \mathrm{dx}+p_{2} f_{R_{1}} f_{2}(\mathrm{x}) d \mathrm{x}
$$

$O$ menor valor dessa quantidade é obtido pela escolha adequada de $R_{1}$ e $R_{2}$ (supondo custos de classificação erradas iguais), e é chamado de taxa de erro ótimo (OER).

Uma medida de desempenho que não depende das formas das populações e podem ser calculadas para qualquer procedimento de classificação, é dada pela taxa de erro aparente $(A P E R)$, definida como a fração de observações na amostra piloto que são classificadas incorretamente pela função de classificação amostral. Para $n_{1}$ observações de $\pi_{1}$ e $n_{2}$ observações de $\pi_{2}$, temos a Tabela de classificação 2.2 abaixo: 
Tabela 2.2: Tabela de Classificação

\begin{tabular}{c|c|c|c}
\multicolumn{3}{c}{ Membro pela Previsão } \\
\hline & & $\pi_{1}$ & $\pi_{2}$ \\
\hline Membro & $\pi_{1}$ & $n_{1 C}$ & $n_{1 M}=n_{1}-n_{1 C}$ \\
Verdadeiro & $\pi_{2}$ & $n_{2 M}=n_{2}-n_{2 C}$ & $n_{2 C}$ \\
\hline
\end{tabular}

onde

$n_{i C}$ : número de itens de $\pi_{i}$ classsificados corretamente comp $\pi_{i}$.

$n_{i M}$ : número de itens dè $\pi_{i}$ clàssificados incorretamentè como $\pi_{j}$.

A taxa de erro aparente é então:

$$
A P E R=\frac{n_{1 M}+n_{2 M}}{n_{1}+n_{2}}
$$

que é conhecida como proporção de itens na amostra piloto que são classificados incorretamente.

Nà prática podemos dividir uma amostra em duas sub-amostras: uma amostra piloto (treino) para construir a função de classificação e outra para verificar o desèmpenho da função. Neste caso temos dois problemas:

(i) Este procedimento requer uma amostra grande.

(ii) A função avaliada não é a de interesse Quase todós os dados devem ser considerados para construir a função de classificação, caso contráriò o valor dả informação pode não ser muito bom.

Outra técnica que parece füncionar bèm é o procedimento "holdout"de Lachenbruch (ver Lachenbruch.\& Mickey,1968).

1. Iniciar com o grupo dè observaçôes de $\pi_{1}$. Omita uma observação dèsse grupo e desenvolva uma função de classificação baseada nas $n_{1}-1$ observações restantes.

2. Classifique essa observação omitidà usando a funçãa construída na etapa 1 .

3. Repita passos 1 e 2 até classificar todas as observaçōes. Seja $n_{1 M}^{I}$ : a número de holdout (H) observações classificadas incorretamente neste grupo.

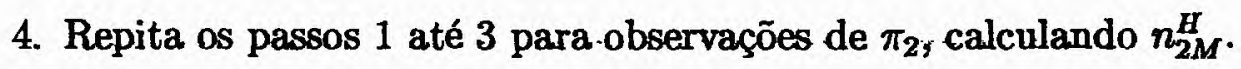


As estimativas de $\hat{P}(2 \mid 1)$ e $\hat{P}(1 \mid 2)$ são dadas por,

$$
\hat{P}(2 \mid 1)=\frac{n_{1 M}^{(H)}}{n_{1}} \quad \text { e } \quad \hat{P}(1 \mid 2)=\frac{n_{2 M}^{(H)}}{n_{2}}
$$

e a.estimativa da taxa de erros esperadoE(AER) é dadâ.por:

$$
\hat{E}(A E R)=\frac{n_{1 M}^{(H)}+n_{2 M}^{(H)}}{n_{1}+n_{2}}
$$

\section{2:4 Separação dè Populações : Função Discriminante de-Fisher.}

A idéia de Fisher (Fisher,R-A, 1936) é transformar as observações multivariadas xy èm observaçōès univariadas $y$, ondè $y^{\prime} s$ provêm dàs populações $\pi_{1}$ e $\pi_{2}$, separadàs o máximo possível (aqui estamos considerando separação de duas populações).

O procedìmento de Fisher não assume que as populaçōes sejam normais. No entanto, assume-se que as matrizes de covariâncias populacionais sejam iguais.

Uma combinação linear fixada para os x's tomam os vatores $y_{11}, y_{12}, \ldots, y_{1 n_{1}}$ para as observações da população $\pi_{1}$ e os valores $y_{21}, y_{22}, \ldots, y_{2 n_{2}}$ para as observações da população $\pi_{2}$. A separação desses dois conjuntos univariadòs $y^{\prime} s$ é feita em termos da dífêrença entre $\overline{y_{1}}$ e $\overline{y_{2}}$ relativo à variância dos $y^{\prime} s$. Isto é:

$$
\text { sep. }=\frac{\left|\overline{y_{1}}-\overline{y_{2}}\right|}{s_{y}}, \quad \text { onde } \quad s_{y}^{2}=\frac{\sum_{j=1}^{n_{1}}\left(y_{1 j}-\bar{y}_{1}\right)^{2}+\sum_{j=1}^{n_{2}}\left(y_{2 j}-\bar{y}_{2}\right)^{2}}{n_{1}+n_{2}-2} \text {. }
$$

O objetivo é selecionar a combinação linear de x para realizar a separação máxima das médias amostrais $\overline{y_{1}}$ e $\overline{y_{2}}$.

Resultado 2.4. : A combinação linear $y=\hat{\ell}^{\prime} x=\left(\bar{x}_{1}-\bar{x}_{2}\right)^{\prime} \mathrm{S}_{\text {pooded }}^{-1} x$ maximizu a razão:

$$
\begin{aligned}
& \frac{(S Q \operatorname{de} \bar{y})}{\operatorname{Var}(y)}=\frac{\left(\bar{y}_{1}-\bar{y}_{2}\right)^{2}}{s_{y}^{2}} \\
& =\frac{\left(\hat{\ell}^{\prime} \bar{x}_{1}-\hat{\ell}^{\prime} \hat{x}_{0}\right)^{2}}{\hat{\ell}^{\prime} \mathbf{s}_{\text {pooded }}} \\
& =\frac{(\boldsymbol{\ell} \ell d)^{2}}{\hat{\ell} \mathbf{S}_{\text {pooled }} \hat{\ell}}
\end{aligned}
$$

sobre todos os possiveis coeficientes $d \theta$ vetor $\hat{\ell}$, onde $d=\left(\bar{x}_{1}-\bar{x}_{2}\right)$ e $s_{y}^{2}$ também pode ser calculado camo:

$$
s_{y}^{2}=\frac{\sum_{j=1}^{n_{1}}\left(y_{1 j}-\bar{y}_{1}\right)^{2}+\sum_{j=1}^{n_{2}}\left(y_{2 j}-\bar{y}_{2}\right)^{2}}{n_{1}+n_{2}-2} .
$$


$\operatorname{com} y_{1 j}=\hat{\ell}_{x_{1 j}}$ e $y_{2 j}=\hat{\ell}_{x_{2 j}}$

O máximo da razão é dado por:

$$
\max _{\hat{\ell}} \frac{\left(\hat{\ell}^{\prime} \mathrm{d}\right)^{2}}{\hat{\ell}^{\prime} \mathbf{S}_{\text {pooled }} \hat{\ell}}=d^{\prime} \mathbf{S}_{\text {pooled }}^{-1} \mathrm{~d}=\left(\overline{\mathbf{x}}_{1}-\overline{\mathbf{x}}_{2}\right)^{\prime} \mathbf{S}_{\text {pooled }}^{-1}\left(\overline{\mathbf{x}}_{1}-\overline{\mathbf{x}}_{2}\right)=D^{2}
$$

onde $D^{2}$ é a distância quadrada amostral entre as duas médias.

A solução de Fisher para o problèma de separação pođe ser usado para classificar novas observaçōes.

Uma regra de alocação baseadà na Função Discriminante de Fisher :

Alocar $\mathrm{x}_{0}$ à $\pi_{1}$ se

$$
\begin{aligned}
& y_{0}=\left(\overline{\mathbf{x}}_{1}-\overline{\mathbf{x}}_{2}\right)^{\prime} \mathrm{S}_{\text {pooled }}^{-1} \mathrm{x}_{0} \geq \hat{m}=\frac{1}{2}\left(\overline{\mathbf{x}}_{1}-\overline{\mathbf{x}}_{2}\right)^{\prime} \mathrm{S}_{\text {pooded }}^{-1}\left(\overline{\mathbf{x}}_{1}+\overline{\mathbf{x}}_{2}\right) \text { ou } \\
& y_{0}-\hat{m} \geq 0 .
\end{aligned}
$$

caso contrário, alocar $\mathrm{x}_{0} \stackrel{\mathrm{a}}{\pi_{2}}$.

Perceba que esta regra de classificação é dàca em $(2.13)$ quando $\left(\frac{c(1 \mid 2)}{c(2 ! 1)}\right)\left(\frac{-p_{2}}{p_{1}}\right)=1$.

Em resumo, para duas populações, a separação máxima relativa que pode ser obtida considerando a combinação linear das observações multivariadas é igual à distancia $D^{2}$. Isto pode ser conveniente porque pode ser usadò em certas situações para testar se as médias populacionais $\mu_{1}$ e $\mu_{2}$ diferem significativamente. Conseqüentemente um teste para a difèrença nos vetores dè média pođè ser visto como um teste para a significância da separação a ser realizada.

Suponha que as médias populacionais $\mu_{1}$ e $\mu_{2}$ sejam médias das distribuições normais multivariadas com uma matriz de covariância comum $\boldsymbol{\Sigma}$. Um teste de $H_{0}: \boldsymbol{\mu}_{1}=\boldsymbol{\mu}_{2}$ contra $H_{0}: \mu_{1} \neq \mu_{2}$ é dado pelà estatística

$$
\left(\frac{n_{1}+n_{2}-p-1}{\left(n_{1}+n_{2}-2\right) p}\right) \cdot\left(\frac{n_{1} n_{2}}{n_{1}+n_{2}}\right) D^{2}
$$

que tern uma distribuição $F$ com $v_{1}=p$ e $v_{2}=n_{1}+n_{2}-p-1$ graus de liberdade. Se $H_{0}$ é rejeitada, podemos concluir que a separação entre as duas populaçōes $\pi_{1}$ e $\pi_{2}$ é significante.

\subsection{Classificação de Várias Populações}

Nesta seção apresentamos a generalização dos procedimentos de classificação para $g>2$ ou seja mais de 2 grupos. 


\subsubsection{Método do custo minimo de classificação errąda}

Seja $f_{i}(\dot{x})$ a densidade assaciada a poputaçãa $\pi_{i}$ para $i=1,2, \ldots, g$. (Em geral considera-se $f_{i}(\mathrm{x})$, densidadenormälmultivariada, mas essa não é uma condiçãa necessárịa)

Assim, sejam:

$p_{i}$ : probabilidade a priori da população $\pi_{i}$.

$c(k \mid i)$ : custo de alocar um item a $\pi_{k}$ quando de fato, ele pertence a $\pi_{i}$ para $k_{a} i=$ $\mathrm{t}, 2, \ldots, g$.

Para $k=i$, temos $c(i \mid i)=0$. Finalmente, , seja $R_{k}$ a conjunto dos $\mathrm{x}^{\prime} s$ classificados como $\pi_{k}$ então:

$P(k \mid i)=P\left(\right.$ classificar um item como $\left.\pi_{k} \mid \pi_{i}\right)=\int_{R_{k}} f_{i}(\mathrm{x}) d \mathrm{x}, \quad$ para $k, i=1,2, \ldots, g$. $\operatorname{com} P(i \mid i)=1-\sum_{k=1}^{g} P(k \mid i)$, para $k \neq i$.

$\mathrm{O}$ custo esperado condicional de uma classificação errada de um $\mathrm{x}$ de $\pi_{1}$, em $\pi_{2}$, ou $\pi_{3}, \ldots$, ou $\pi_{g}$ é:

$$
\operatorname{ECM}(1)=P(2 \mid 1) c(2 \mid 1)+P(3 \mid 1) c(3 \mid 1)+\ldots+P(g \mid 1) c(g \mid 1)=\sum_{k=2}^{g} P(k \mid 1) c(k \mid 1)
$$

Esse custo esperado condicional ocorre com probabilidade a priori $p_{1}$, a probabilidade de $\pi_{1}$.

Dè maneira similar calculamos $E C M(2), \ldots, E C M(g)$ - Portanto o custo esperado condicional geral de uma classificação errada é dado por:

$$
\begin{aligned}
E C M & =p_{1} \operatorname{ECM}(1)+p_{2} \operatorname{ECM}(2)+\ldots+p_{9} E C M(g) \\
& =\sum_{i=1}^{g} p_{i}\left(\sum_{k=1}^{g} P(k \mid i) c(k \mid i)\right), \text { para } k \neq i
\end{aligned}
$$

Resultado 2.5. : As regiões de classificação que minimizam o ECM são definidas por alocar $x$ a população $\pi_{k}, k=1, \ldots, g$ quạndo

$$
\sum_{i=1}^{g} p_{i} f_{i}(x) c(k / i), \quad \text { para } k \neq i
$$

seja o menor possivel.

Prova: ( ver Anderson, T.W, 1984).

Um caso especial é dado quando todos os $c(k \mid i)$ são iguais. Neste caso temos: 
Alocar x à população $\pi_{k}$ se

$$
p_{k} f_{k}(x)>p_{i} f_{i}(x), \quad \forall i \neq k
$$

ou equivalentemente,

Alócar x à população $\pi_{k}$ se

$$
\ln p_{k} f_{k}(x)>\ln p_{i} f_{i}(x), \quad \forall i \neq k
$$

Essa regra é idêntica a aquelà que maximiza a probabilidade a "posteriọri",

$$
P\left(\pi_{k} \mid \mathrm{x}\right)=\frac{p_{k} f_{k}(\mathrm{x})}{\sum_{i=1}^{g} p_{i} f_{i}(\mathrm{x})}=\frac{(\text { priori }) *(\text { veross })}{\sum[(\text { priori }) *(\text { veross })]^{2}} \quad \text { para } \quad k=1,2, \ldots, g
$$

\subsubsection{Classificação de Populações Normais}

Considerar $\mathrm{x}$ com densidade,

$$
f_{i}(\mathrm{x})=\frac{1}{(2 \pi)^{p / 2}\left|\Sigma_{i}\right|^{1 / 2}} \exp \left\{-\frac{1}{2}\left(\mathrm{x}-\mu_{i}\right)^{r} \Sigma_{i}^{-1}\left(\mathrm{x}-\mu_{i}\right)\right\} \quad i=1,2, \ldots, g .
$$

isto é, densidades normais multivariadas com vetor de média. $\mu_{i}$ e matriz dè covariância $\Sigma_{i-S e} c(i \mid i)=0$ e $c(k \mid i)=1$; para $k \neq i$ (custos iguaìs), então:

Alocar x à $\pi_{k}$ se

$$
\ln p_{k} f_{k}(\mathrm{x}) \doteq \operatorname{In} p_{k}-\left(\frac{p}{2}\right) \ln (2 \pi)-\frac{1}{2} \ln \left|\Sigma_{k}\right|-\frac{1}{2}\left(x-\mu_{k}\right)^{\prime} \Sigma_{k}^{1}\left(x-\mu_{k}\right)=\max _{i} \ln p_{i} f_{i}(x)
$$

A constante $\left(\frac{R}{2}\right) \operatorname{In}(2 \pi)$ pode ser descartada, daí podemos-definir o escore discriminạnte quadrático para a i-ésima população como,

$$
d_{i}^{Q}(\mathrm{x})=-\frac{1}{2} \ln \left|\Sigma_{i}\right|-\frac{1}{2}\left(\mathrm{x}-\mu_{i}\right)^{\prime} \mathbf{\Sigma}_{i}^{-1}\left(\mathrm{x}-\mu_{i}\right)+\ln p_{i}
$$

A regra que minimiza a probabilidadè total dè classificação errada para populàções normais quando temos $\boldsymbol{\Sigma}_{\boldsymbol{i}}$ diferentes, é dada por:

Alocar x à $\pi_{k}$ se

$$
d_{k}^{Q}(\mathrm{x})=\max \left(d_{1}^{Q}(\mathrm{x}), d_{2}^{Q}(\mathrm{x}), \ldots, d_{g}^{Q}(\mathrm{x})\right)
$$

$\mathrm{Na}$ prática $\boldsymbol{\mu}_{i}$ e $\boldsymbol{\Sigma}_{i}$ não são conhecidos, assim os substituímos respectivamente pelas quantidades amostrais:

$\overline{\mathbf{x}}_{i}$ : Vetor de média amostral

$\mathbf{S}_{i}$ : Matriz de covariância amostral 
onde $n_{i}$ é o tamanho amostral de cada população.

Neste caso, o escore discriminante quadrático é définido por:

$$
\tilde{d}_{i}^{Q}(x)=-\frac{1}{2} \ln \left|S_{i}\right|-\frac{1}{2}\left(x-\bar{x}_{i}\right)^{\prime} S_{i}^{-1}\left(x-\bar{x}_{i}\right)+\ln p_{i}
$$

e a regra de classificação baseada na amostra é dadà por :

Alocar $\mathrm{x}$ à $\pi_{k}$ se

$$
\hat{d}_{k}^{Q}(\mathrm{x})=\max \left(\hat{d}_{1}^{Q}(\mathrm{x}), \hat{d}_{2}^{Q}(\mathrm{x}), \ldots, \hat{d}_{g}^{Q}(\mathrm{x})\right)
$$

Se as matrizes de covariâncias $\mathbf{\Sigma}_{i}$ são iguais, o escore discriminante quadrátíco é dado por:

$$
d_{i}^{Q}(\mathrm{x})=-\frac{1}{2} \ln |\mathbf{\Sigma}|-\frac{1}{2}(\mathrm{x})^{\prime} \boldsymbol{\Sigma}^{-1} \mathrm{x}+\boldsymbol{\mu}_{i}^{\prime} \mathbf{\Sigma}^{-1} \mathrm{x}-\frac{1}{2} \boldsymbol{\mu}_{i}^{\prime} \boldsymbol{\Sigma}^{-1} \boldsymbol{\mu}_{i}+\ln p_{i}, \quad \text { para } \quad i=1,2, \ldots, g .
$$

Observar que os primeiros dois termos de $d_{i}^{Q}(\mathrm{x})$ são os mesmos para todo $i=1,2, \ldots, g$, portanto podem ser ignorados para propósitos de clàssificação. Assim o escore linear de classificação é dado por:

$$
d_{i}(\mathbf{x})=\mu_{i}^{\prime} \mathbf{\Sigma}^{-1} \mathbf{x}-\frac{1}{2} \mu_{i}^{\prime} \mathbf{\Sigma}^{-1} \mu_{i}+\operatorname{In} p_{i}
$$

Una estimativa $\hat{d}_{i}(\mathrm{x})$, para o escore diseriminante linear, baseado na estimativa "Spooled" da matriz de covariância é dado por:

$$
\hat{d}_{i}(x)=\bar{x}_{i}^{\prime} S_{p o o l e d}^{-1} x-\frac{1}{2} \bar{x}_{i}^{\prime} S_{p o o l e d}^{-1} \bar{x}_{i}+\ln p_{i}
$$

Assim a regra de classificação fica na forma:

Alocar x à $\pi_{k}$ se

$$
\hat{d}_{k}(\mathrm{x})=\max \left(\hat{d}_{1}(\mathrm{x}), \hat{d}_{2}(\mathrm{x}), \ldots, \hat{d}_{g}(\mathrm{x})\right)
$$

Podemos estimar a performance dessas regras de classificações amostrais, usando o procedimento de Lachenbruch(1968).

Se $n_{i M}^{H}$ é o número de observações classificadas incorretamente no i-ésimo grupo,para $i=1,2, \ldots, g$, então uma estimativa da taxa verdadeira de erro, $E(A E R)$ é dada por:

$$
\hat{E}(A E R)=\frac{\sum_{i=1}^{g} n_{i M}^{H}}{\sum_{i=1}^{g} n_{i}}
$$




\subsection{Alguns exemplos ilustrativos.}

Nesta seção apresentamos dois exemplos envolvendo o problema de classificação,Um exemplo é relacionado com Bioestatística (dados em Lee,1980, p. 349-351) e autro relacionado com Geologia (dados em Johnson \& Wichern,1982, p. 568-569). Em ambos os casos o objetivo é discriminar (indivíduos e regiōes) de acorđa com algumas variáveis.

Exemplo 2.1. Neste estudo temos 71 pacientes com Leucemia, dos quais 37 respodem a um tratamento e os outros 34 não respandem a esse tratamento (ver Tabela 2.3).Ou seja vamos ter duas populações $g=2$ e duas variáveis $p=2$. Nosso objetivo é discriminar indivíduos nesses dois grupos(respondem ou não respandem ao tratamenta) só com a informação das 2 váriaveis $x_{1}$ (idade) e $x_{2}(L I \%)$, onde (LI \%)é a taxa de medícamento ou dose.

$\mathrm{Na}$ Tabela 2.3, temos a tabela de dados.Nas figuras 2.1 e 2.2 temos histogramas de cada variável, para cada grupo, para termos idéia sobre a forma dos mesmos:

Tabela 2.3: Idade, LI\% e Resposta de 71 pacientes com leucemia

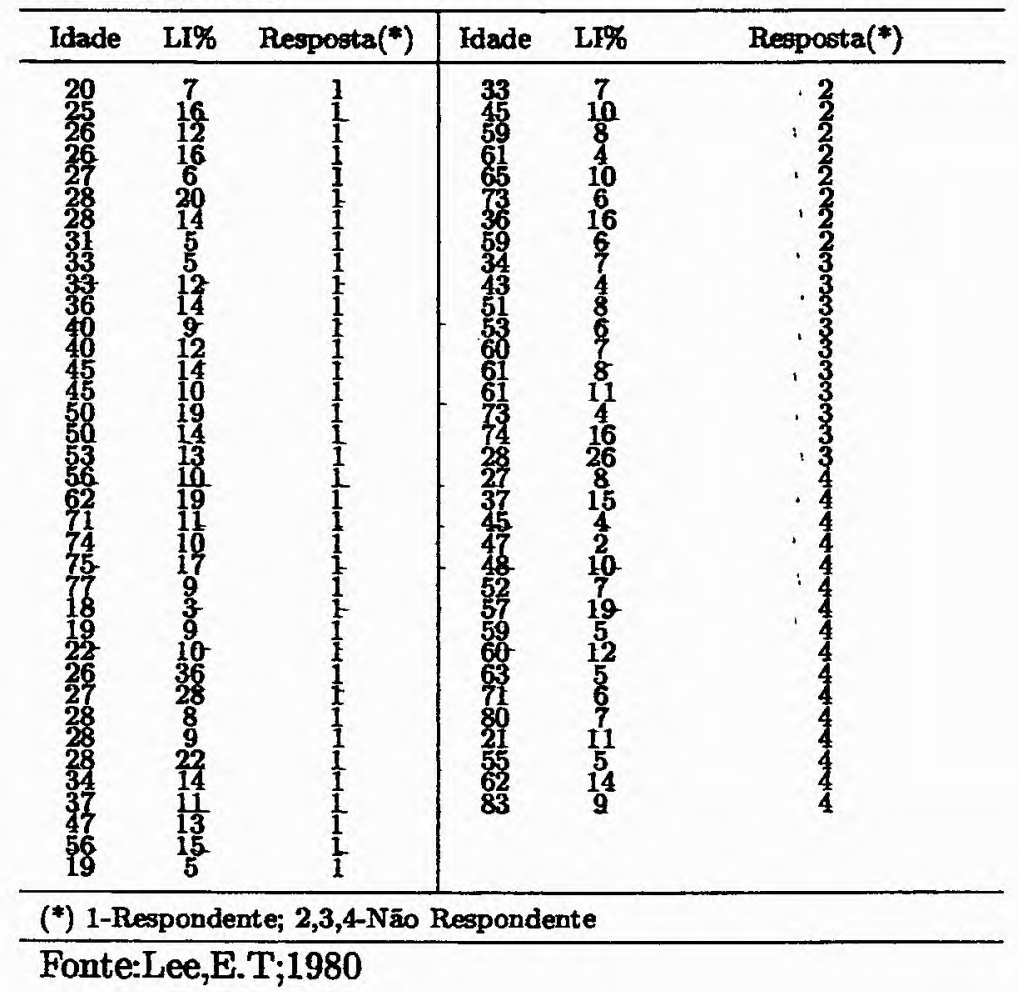



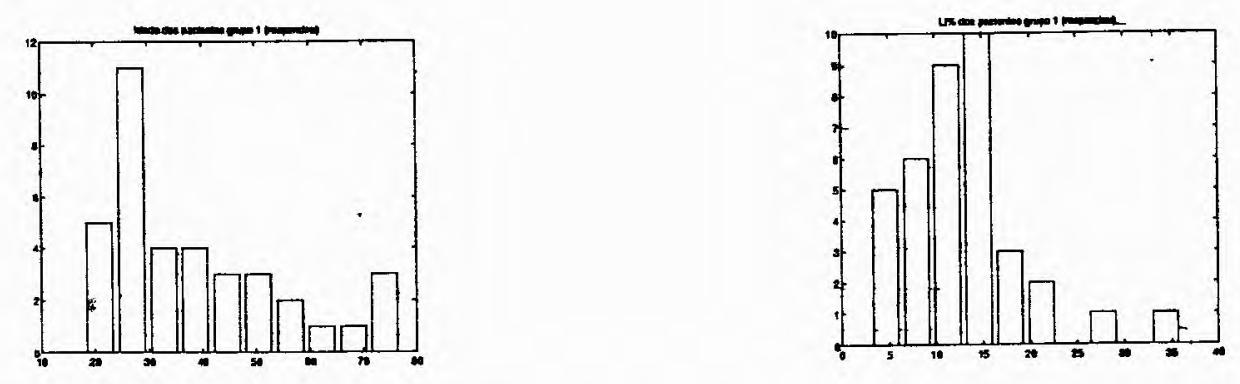

Figura 2.1: Histograma dos dados do grupo I (Respondente)
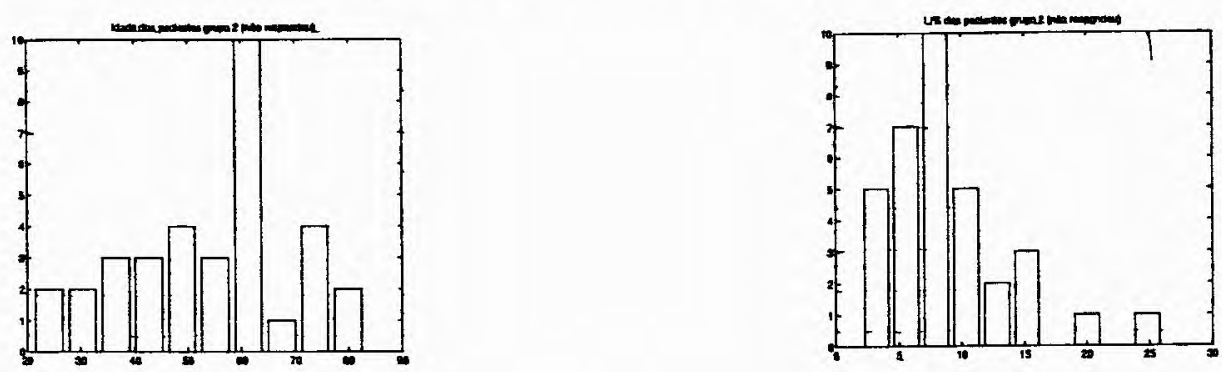

Figura 2.2: Histograma dos dados dă grupo 2 (Não-Respondente)

Neste exemplo consideramos $\left(\frac{c(1 \mid 2)}{c(2 \mid 1)}\right)\left(\frac{p_{2}}{p_{1}}\right)=1$, ou seja, os custos de classificação errados e as probabilidades a priori iguais para cada grupo.

Primeiramente, considerando $\Sigma_{i}=\Sigma, i=1,2$, vamos verificar se a separação entre as duas populações é significante ou seja testar se $H_{0}: \mu_{1}=\mu_{2}$ contra $H_{0}: \mu_{1} \neq \mu_{2}$.

Temos,

$$
n_{1}=37, \quad n_{2}=34, \quad p_{1}=2
$$

, assim

$$
\overline{\mathrm{x}}_{1}^{\ell}=\left(\begin{array}{ll}
38.9189 & 12.8919
\end{array}\right) ; \quad \mathrm{S}_{1}=\left(\begin{array}{cc}
287.0210 & 5.0465 \\
5.0465 & 41.9880
\end{array}\right)
$$

e

$$
\overrightarrow{\mathrm{x}}_{2}^{\prime}=\left(\begin{array}{ll}
54.0000 & 8.9118
\end{array}\right) ; \quad \mathrm{S}_{2}=\left(\begin{array}{cc}
237.2121 & -18.7576 \\
-18.7576 & 24.9920
\end{array}\right)
$$

Conseqüentemente,

$$
\mathbf{S}_{\text {pooled }}=\frac{\left(n_{1}-1\right) \mathbf{S}_{1}+\left(n_{2}-1\right) \mathbf{S}_{2}}{\left(n_{1}+n_{2}-2\right)}=\left(\begin{array}{cc}
263.1994 & -6.3380 \\
-6.3380 & 33.8595
\end{array}\right)
$$

e

$$
S_{\text {pooded }}^{-1}=\left(\begin{array}{cc}
0.0038 & 0.0007 \\
0.0007 & 0.0297
\end{array}\right)
$$


Logo

$$
D^{2}=\left(\overline{\mathbf{x}}_{1}-\overline{\mathbf{x}}_{2}\right)^{\prime} \mathbf{S}_{\text {pooled }}^{-1}\left(\overline{\mathbf{x}}_{1}-\overline{\mathbf{x}}_{2}\right)=1.2523
$$

e

$$
F=\left(\frac{n_{1}+n_{2}-p-I}{\left(n_{1}+n_{2}-2\right) p}\right)\left(\frac{n_{1} n_{2}}{n_{1}+n_{2}}\right) D^{2}=10.9331
$$

Ao nível de significância de $5 \%$ ternos $F_{p ;\left(n_{I}+n_{2}-p-1\right)}(0.05)=E_{2,68}(0.05)=3.131670$. Como $F>F_{2,68}(0.05)$ rejeitamos $H_{0}$, ou seja, existe uma diferença significativa entre $\mu_{1}$ e. $\mu_{2}$. Agora vamos utilizar as regras de classificação usuais, apoiados nos softwares "MìniTab 10.1 e MatLab 4.2".

Utilizando primeiro a Função Discriminante de Linear (F.D.L), ou seja, assumịndo a normalidade dos dados e matriz de covariância comum, temos à seguinte tabela de classificação:

Tabela 2.4:Tabela de Classificação

\begin{tabular}{c|c|cccc|c}
\multicolumn{7}{c}{ Pela Função Discriminante } \\
\hline & & $\pi_{1}$ & taxa & $\pi_{2}$ & taxa & valor real \\
\hline Membro & $\pi_{1}$ & 29 & 78.38 & 8 & 21.62 & 37 \\
Verdadeiro & $\pi_{2}$ & 8 & 23.53 & 26 & 76.47 & 34 \\
\hline
\end{tabular}

Assim a taxa de erro aparente $A P E R$ é igual a:

$$
A P E R=\frac{n_{1 M}+n_{2 M}}{n_{1}+n_{2}}=\frac{8+8}{37+34}=0.2253
$$

onde $n_{i M}, i=1,2$ é o número de classificações erradas para cada grupo ou seja, número de elemento que são do grupo $i$ e foram classificados no grupo $k$ onde $i, k=1,2 \mathrm{com}$ $i \neq k$.

Considerando $\boldsymbol{\Sigma}_{\boldsymbol{i}}$ diferentes, vamos ter a.Füção Discriminante Quadrática (F.D.Q), para este caso temos os seguintes resultados.

Tabela 2.5: Tabela de Classificação

Pela Função Discriminante

\begin{tabular}{c|c|cccc|c}
\hline & & $\pi_{1}$ & $\operatorname{taxa}$ & $\pi_{2}$ & taxa & valor real \\
\hline Membro & $\pi_{1}$ & 27 & 72.97 & 10 & 27.03 & 37 \\
Verdadeiro & $\pi_{2}$ & 8 & 23.53 & 26 & 76.47 & 34 \\
\hline
\end{tabular}


Assim a taxa de erro aparente $A P E R$ é igual a:

$$
A P E R=\frac{n_{1 M}+n_{2 M}}{n_{1}+\pi_{2}}=\frac{10+8}{37+34}=0.254
$$

Percebemos um desempenho relativamente bom, quando utilizamos a F.D.L e um. desempenho pior quando usamos a F.D.Q. Vamos verificar agora um exemplo com 3 populações e 5 variáveis.

Exemplo 2.2. Neste exemplo temos amostras de óleo cru de três zonas de arenito.

$$
\begin{aligned}
& \pi_{1}: \text { Wilhelm } \\
& \pi_{2}: \text { Sub-Mulinia } \\
& \pi_{3}: \text { Upper(Mulinia,segunda subescata,primeira subescqla) }
\end{aligned}
$$

Os valores de cada elemento são dados por,

$$
\begin{aligned}
& x_{1} \text { :vanádio, } \\
& x_{2} \text { :ferro, } \\
& x_{3} \text { :berilio, }
\end{aligned}
$$

\begin{tabular}{|c|c|c|c|c|}
\hline \multirow[b]{2}{*}{$x_{1}$} & \multirow[b]{2}{*}{$x_{2}$} & \multicolumn{3}{|c|}{$\operatorname{zana} x_{1}$} \\
\hline & & $x_{3}$ & $x_{4}$ & 20 \\
\hline $\begin{array}{l}3.9 \\
2.7 \\
2.8 \\
3.1 \\
3.9 \\
3.9\end{array}$ & $\begin{array}{l}51.0 \\
49.0 \\
36.0 \\
36.0 \\
46.0 \\
46.0 \\
45.0 \\
35.0\end{array}$ & $\begin{array}{l}0.29 \\
0.07 \\
0.30 \\
0.08 \\
0.10 \\
0.07 \\
0.00\end{array}$ & $\begin{array}{l}7.06 \\
7.11 \\
7.00 \\
7.20 \\
7.81 \\
6.25 \\
5.21 \\
\end{array}$ & $\begin{array}{l}12.19 \\
12.23 \\
11.30 \\
13.01 \\
12.63 \\
10.62 \\
.9 .00 \\
9.00\end{array}$ \\
\hline \multicolumn{5}{|c|}{ zona $\pi_{2}$} \\
\hline 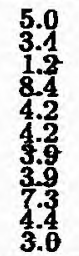 & 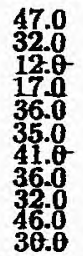 & 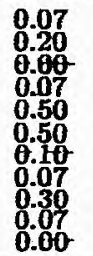 & $\begin{array}{l}7.06 \\
5.82 \\
56.51 \\
6.31 \\
9.25 \\
55.69 \\
56.19 \\
0.19 \\
7.02 \\
5.144\end{array}$ & 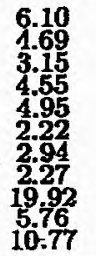 \\
\hline
\end{tabular}

e duas medidas para os hidrocarbonetos:

$$
\begin{aligned}
& x_{4}: \text { hidrocarbonetos saturados, } \\
& x_{5} \text { :hidrocarbonetos aromáticos, }
\end{aligned}
$$

Nosso objetivo é classificar cada observação com 5 variáveis em uma das 3 populações.

$\mathrm{Na}$ Tabela 2.6 temos os dados e nas figuras 2.1,2.2 e 2.3 os respectivos histogramas para cada variável em cada grupo. 
Continuação Tabela 2.5:

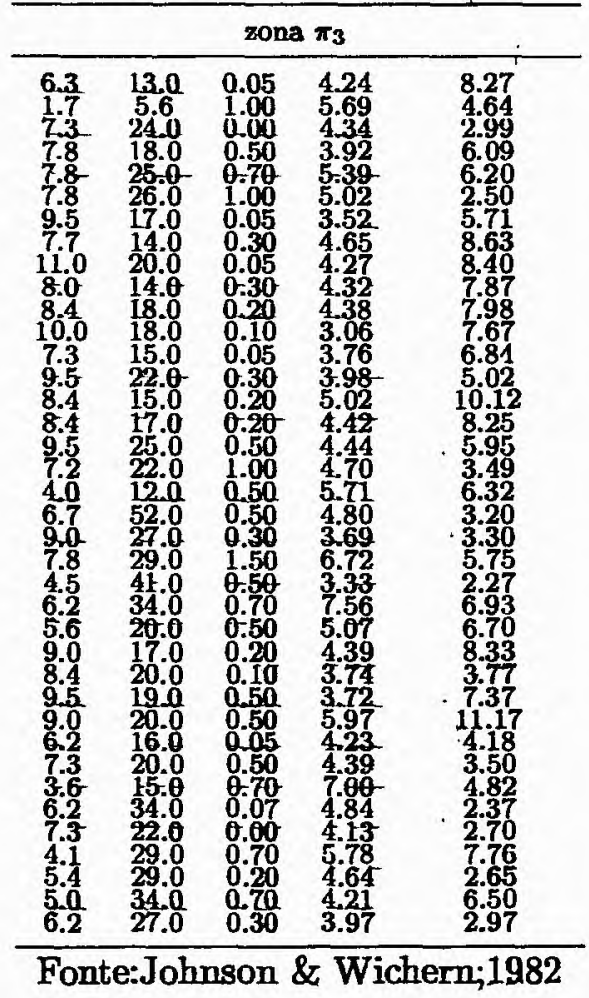
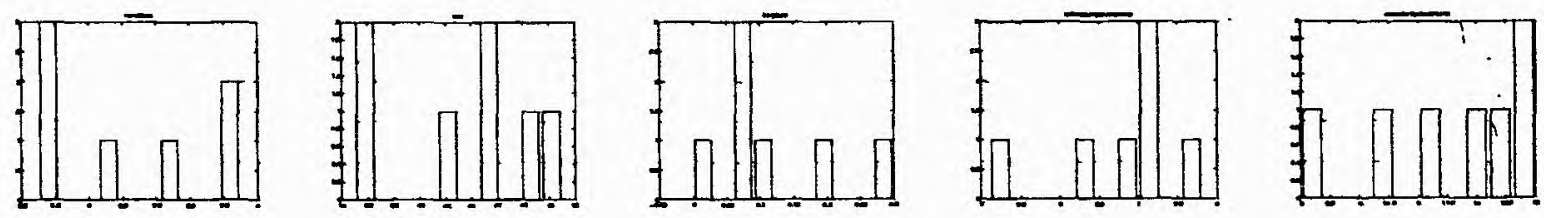

Figura 2.3: Histograma dos Dados da Primeira Zona de Arenito
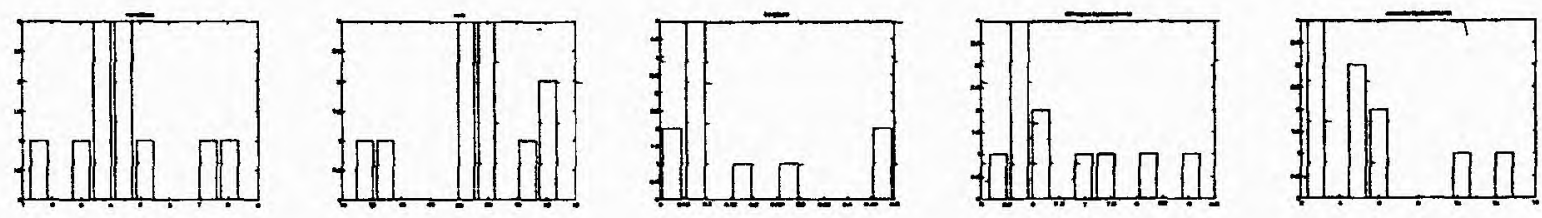

Figura 2.4: Histograma dos Dados da Segunda Zona de Arenito
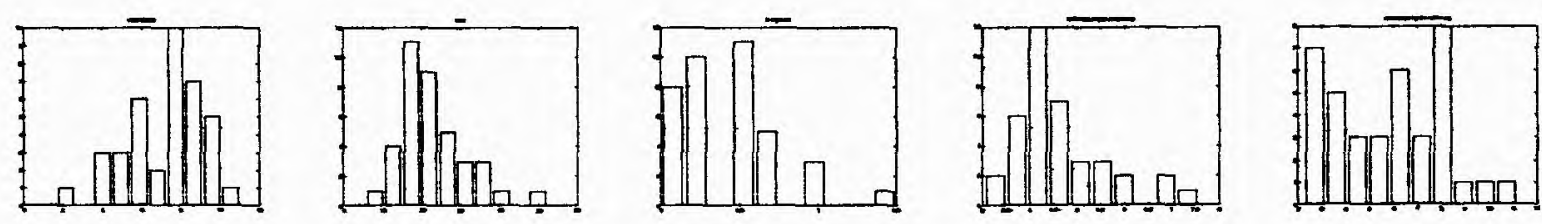

Figura 2.5: Histograma dos Dados da Terceira Zona de Arenito 
Para este exemplo, utilizamos as regras de classificação dadas na seção 2.5.2. Aqui, novamente consideramos $\left(\frac{c(1 \mid 2)}{c(2 \mid 1)}\right)\left(\frac{p_{2}}{p_{1}}\right)=1$. Os procedimentos são equivalentes a classificação pela Função Discriminante Linear (F.D.L) e Função Discriminante Quadrática (F.D.Q), quando trabalhamos com duas populações.

No primeiro caso, consideramos as matrizes de covariâncias $\boldsymbol{\Sigma}_{\boldsymbol{i}}$ iguais. Abaixo, apresentamos os seguintes resultados mostrados na Tabela 2.7 , tabela de classificação, e a estimativa do erro de classificação $A P E R$.

Tabela 2.7:Tabela de Classificação

Membro pelo Critério Discriminante

\begin{tabular}{l|c|cccccc|c}
\hline & & $\pi_{1}$ & taxa & $\pi_{2}$ & taxa & $\pi_{3}$ & taxa & valor real \\
\hline Membro & $\pi_{1}$ & 7 & 100.00 & 0 & 0.00 & 0 & 0.00 & 7 \\
& $\pi_{2}$ & 1 & 9.09 & 10 & 90.91 & $\theta$ & 0.00 & 11 \\
Verdadeiro & $\pi_{3}$ & $\theta$ & 0.00 & 3 & 7.89 & 35 & 92.11 & 38 \\
\hline
\end{tabular}

Assim,

$$
E C=\frac{n_{1 M}+n_{2 M}+n_{3 M}}{n_{1}+n_{2}+n_{3}}=\frac{\theta+1 亡 3}{7+11+38}=0.0714 .
$$

Quando consideramos $\boldsymbol{\Sigma}_{\boldsymbol{i}}$ diferentes, os resultados foram

Tabela 2.8:Tabela de Classificação

Membro pelo Critério Discriminante

\begin{tabular}{c|c|cccccc|c}
\hline & & $\pi_{1}$ & taxa & $\pi_{2}$ & taxa & $\pi_{3}$ & taxa & valor real \\
\hline Membro & $\pi_{1}$ & 7 & 100.00 & 0 & 0.00 & 0 & 0.00 & 7 \\
& $\pi_{2}$ & $\theta$ & 0.00 & 10 & 90.91 & 0 & 0.00 & 11 \\
Verdadeiro & $\pi_{3}$ & $\theta$ & 0.00 & 1 & 2.63 & 37 & 97.36 & 38 \\
\hline
\end{tabular}

Assim,

$$
A P E R=\frac{n_{1 M}+n_{2 M}+n_{3 M}}{n_{1}+n_{2}+n_{3}}=\frac{0+1+1}{7+11+38}=0.0357 .
$$

Pelas tabelas de classificação, percebemos uma considerável eficiência dos métodos, sendo que quando consideramos $\boldsymbol{\Sigma}_{\boldsymbol{i}}$ diferentes conseguimos um melhor desempenho.

Percebemos que o desempenho dos métodos de classificação, nos exemplos acjma, variam muito de caso para caso. No próximo capítulo, será mostrado o desempenho dessas regras de classificação, quando aplicamos diferentes metodologias de transformação de dados, baseados na transformação Box \& Cox. 


\section{Capítulo 3}

\section{Classificação e Discriminação com}

\section{Dados Transformados}

Na maioria dos exemplos utilizados para os problemas de classificação e discriminaçãa considera-se que o vetor $\mathbf{x}$ de observações tenha uma distribuição normal multivariada (ver por exempla Anderson,T.W,1984;Johnson,R.A \& Wichern,D.W,1982). No entąnto quando o vetor $\mathrm{x}$ não se aproxima muito-dessa distribuição, os procedimentos de classificação usuais podem não ser tão eficientes. Uma alternativa para o problema seria a transformaçãa dos dados para a forma normal(ver por exemplo Box\&Cax;1964).

Neste capítulo descrevemos o procedimento đe transformação de Box-Cox para o caso univariado e multivariado, apresentamos alternativas Bayesianas a partir dessa transformação, considerando as funçōes de verossimilhança perfilada e completa. Utilizamos os dados do exemplo visto no capítulo anterior, que foram transformados e depois efetuamos os procedimentos de classificaçãa. Os resultados foram comparados.

\subsection{Transformação dos dados para a forma Normal: Caso Univariado.}

Bax \& Cox(1964), introduz uma transformação que aproxima $x$ de uma distribuição normal dada por: 


$$
x^{(\lambda)}= \begin{cases}\frac{x^{\lambda}-1}{\lambda} & \text { se } \lambda \neq 0 \\ \ln (x) & \text { se } \lambda=0 .\end{cases}
$$

Na prática podemos ter dificuldade para encontrar um valor de $\lambda$ que viabilize tal transformação. O procedimento usado é assumir $y=x^{(\lambda)} \sim N\left(\mu, \sigma^{2}\right)$. Asssim

$$
f(y)=\frac{1}{\sqrt{2 \pi} \sigma} \exp \left\{-\frac{1}{2 \sigma^{2}}(y-\mu)^{2}\right\}
$$

isto é,

$$
f\left(x^{\lambda}\right)=\frac{1}{\sqrt{2 \pi} \sigma} \exp \left\{-\frac{1}{2 \sigma^{2}}\left(x^{(\lambda)}-\mu\right)^{2}\right\}
$$

De (3.1), temos

$$
y=\frac{x^{\lambda}-1}{\lambda}, \text { isto é, } x^{\lambda}=\lambda y+1
$$

Portanto,

$$
x=(\lambda y+1)^{\frac{1}{\lambda}} \text { e } \frac{d x}{d y}=\frac{1}{\lambda}(\lambda y+1)^{\frac{1}{\lambda}-1} \lambda
$$

Assim

$$
\frac{d x}{d y}=\left(x^{\lambda}\right)^{\frac{1}{\lambda}-1}, \text { isto é, }\left|\frac{d x}{d y}\right|=x^{1-\lambda}, \text { ou }\left|\frac{d y}{d x}\right|=x^{x+1}
$$

Portanto,

$$
f(x)=\frac{1}{\sqrt{2 \pi} \sigma} \exp \left\{-\frac{1}{2 \sigma^{2}}\left(x^{(\lambda)}-\mu\right)^{2}\right\} x^{\lambda-1}
$$

\subsubsection{Função de Verossimilhança para- $\lambda, \mu$ e $\sigma^{2}$}

Seja $x_{1}, x_{2}, \ldots, x_{n}$ uma amostra aleatória de tamanho $n$. A função de verossimilhança para $\lambda, \mu$ e $\sigma^{2}$ é dada por:

$$
L\left(\lambda, \mu, \sigma^{2}\right)=\left(2 \pi \sigma^{2}\right)^{-\frac{n}{2}} \exp \left\{-\frac{1}{2 \sigma^{2}} \sum_{i=1}^{n}\left(x_{i}^{(\lambda)}-\mu\right)^{2}\right\} \prod_{i=1}^{n} x_{i}^{\lambda-1}
$$

O lógaritmo da verossimilhañça é dado por;

$$
l\left(\lambda, \mu, \sigma^{2}\right)=-\frac{n}{2} \ln \left(\sigma^{2}\right)-\frac{1}{2 \sigma^{2}} \sum_{i=1}^{n}\left(x_{i}^{(\lambda)}-\mu\right)^{2}+(\lambda-1) \ln \left(\sum_{i=1}^{n} x_{i}\right)
$$


Fixada $\lambda$, os E.M.V's de $\mu$ e $\sigma^{2}$ são encontrados pelas equaçôes

$$
\frac{\partial l}{\partial \mu}=-\frac{1}{\sigma^{2}} \sum_{i=1}^{n}\left(x_{i}^{(\lambda)}-\mu\right)=0
$$

e

$$
\frac{\partial l}{\partial \sigma^{2}}=-\frac{n}{2 \sigma^{2}}+\frac{1}{2\left(\sigma^{2}\right)^{2}} \sum_{i=1}^{n}\left(x_{i}^{(\lambda)}-\mu\right)^{2}=0
$$

de onde vamos ter,

$$
\hat{\mu}=\frac{\sum_{i=1}^{n} x_{i}^{(\lambda)}}{n}=\overline{x^{(\lambda)}}
$$

e

$$
\tilde{\sigma^{2}}=\frac{1}{n} \sum_{i=1}^{n}\left(x_{i}^{(\lambda)}-\overline{x^{(\lambda)}}\right)^{2}
$$

Nosso objetivo agora é determinạr $\hat{\lambda}$.

\subsubsection{Uso do logaritmo da função de verossimilhança perfitada ("Profile Log-Likelihood").}

Substituindo os estimadores encontrados, (3.7) e (3.8) na expressāo (3.6), chegamos numa expressão, a menos de constantes, que envolve somente $\lambda$ e os dados, dada por:

$$
l(\lambda)=-\frac{\pi}{2} \ln \left[\frac{1}{n} \sum_{i}^{n}\left(x_{i}^{\lambda}-\overline{x^{\lambda}}\right)^{2}\right]+(\lambda-1) \sum_{i=1}^{n} \ln x_{i}
$$

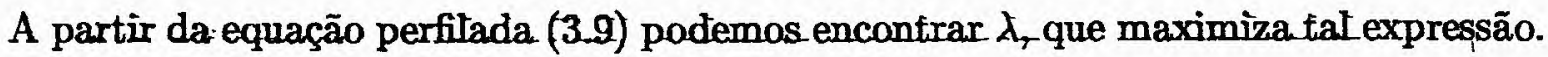
Primeiro atribuímos um intervalo grande para $\lambda$ a qual suspeitamos conter o ponto de máximo e através de várias tentativas, selecionamos o mais indicado. Identificado o intervalo, particionamos o mesmo de acordo com a precisão requerida e testamos qada ponto substituindo na expressão acima, até encontrar um ponto tal que maximize-a. Encontrado o valor de $\lambda$, realizar a transformação de acordo com

$$
x^{(\lambda)}= \begin{cases}\frac{x^{\lambda}-1}{\lambda} & \text { se } \lambda \neq 0, \\ \ln (x) & \text { se } \lambda=0 .\end{cases}
$$

Encontrar tal estimativa com uma boa precisão, requer um grande número de operações que só se viabiliza com a utilização de recursos computacionais. Esses programas foram desenvolvidos usando o software Matarab 4.2. 


\subsection{Transformação de Dados Multivariados (TBox).}

Seja $\mathrm{x}$ um vetor aleatório $(p \times 1)$. Para efetuarmos a procedimento de transformação de Box-Cox, temos que encontrar os valores $\hat{\lambda}_{1}, \hat{\lambda_{2}}, \ldots, \hat{\lambda_{p}}$ que maximizam conjuntamente a expressão:

$l\left(\lambda_{1}, \lambda_{2}, \ldots, \lambda_{p}\right)=-\frac{n}{2} \ln |S(\lambda)|+\left(\lambda_{1}-1\right) \sum_{j=1}^{n} \ln x_{1 j}+\left(\lambda_{2}-1\right) \sum_{j=1}^{n} \ln x_{2 j}+\ldots+\left(\lambda_{p}-1\right) \sum_{j=1}^{n} \ln x_{p j}$

que é a generalização de (3.9), onde $S(\lambda)$ é a matriz de covariância calculada a partir da matriz de dados transformados

$$
\mathrm{x}_{j}^{\lambda}=\left(\begin{array}{c}
\frac{x_{1 j}^{\lambda_{1}}-1}{\lambda_{1}} \\
\frac{x_{2 j}^{\lambda_{2}}-1}{\lambda_{2}} \\
\\
\frac{x_{p j}^{\lambda_{p}-1}}{\lambda_{p}}
\end{array}\right) ; \quad j=1,2, \ldots, n .
$$

Este procedimento é muito trabalhoso e requer um grande esforço computacional, Se $p>3$ o problema fica bastante complicado.

Para simplificação, selecionamos uma transformação Box-Cox para cada variável como no caso univariado e daí estimamos os $\lambda^{\prime} s$ para aplicar a transformação.

Sejam $\lambda_{1}, \lambda_{2}, \ldots, \lambda_{p}$ os parâmetros para cada transformação, cada $\lambda_{k}$ pode ser selecionado maximizando,

$$
l_{k}(\lambda)=-\frac{n}{2} \ln \left[\frac{1}{n} \sum_{j=1}^{n}\left(x_{k j}^{\left(\lambda_{k}\right)}-\overline{x_{k}^{\lambda_{k}}}\right)^{2}\right]+\left(\lambda_{k}-1\right) \sum_{i=1}^{n} \ln x_{k j}
$$

para $k=1,2, \ldots, p ; \lambda=\left(\lambda_{1}, \ldots, \lambda_{p}\right)$.

Com $x_{k 1}, x_{k 2}, \ldots, x_{k n}, n$ observaçōes na $k$-ésima variável, temos,

$$
\overline{x_{k}^{\lambda_{k}}}=\frac{1}{n} \sum_{j=1}^{n} x_{k j}^{\left(\lambda_{k}\right)}=\frac{1}{n} \sum_{j=1}^{n}\left(\frac{x_{k j}^{\lambda_{k}}-1}{\lambda_{k}}\right)
$$


Assim, a $j$-ésima observação transformada é dada por,

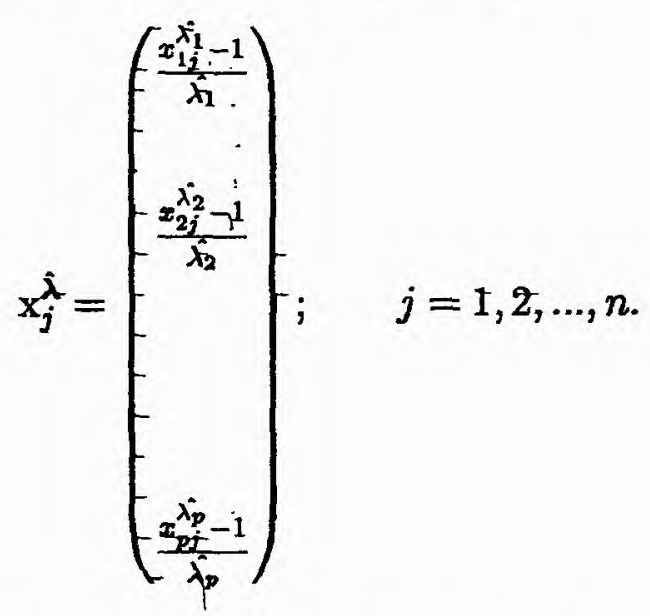

onde $\hat{\lambda}=\left(\hat{\lambda_{1}}, \hat{\lambda_{2}}, \ldots, \hat{\lambda_{p}}\right)$, maximiza individualmente $(3: 11)$.

Este procedimento é equivalente a levar cada distribuição marginal próxima da distribuição Normal. Isso não garante a normalidade multivariada, mas na prática pode melhorar muito a aproximação para a distribuição Normal Multivariada.

Uma alternativa para o problema, seria a utilização de procedimentos Bayesianos e a uso de MCMC para gerar amostras dos parâmetros $\lambda_{1}, \lambda_{2}, \ldots, \lambda_{p}$. Daí, encontramos $\hat{\lambda_{1}}, \hat{\lambda_{2}} \ldots, \hat{\lambda_{p}}$ que maximizam conjuntamente a expressāo (3.10).

\subsection{Enfoque Bayesiano para a transformação Box- Cox (TBoxi).}

Dada a dificutdade que envolve a transformação de $x_{p \times 1}$,um vetor ałeatório de dạdos multivariados para a forma normal multivariada, o uso dos procedimentos Bayesianos, considerando, distribuições a priori apropriadas para os parâmetros $\lambda_{1}, \lambda_{2}, \ldots, \lambda_{p}$, conjuñ tamente com o uso de métodos MCMC(Monte Carlo em Cadeias de Markov), se mostrou uma boa alternativa para o problema da transformação.

\subsubsection{Distribuições a priori para os parâmetros $\lambda_{1}, \lambda_{2}, \ldots, \lambda_{p}$}

Consideramos distribuições a priori informativas para os parâmetros $\lambda_{1}, \lambda_{2}, \ldots, \lambda_{p}$. Assim, vamos propor distribuições Normais, como por exemplo, $N\left(a_{k}, b_{k}^{2}\right)$, onde $a_{k}$ e $b_{k}^{2}$ são conhecidos e variam para cada $\lambda_{k}, k=1, \ldots, p$. 
Como não temos um algoritmo eficiente para a estimação da moda, a escolha de distribuições a priori normais facilita tal estimação.

\subsubsection{Distribuição a posteriori conjunta para os parâmetros}

A. funçãa de verossimilhança perfilada para $\lambda_{1}, \lambda_{2}, \ldots, \lambda_{2}$ é dada por,

$$
L\left(\lambda_{1}, \ldots, \lambda_{p}\right)=\exp \left\{-\frac{n}{2} \ln |S(\lambda)|+\sum_{k=1}^{p} \sum_{j=1}^{n}\left(\lambda_{k}-1\right) \ln \left(x_{k j}\right),\right\}
$$

Assumindo as distribuições a priori Normais independentes, a distribuição a priori conjunta para $\lambda_{1}, \ldots, \lambda_{p}$ é dada por,

$$
\pi\left(\lambda_{1}, \lambda_{2}, \ldots, \lambda_{p}\right)=\prod_{k=1}^{p} N\left(a_{k}, b_{k}^{2}\right)
$$

Portanto a distribuição a posteriori conjunta pode ser escrita como

$$
\begin{aligned}
\pi\left(\lambda_{1}, \lambda_{2}, \ldots, \lambda_{p} \mid x_{1}^{\left(\lambda_{1}\right)}, x_{2}^{\left(\lambda_{2}\right)}, \ldots, x_{p}^{\left(\lambda_{p}\right)}\right) & \propto \pi\left(\lambda_{1}, \lambda_{2}, \ldots, \lambda_{p}\right) E\left(\lambda_{1}, \ldots, \lambda_{p}\right) \\
& \propto\left(\prod_{k=1}^{p} N\left(a_{k}, b_{k}^{2}\right)\right) \exp \left\{-\frac{n}{2} \ln |S(\lambda)|+\sum_{k=1}^{p} \sum_{j=1}^{n} \lambda_{k} \ln \left(x_{k j}\right)\right\},
\end{aligned}
$$

\subsubsection{Distribuições . Condicionais a Posteriori necessárias para o algoritmo Gibbs Sampling.}

Da expressão (3.13), vamos derivar as distribuiçōes condicionais, necessárias para a gerar amostras via simulação MCMC para análise Bayesiana.

Pàra cada parâmetro $\lambda_{k},(k=I, \ldots, p)$ a distribuiçãò condicional tem a fợina

$$
\pi\left(\lambda_{k} \mid \lambda_{k^{\prime}}, \mathrm{x}^{(\lambda)}\right) \propto N\left(a_{k}, b_{k}^{2}\right) \exp \left\{-\frac{n}{2}|S(\lambda)|+\lambda_{k} \sum_{j=1}^{n} \ln x_{k j}\right\}
$$

onde $\lambda_{k^{\prime}}=\left(\lambda_{1}, \ldots, \lambda_{k-1}, \lambda_{k+1}, \ldots, \lambda_{p}\right)$

Neste caso vamos utilizar o algoritmo Metropolis-Hastings, onde os núcleos para a geraçãō dos candidatos $\lambda_{k}$ 's sãà as dis̄tribuiçães $N\left(\check{a}_{k}, b_{k}^{2}\right)$ 's.e as probabilidàdes de acei- 
tação são calculàdäs por

$$
\min \left\{\frac{\Psi_{k}\left(\lambda^{(s+1)}, x^{\lambda}\right)}{\Psi_{k}\left(\lambda^{(s)}, x^{\lambda}\right)}, 1\right\}
$$

onde $s+1$ indica a iteração atual e $s$ a iteração anterior e,

$$
\Psi_{k}(\cdot)=\exp \left\{-\frac{n}{2} \ln \mid S(\lambda) H+\lambda_{k} \cdot \sum_{j=1}^{n} \ln \left(x_{k j}\right)\right\} .
$$

Gerada è seléciònada a ämostra dē parâmetros $\lambda_{\bar{k}}$ para $k=1, . ., p$, calcūulāmos para cada conjuntō a moda amostral que ế uma estimativa dos $\lambda_{k}$ que maximiżàn (3.10) conjuntamente.

\section{4- Outro Enfoque Bayesiano: O uso dä Função de Vèrossimilhança Completa (TBox2).}

Na seção anterior, utilizamos o logaritmo da função de verossimilhança perfilada para encontrar as estimativas dos parâmetros de tränsfórmação.

Dentro do enfoque Bayesiano, a utilização da verossimilhança perfilada pode ser perígoso devidö a forte influência da distribuição a priori no modelo.

Nesta seção tentamos uma alternativa para este problema, utilizando a função de verossimilhănça completa e disstribūiçōè a priòi apropriadas.

\subsection{1: Estimação dos parâmetros de transformação: caso uni- variädo.}

Dado $x_{1}, x_{2}, \ldots, x_{n}$ uma amostra aleatória de tamanho $n$, a função de verossimilhança completa para o caso univariàdò foì đadā em (3.5) cöno,

$$
L\left(\lambda, \mu, \sigma^{2}\right)=\left(2 \pi \sigma^{2}\right)^{-\frac{n}{2}} \exp \left\{-\frac{1}{2 \sigma^{2}} \sum_{i=1}^{n}\left(x_{i}^{(\lambda)}-\mu\right)^{2}\right\} \prod_{i=1}^{n} x_{i}^{\bar{\lambda}-1}
$$

Vamos consì̃ērar distribuiçōes a priori para $\lambda, \mu$ e $\sigma^{2}$ đädās pòr,

$$
\begin{aligned}
& \mu \sim N\left(\mu_{0}, \alpha_{0}^{2}\right)_{\gamma} \text { ondē } \mu_{0} e \sigma_{0}^{2} \text { sãöconhëcidos. } \\
& \sigma^{2} \sim \text { IG }\left(a ; \frac{1}{b}\right) \text {, ondè } a \text { e } b \text { são confiecidos. } \\
& \lambda \sim N\left(c, d^{2}\right) \text {; ondec ce } d \text { - são conheciđos. }
\end{aligned}
$$




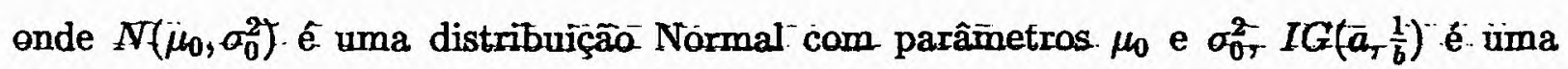
distribuição Gama Invertida com parâmetros $a$ e $\frac{1}{b}$, e $N\left(c_{,} d^{2}\right)$ também é uma distribüição Normal com parâmetros $c \mathrm{e}^{-d^{2}}$.

Assumindo que as distribuições a priori sejam independentes, a distribuição conjunta para $\lambda, \mu$ e. $\sigma^{2}$ é dàda por,

$$
\pi\left(\lambda, \mu, \sigma^{2}\right) \propto \exp \left\{\frac{I}{2 \sigma_{0}^{2}}\left(\mu-\mu_{0}\right)^{2}\right\}\left(\sigma^{2}\right)^{-(a+1)} \exp \left\{-\frac{1}{\sigma^{2} b}\right\} N\left(c, d^{2}\right)
$$

Portanto a distribuição a posteriori conjunta é dada por,

$$
\begin{aligned}
& \pi\left(\lambda, \mu, \sigma^{2} \mid D\right) \propto\left(\sigma^{2}\right)^{-\frac{n}{2}} \exp \left\{-\frac{1}{2 \sigma^{2}} \sum_{i=1}^{n}\left(x_{i}^{(\lambda)}-\mu\right)^{2}\right\}\left[\prod_{i=1}^{n} x_{i}^{\lambda-1}\right] \exp \left\{\frac{1}{2 \sigma_{0}^{2}}\left(\mu-\mu_{0}\right)^{2}\right\} \\
& \left(\sigma^{2}\right)^{-(a+1)} \exp \left\{-\frac{1}{\sigma^{2} b}\right\} N\left(c, \dot{d}^{2}\right)
\end{aligned}
$$

onde $D . \ddot{=}\left\{\bar{x}_{1}, \ldots, x_{i n}\right\}$.

Aš̀-distribüições condicioñais a posteriori necessárias para o algoritino Gỉbbs Sampạling para cada parâmetro são dadas abaixo.

Pàra $\sigma^{2}$ tèmos,

$$
\pi\left(\sigma^{2} \mid \mu,-\lambda ; D\right) \propto\left(\sigma^{2}\right)^{-\left(\frac{n}{2} \pm a+1\right)} \exp \left\{\frac{1}{-\sigma^{2}\left(\frac{1}{6} \pm \frac{1}{2} \sum_{i=1}^{n}\left(x_{i}^{(\lambda)}-\mu\right)^{2}\right)^{-1}}\right\}
$$

Portanto,

$$
\left(\sigma^{2} \mid \mu, \lambda, D\right) \sim F G\left(\frac{n}{2}+a,\left(\frac{1}{b}+\frac{1}{2} \sum_{i=1}^{n}\left(x_{i}^{(\lambda)}-\mu\right)^{2}\right)^{-1}\right)
$$

Para $\mu$ vamos ter,

$$
\pi\left(\mu \mid \sigma^{2}, \lambda, D\right) \propto\left[\prod_{i=1}^{n} \exp \left\{-\frac{1}{2 \bar{\sigma}^{2}}\left(x_{i}^{(\lambda)}=\mu\right)^{2}\right\} \exp \left\{-\frac{1}{2 \sigma_{0}^{2}}\left(\mu-\mu_{0}\right)^{2}\right\} .\right.
$$

Desenvolvendo esta expressão, temos,

$$
\left(\mu \mid \sigma^{2} ; \lambda ; D\right) \sim \AA\left(b_{1}, \overrightarrow{a_{T}^{-}}\right) \ldots
$$


onde

$$
b_{1}=\frac{\frac{\mu_{0}}{\sigma_{0}^{2}}+\frac{\overline{n x_{i}^{(\lambda)}}}{\sigma^{2}}}{\frac{1}{\sigma_{0}^{2}}+\frac{n}{\sigma^{2}}} \quad \text { e } \quad a_{1}^{-1-}=\frac{1}{\frac{1}{\sigma_{Q}^{2}}+\frac{n}{\sigma^{2}}} \text {. }
$$

Para $\lambda$ vamos ter,

$$
\pi\left(\lambda \mid \mu, \sigma^{2} ; D\right) \propto N\left(c, d^{2}\right) \Psi_{1}(\cdot)
$$

onde

$$
\begin{aligned}
\Psi_{1}(.) & =\exp \left\{-\frac{1}{2 \sigma^{2}} \sum_{i=1}^{n}\left(x_{i}^{\lambda}-\mu\right)^{2}\right\} \prod_{i=1}^{n} x_{i}^{\lambda-1} \\
& =\exp \left\{-\frac{1}{2 \sigma^{2}} \sum_{i=1}^{n}\left(x_{i}^{\lambda}-\mu\right)^{2}+(\lambda-1) \sum_{i=1}^{n} \ln \left(x_{i}\right)\right\}
\end{aligned}
$$

Portanto neste caso utilizamos o algoritmo Gibbs Sampling para a geração das amostras dos parâmetros de $\sigma^{2}$ e $\mu$, juntamente.com a algoritmo Metropolis-Hastingns para a geração dà amostra do parâmetro $\lambda$, ondè ò núcleo dè geraçãâ é uma distribuiçãō $N\left(c_{,} d^{2}\right)$; com o valor $\Psi_{1}($ ) $)$ usadò no cálculo de aceitạção.

\subsubsection{Estimação dỏs parâmetros de transformação: caso multi- variàdō.}

Para o caso multivariado temos,

$$
y_{i}=x_{i}^{(\lambda)}=\left(x_{i 1}^{\left(\lambda_{i} I\right.} ; \ldots, x_{i p}^{\left(\lambda_{p}\right)}\right)
$$

no qual $y_{i}$ é uno vetor aleatốrio ìidna forma $N_{p}(\dot{\mu}, \Sigma) ; i=1 ;, ;, n$.

Or seja,

$$
\left.f\left(x_{i}^{(\lambda)}\right] \mu, \Sigma\right)=(2 \pi)^{-\frac{2}{2}}[\Sigma]^{-\frac{1}{2}} \exp \left\{-\frac{1}{2}\left(x_{i}^{(\lambda)}-\mu\right)^{\prime} \Sigma^{-1}\left(x_{i}^{(\lambda)}-\mu\right)\right\}
$$

Aqui,

$$
x_{i k}^{\left(\lambda_{k}\right)}= \begin{cases}\frac{x_{i k}^{\lambda_{k}}-1}{\lambda_{k}} & \text { se } \lambda_{k} \neq 0 \\ \ln \left(x_{i k}\right) & \text { se } \lambda_{k}=0\end{cases}
$$

para $k=1, \ldots, p$.

Fazendo a transformação temos, 


$$
f\left(\mathbf{x}_{i} \mid \boldsymbol{\lambda}, \boldsymbol{\mu}, \boldsymbol{\Sigma}\right) \propto|\boldsymbol{\Sigma}|_{-}^{-\frac{1}{2}} \exp \left\{-\frac{1}{2}\left(\dot{x}_{i}^{(\lambda)}-\mu\right)^{\prime} \Sigma^{-1}\left(\bar{x}_{i}^{(\lambda)}-\mu\right) \xi \mid J[\right.
$$

onde

com

$$
J=\left(\begin{array}{cccc}
\frac{\partial y_{1}}{\partial x_{1}} & \frac{\partial y_{1}}{\partial x_{2}} & \cdots & \frac{\partial y_{1}}{\partial x_{p}} \\
\frac{\partial y_{2}}{\partial x_{1}} & \frac{\partial y_{2}}{\partial x_{2}} & \cdots & \frac{\partial y_{p}}{\partial x_{p}} \\
\vdots & \vdots & \ddots & \vdots \\
\frac{\partial y_{p}}{\partial x_{1}} & \frac{\partial y_{p}}{\partial x_{2}} & \cdots & \frac{\partial y_{p}}{\partial x_{p}}
\end{array}\right)
$$

$$
y_{k}=x_{k}^{\left(\lambda_{k}\right)}= \begin{cases}\frac{x_{k}^{x_{k}}-1}{\lambda_{k}} & \text { se } \lambda_{k} \neq 0, \\ \ln \left(x_{k}\right) & \text { se } \lambda_{k}=0\end{cases}
$$

para $k=1, \ldots, p$.

Daí,

$$
|J|=\left|\begin{array}{cccc}
x_{1}^{\lambda_{1}-1} & 0 & \cdots & 0 \\
0 & x_{2}^{\lambda_{2}-1} & \cdots & 0 \\
\vdots & \vdots & \ddots & \vdots \\
0 & 0 & \cdots & x_{p}^{\lambda_{p}-1}
\end{array}\right|=\prod_{k=1}^{p} x_{k}^{\lambda_{k}-1}
$$

Com $n$ observações $\mathrm{x}_{i}$ independentes temos,

$$
\left.L(\lambda, \mu, \Sigma) \propto f \Sigma\right|^{-\frac{n}{2}} \exp \left\{-\frac{1}{2} \sum_{i=1}^{n}\left(x_{i}^{(\lambda)}-\mu\right)^{\prime} \Sigma^{-1}\left(x_{i}^{(\lambda)}-\mu\right)\right\} \prod_{i=1}^{n} \prod_{k=1}^{p} x_{i k}^{\lambda_{k}-1}
$$

Vamos considerar as distribüições a priōi para $\boldsymbol{\mu}$ e $\mathbf{\Sigma}$.propostas por Prèss,S.J(1972) e.Box \& Tiàn(1976), que são.dadàs por,

$$
\begin{aligned}
& \pi(\mu) \propto \text { constānte } \\
& \pi(\mathbf{\Sigma}) \propto-|\Sigma|=\frac{1}{2}(p+1) \\
& \pi(\lambda) \propto \prod_{k=1}^{p-} N\left(a_{k}, b_{k}\right), \text { coma } a_{k}, b_{k}^{-\cdots} \text { conhecidos. }
\end{aligned}
$$

Asssumindo que as distribüuições a priori sejañ indēpendentes, a distribuição a postèriori conjunta é dada por,

$$
\pi(\mu, \Sigma, \lambda \mid D) \propto|\Sigma|^{-\frac{1}{2}(n+p+1)} \exp \left\{-\frac{1}{2} \sum_{i=1}^{n}\left(x_{i}^{\lambda}-\mu\right)^{\prime} \Sigma^{-1}\left(x_{i}^{\lambda}-\mu\right)\right\} \prod_{i=1-k=1}^{n} \prod_{i k}^{p} x_{k}^{\lambda_{k}-1}
$$


Isto é,

$$
\begin{aligned}
& \pi(\mu, \Sigma, \lambda \mid D) \propto|\Sigma|^{-\frac{1}{2}} \exp \left\{-\frac{n}{2}\left\{f\left(\mu-\overline{x^{(\lambda)}}\right)^{+} \Sigma^{-1}\left(\mu-\overline{x^{(\lambda)}}\right)\right\}\right\}|\Sigma|^{-\left(\frac{n-1}{2}\right)} \exp \left\{-\frac{1}{2} \operatorname{tr}[\Sigma\right. \\
& \exp \left\{\sum_{k=1}^{p} \sum_{i=1}^{n} \lambda_{k} \ln \left(x_{k i}\right)\right\}
\end{aligned}
$$

onde

$$
V=\sum_{i=1}^{n}\left(x_{i}^{(\lambda)}-\overline{x^{(\lambda)}}\right)\left(x_{i}^{(\lambda)}-\overline{x^{(\lambda)}}\right)^{\prime}
$$

As distribuições condicionais para o algoritmo Gibbs Sampling, são dadas por,

$$
\pi(\mu \mid \Sigma, \lambda, D) \propto \exp \left\{-\frac{n}{2}\left[\left(\mu-\overline{x^{(\lambda)}}\right)^{\prime} \Sigma^{-1}\left(\mu-\overline{x^{(\lambda)}}\right)\right]\right\}
$$

Portanto,

$$
\left(\mu \mid \Sigma_{2} \lambda_{2} D\right) \sim N_{p}\left(\bar{x}_{2(\lambda)} \frac{5}{n} \Sigma^{-1}\right)
$$

Para $\boldsymbol{\Sigma}$ temos,

$$
\begin{aligned}
\pi\left(\mathbf{\Sigma} \mid \mu_{2}, \lambda, D\right) & \propto|\mathbf{\Sigma}|^{-\frac{1}{2}(n+p+1)} \exp \left\{-\frac{1}{2} \sum_{i=1}^{n}\left[\left(x_{i}^{(\lambda)}-\mu\right)^{\prime} \Sigma^{-1}\left(x_{i}^{(\lambda)}-\mu\right)\right]\right\} \\
& \propto|\mathbf{\Sigma}|^{-\frac{1}{2}(n+p+1)} \exp \left\{-\frac{1}{2} \operatorname{tr}\left[\mathbf{\Sigma}^{-1} \mathbf{p}\right]\right\}
\end{aligned}
$$

onde

$$
\mathrm{P}=\sum_{i=1}^{n}\left(\mathrm{x}_{i-1}^{(\lambda)}-\mu\right)\left(\mathrm{x}_{i}^{(\lambda)}-\mu\right)^{\prime}
$$

Portanto,

$$
(\Sigma \mid \mu, \lambda, D) \sim I_{\boldsymbol{n}} u-\not{W} i s \bar{h} a r t_{n}\left(\mathrm{P}^{-1}\right)
$$

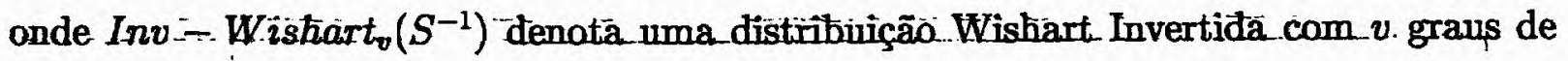
liberdade comn densidade,

$$
f(W) \propto \mid W L^{-\frac{1}{2}(v+q+1)} \exp \left\{-\frac{1}{2} \operatorname{tr}\left[S W^{-1}\right]\right\}
$$

$S$ é uma matriz escalar quadrādã $q \times q$, simétrica e définidā positiva e $W$ é definida positiva.

Finalmente, para $\lambda$ vamos ter,

$$
(\lambda \mid \Sigma, \mu, D) \propto \prod_{k=1}^{p} N\left(a_{k}, b_{k}\right) \exp \left\{-\frac{1}{2} \operatorname{tr}\left[\Sigma^{-1} \mathrm{P}\right]+\sum_{k=1}^{p} \sum_{i=1}^{n} \lambda_{k} \ln \left(x_{k i}\right)\right\}
$$




\section{5- Procedimentos de Simulaçãoe Diagnóstico de Con- vergência.}

De forma geral; para os algoritmos MCMC, podemos gerar uma ou várias cadeias independentes para cada parâmetro de interesse. Quando geramos várias cadeias, este procedimento evita que uma parte significativa dos espaço de parâmetros não seja explorado, sendo que o descarte inicial para cada cadeia protege contra estimativas viciadas devido a infflüêncià das condições ininiciäis( ver por exemplö, Gelman et al,1995). Na entanto pode-se também gerar uma única longa cadeia e descartar parte significativa da mesma. Verificado a convergência em ambos os casos, um ponto fundamental em simulação MCMC é a maneira como selecionamos as amostras obtidas. Temos, para cada cadeia gerada, que descartar uma quantidade ìniciat (burn-in) e tomar as observạ̧ōes restantes em saltos, por exemplo tomar de 10 em 10 observações, formando a amostra final selecionada. Este procedimento de obtenção de amostras é muito útil, principalmente para o algoritmo Metropolis-Hastinggs, poìs diminui a correlação entre os valöres repetidos devido rejeição durante o teste de aceitação.

Nós exemplos ilustrados, âplicamos os dois procedimentos, para a estimação a partir da verossimilhança perfilada usamos várias cadeias e para a verossimilhança completa usamos uma única longa cadeia.

Para o diagnóstico de convergência,no caso de várias cadeias, utilizamos o método de Gelman \& Rubin (ver Gelman \& Rubin; 1992) e o critério de Gewekēe (vèr Gewekej,j; 1992) para o caso_de uma única cađẹia.

\subsection{Alguns Exemplos- Numéricòs}

Nesta seção vamos nos voltar para os exemplòs do capítulo 2, efêtuando as transformaçōes dos dados via Bā̃ \& Cox, utilizanda o procedimento cléssico (TBox) e os procedimentōs Bayesianos (TBoxle TBō̄2).

Exemplo 3.1. Agora, vamos utilizar os dados do exemplo 2.2, aplicar os métodos de transformação Box-Cox clássico e o enfoque Bayesiano, realizar os procedimentos de classificação e verificar o ganho ou não na taxa de classificação para cada caso. 


\subsubsection{Estimação dos parâmetros de transformaçãa via Box-Cox Clássico (TBax).}

Para estimar os parâmetros de transformação $\lambda_{1}$ e $\lambda_{2}$, fizemos alguns testes através de inspeções gráficas e definimos o intervaló comum $[-5 ; 5]$. Este intervato fói particionàado de 0.01 em 0.01, totalizando 1000 pontos. Cada ponto foi testado na função perfilada e comparados, sendo selecionados os pontos que maximizarão a função. Foi usado o softwiare "MatLab" para a execução do algoritmo. Os resultados são mostrados na Tabela 3.1.

Tabela.3.1:Estimativas(TBQx)

\begin{tabular}{|cc|}
\hline$\lambda_{1}$ & $\lambda_{2}$ \\
\hline 0.45 & 0.12 \\
\hline
\end{tabular}

\subsubsection{Estimação dos parâmetros de transformação via Box \& Cox Bayesiano (TBox1).}

Para este procèdimento, assumimos as distribuiçõès a priori, descritas anteriormẹnte, sendo as escolha para os valores dos parâmetros e as condiçôes iniciais, feitas de fórma arbitrária, tomando como base várias simulações feitas ao longo do experimento:

Neste exemplo, geramos 5 cadeias de 2000 observações para cada parâmetro, des̈cartamos as 500 amostras iniciais de cada cadeia "burn-in"e tomamos o restantè de 10 em 10, totalizando uma amostra final de 150 observações para cada parâmetro. Nas Tabelas 3.2,3.3 e 3.4 temos os valores escolhidos para os parâmetros a priori, as condições iniciais e o resumos dos resultados.

Tabela 3.2:Parâmetros a pniori.

\begin{tabular}{|c|c|c|}
\hline parâmetros & $a_{k}$ & $b_{k}$ \\
\hline$\lambda_{1}$ & 0.45 & 0.60 \\
$\lambda_{\overline{2}}$ & -0.12 & 0.25 \\
\hline
\end{tabular}

Tabela 3.3:Condições Iniciais

\begin{tabular}{|l|lclll|}
\hline$\lambda_{1}$ & 0.3 & 0.3625 & 0.425 & 0.4875 & 0.55 \\
$\lambda_{2}$ & -0.05 & -0.0125 & 0.0250 & 0.0625 & 0.1 \\
\hline
\end{tabular}


Tabela 3.4:Resumo dos Resultados

\begin{tabular}{|cccccccc|}
\hline & Mëdia & Moda & Dësvio & (inf.)IC 95\% & (sup.)IC 95\% & GR & Aceitação \\
\hline$\lambda_{1}$ & 0.486 & 0.52 & 0.2828 & -0.0667 & 1.0033 & 1.0012 & $55.30 \%$ \\
$\lambda_{2}$ & 0.139 & 0.135 & 0.153 & -0.1667 & 0.436 & 0.9984 & $67.15 \%$ \\
\hline
\end{tabular}

Nos procedimentos Bayesianos, damos particular importância as estatísticas referentes ao critério dè convergência, aqui utilizamos o critếrio dē Gelman \& Rubin "GR"; que neste caso se mostrou plenamente satisfatório, próximo de 1, o que garante a convergência e também quando trabalhamos com o algoritmo Metropolis-Hastings, nos preocupamos com a taxa de aceitação, que neste caso também se mostrou aceitável. Abaixo nas Figuras 3.1 e 3.2; temos os gráficas de convergência e as densidades marginais a posteriori.

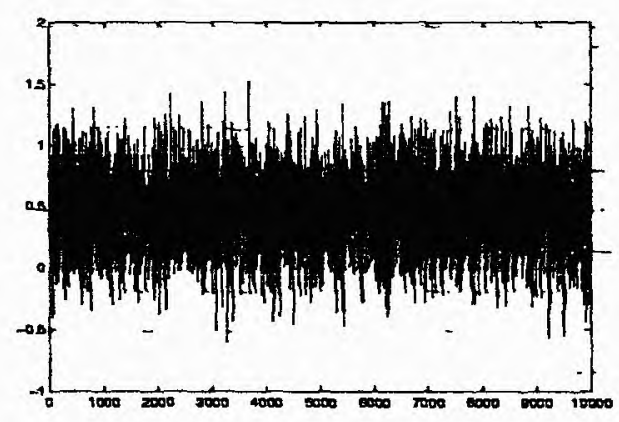

$\lambda_{1}$

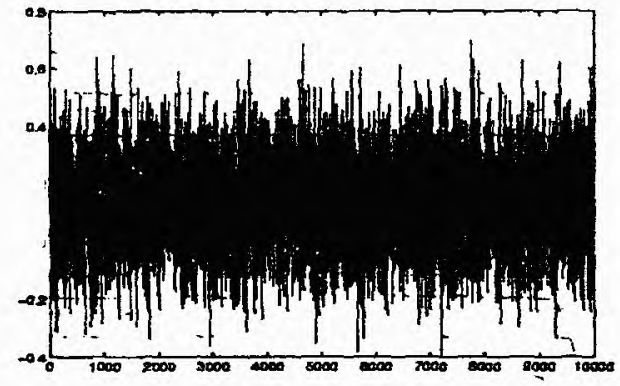

$\lambda_{2}$

Figura 3.1: Convergência para os parâmetros.

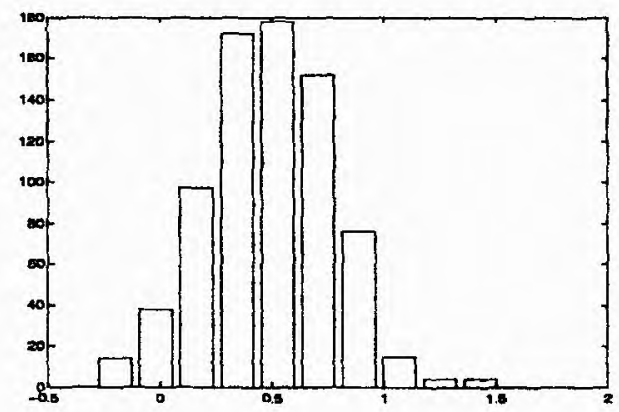

$\lambda_{1}$

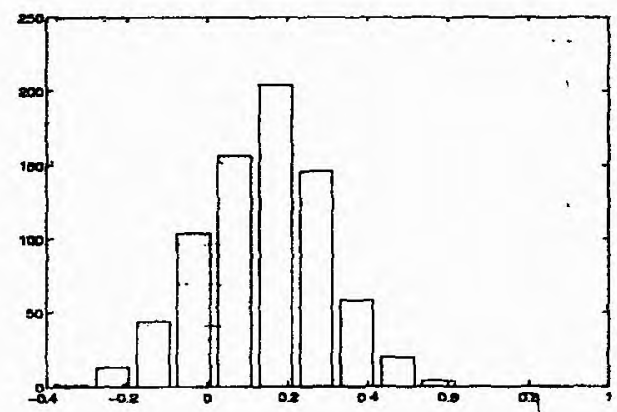

$\lambda_{2}$

Figura 3.2: Densidades Marginais a Posteriori 


\subsubsection{Estimação dos parâmetros de transformação via Box \& Cox Bayesiano (TBox2).}

Nesta seção tentamos propor uma outrāa altërnativa para o problema de transformação. No entanto não fomos muito felizes, com relação ao procedimento usado e aos resultădos obtidos.

\section{Dificuldades Surgidas}

Percebemos alguns problemas neste procedimento para estimarmos os parâmetros de transformação. A convergência requerida pelo método MCMC, só foi conseguida quando atribuímos uma distribuição a priori para os parâmetros muito informativa, ou \$̦eja, com variância muito baixa. Outro fato que nos preocupa e que percebemos an longó da execução do algoritmo é que as estimativas de $\boldsymbol{\Sigma}$ e $^{-} \boldsymbol{\mu}$ variam muito de acordo corp os parâmetros atribuídos para as distribuições a priori dos $\lambda$ 's, sempre convergindo. Enfim, é um procedimento que ainda nos gera dúvidas, portanto não aconselhamos seu uso da forma apresentada.

\section{Procedimento de Estimação}

Para estimação dos parâmetros utilizamos o aigoritmo Gibbs Sampling com MetropplisHastings, onde geramos uma única grande cadeia com 10000 valores, descartamos os 4000 valores iniciais e tomamos de 20 èm 20 totalizzando üma amostra final de 300 valores para cada parâmetro. Como temos uma cadeia, utilizamos o critério de convergência dè Gewekè (ver Geweke,J;1992).Nas Tabelas 3.5,3.6 e 3.7 temos as condições iniciais, os valores escolhidos para os parâmetrös a priori e o resumos dos resultados.

Tabela 3.5:Condições Iniciais

\begin{tabular}{|c|c|c|c|c|c|c|}
\hline$\lambda_{1}$ & $\lambda_{2}^{-}$ & $\mu_{1}$ & $\mu_{2}$ & $\sigma_{1}^{2}$ & $\sigma_{2}^{2}$ & $\sigma_{12}$ \\
\hline 0.42 & 0.11 & 10 & 2.2 & 6 & -0.5 & 0.6 \\
\hline
\end{tabular}

Tabela 3.6:Parâmetros a priori.

\begin{tabular}{|c|c|c|}
\hline parâmetros & $a_{k}$ & $b_{k}$ \\
\hline$\lambda_{1}$ & 0.46 & 0.005 \\
$\lambda_{2}$ & 0.13 & 0.0065 \\
\hline
\end{tabular}


Tabela 3.7:Resumo dos Resultados

\begin{tabular}{|ccccccc|}
\hline & Média & Desviò & (inf.)IC 95\% & (sup.)IC 95\% & GW & Aceitação \\
\hline$\lambda_{1}$ & 0.4673 & 0.0053 & 0.4569 & 0.4772 & 0.1992 & $33.79 \%$ \\
$\lambda_{2}$ & 0.1360 & 0.0064 & 0.1240 & 0.1483 & 0.0266 & $47.24 \%$ \\
$\mu_{1}$ & 10.44 & 0.294 & 9.9065 & 11.07 & 0.0263 & - \\
$\mu_{2}$ & 2.66 & 0.09 & 2.50 & 2.85 & 0.033 & - \\
$\sigma_{1}^{2}$ & 6.025 & 1.1437 & 4.29 & 8.78 & 0.085 & - \\
$\sigma_{2}^{2}$ & 0.72 & 0.13 & 0.50 & 1.026 & 0.01 & - \\
$\sigma_{12}$ & -0.85 & 0.2961 & -1.50 & -0.32 & 0.019 & - \\
\hline
\end{tabular}

Neste caso, conseguimos bons resultados para o "GW"e para a taxa de rejeição. Nas Figuras 3.3 e 3.4, temos os gráficos de convergência e as densidades marginais a posteriori.

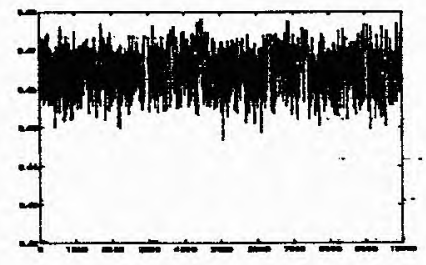

$\lambda_{1}$

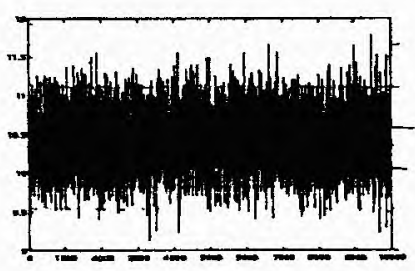

$\mu_{1}$

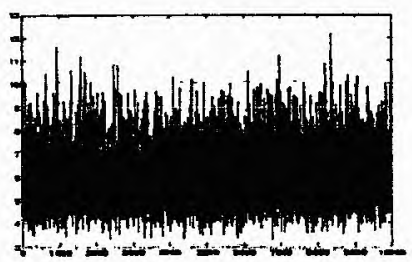

$\sigma_{1}^{2}$
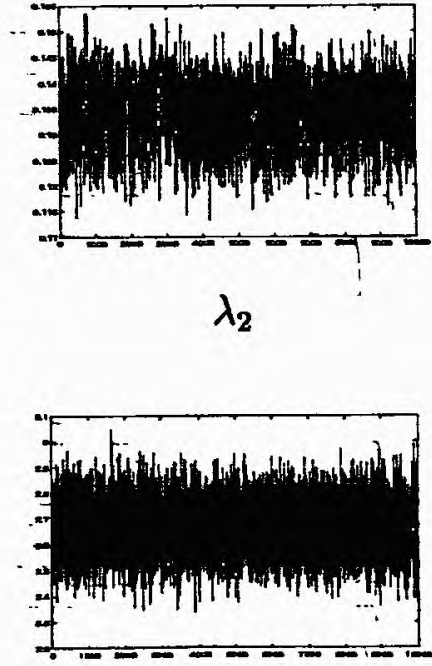

$\mu_{2}$

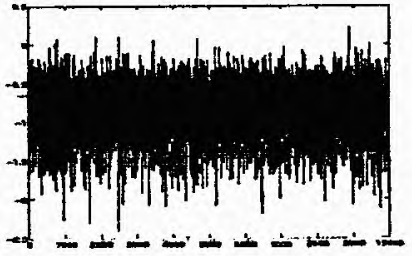

$\sigma_{12}$

Figura 3.3: Cōuvergência para os parâmetros. 


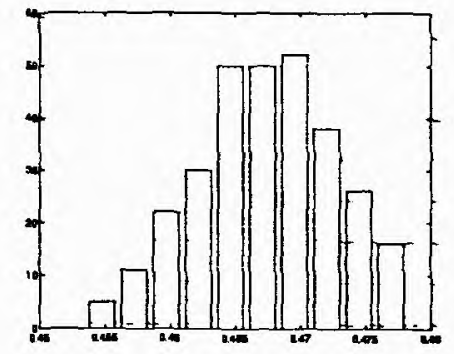

$\lambda_{1}$

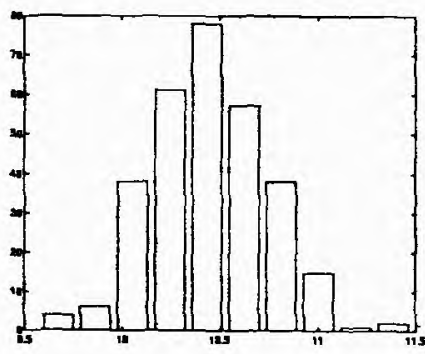

$\mu_{1}$

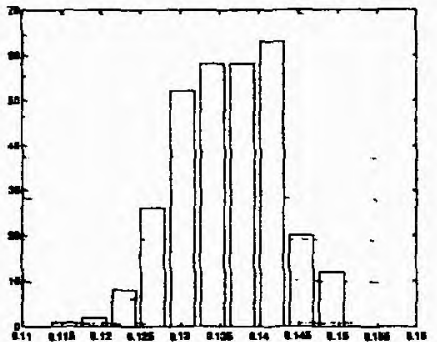

$\lambda_{2}$

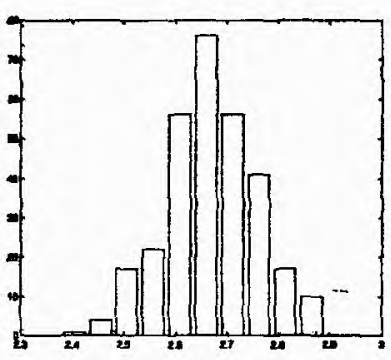

$\mu_{2}$

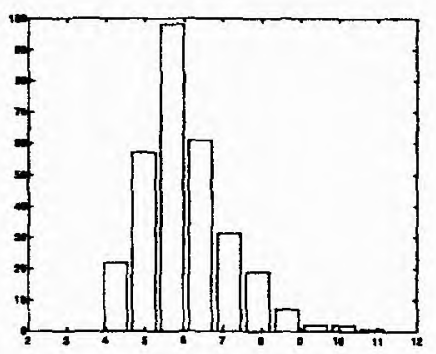

$\sigma_{1}^{2}$

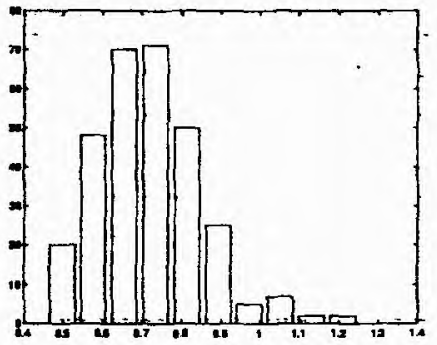

$\sigma_{2}^{2}$

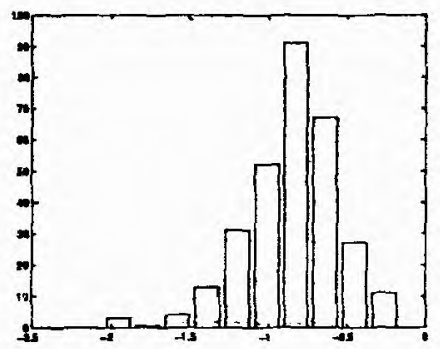

$\vec{\sigma}_{12}$

Figura 3.4: Densidades Marginais a Posteriori.

\subsubsection{Comparação das transformações e dos procedimentos de} Classificação.

Estimados os parâmetros de transformação por todos os procedimentos propostos, verificamos visualmente através do Qui-Quadrado Plat (ver Johnson \& Wichern;1982) a normalidade multivariada para os dados sem transformação e com transformação. 


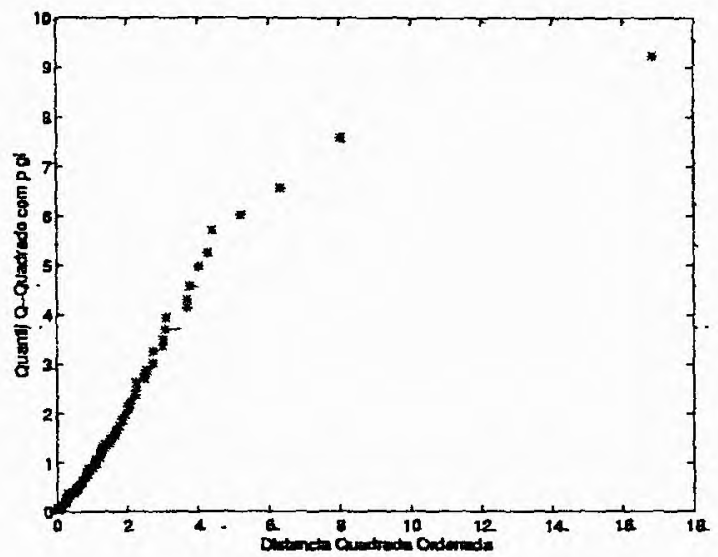

DST

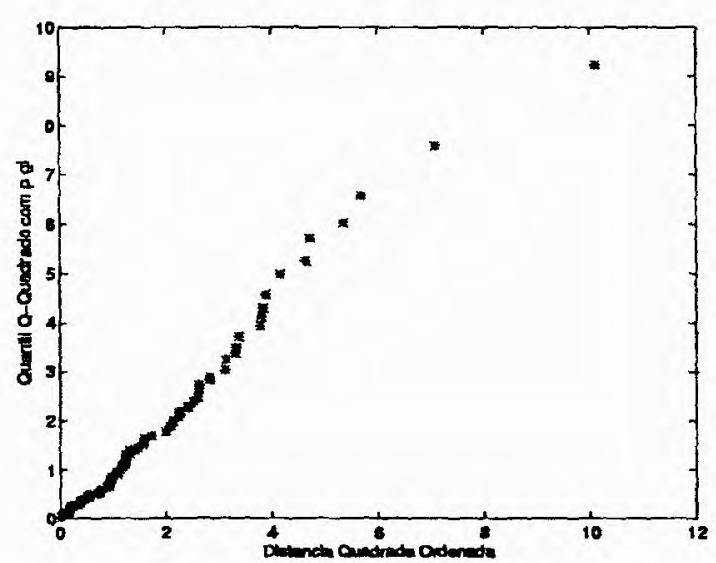

TBox1

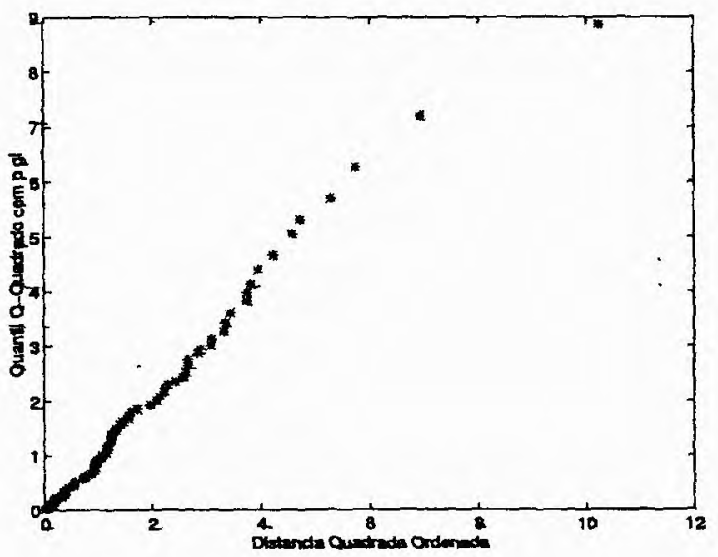

TBox

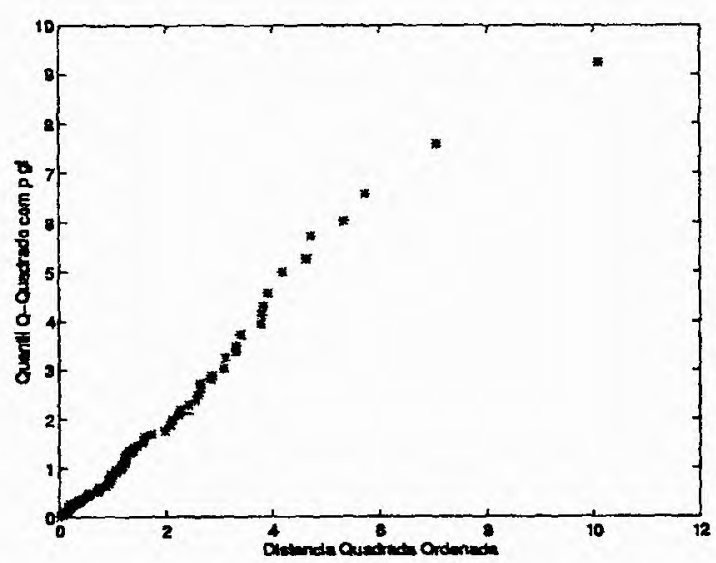

TBox2

Figura 3.5: Teste de Normalidade Multivariada $p=2$.

O teste acima, nos leva a acreditar que quando aplicamos os procedimentos de transformação a normalidade melhora, não tendo muita distinção entre os procedimentos propostos. Abaixo na Tabela 3.8, verificamos o desempenho dos critérios de classificação(F.D.L. e F.D.Q.) quando utilizamos os diferentes procedimentos de transformacão

Tabela 3.8: Taxa de Erro Aparente (APER)

\begin{tabular}{c|c|c|c|c|}
\hline & DST & Tbox & Tbox1 & Tbox2 \\
\hline FDL & 0.2254 & 0.2394 & 0.2394 & 0.2394 \\
FDQ & 0.2535 & 0.2113 & 0.2113 & 0.2113 \\
\hline
\end{tabular}


Verificamos que em todas as transformaçōes, os efeitos nas taxas de classificação foram os mesmos para os procedimentos ùsadōs. No caso da F.D.L., dado as condições iniciais $\left(\frac{c(1 / 2)}{c(2 / 1)}\right)\left(\frac{p_{2}}{p_{1}}\right)=1$, vamos acabar caindo na Função Discriminante Linear de Fisher. Neste caso, o fator normalidade não necessariamente melhora os resultados da classificação. Percebemos que as taxas de erro aumentaram, no entanto, com a normalidade temos propriedades ótimas que poderão garantir melhor estes resultados.

Já para F.D.Q., o fator normalidade e a condição de que as matrizes de covariâncias sejam diferentes são essenciais para uma boa classificação. Percebemos que neste caso a taxa de classificação errada diminuiu. Em relação aas métodos de transformação não houve nenhum destaque.

\subsubsection{Resultados para dados transformados de ordem $p>2$.}

Nesta seção vamos apresentar os resultados da classificação com dados transformados para os dados do exemplo 3.2 .

Da mesma forma, estimamos $\lambda_{1}, \lambda_{2}, \ldots, \lambda_{5}$, que maximizam (3.10, utilizando as transformações Box \& Cox Clássico(Tbox), Box \& Cox Bayesiano a partir da função de Verossimilhança Perfilada (TBox1) e Box \& Cox Bayesiano a partir da função de Verossimilhança Completa(TBox2).

O resumos das taxas de classificação estão dảdos nà Tabela 3.9 abaixo:

\begin{tabular}{c|c|c|c|c|}
\multicolumn{5}{c}{ Tabelà 3.9:Taxa de Erro Aparente (APER) } \\
\hline & DST & Tbox & Tbox1 & Tbox2 \\
\hline FDL & 0.071 & 0.107 & 0.089 & 0.089 \\
FDQ & 0.036 & 0.018 & 0.018 & 0.018 \\
\hline
\end{tabular}

Novamente temos um melhor desempenho para a classificação dos dados transformados quando usamos a F.D.Q., que acreditamos se dar pelo mesmo motivo anterior. No geral conseguimos melhorar a taxa de classificação a partir da transformação. No entanto, não conseguimos detectar um melhor procedimento.

Como foi comentado anteriormente, a maximização marginal para a transformação (TBox) em dados multivariados pode ser prejudicial em alguns casos. Aqui, com os exemplos ilustrados, não conseguimos verificar tal fato. No entanto mostramos a igual 
eficiência para o procedimento Bayesiano (TBox1) e constatamos a facilidade em sua implementação e execução. Na nossa opinião este é um procedimento mais completo, pois efetua a maximização conjunta para dados multivariados. Assim, usando tal procedimento, não precisamos nos preocupar com o efeito da correlação ou não entre as variáveis de uma observação multivariada, portanto sugerimos a sua utilização.

No próximo capítulo trataremos o problema de classificação, considerando o vetor aleatório $\mathrm{x}$ de medidas como tendo uma mistura de distribuições normais multivariadas. 


\section{Capítulo 4}

\section{Classificação Considerando a}

\section{distribuição do vetor de medidas $X$, como uma Misturas de Distribuições}

Neste capítulo tratamos o problema de classificação, considerando a distribuição do vetor aleatório $\mathrm{X}$ de medidas, como uma mistura de duas distribuições normais multivariadas, tendo como objetivo, para algumas situações, adequar melhor o modelo aos dados e conseqüentemente melhorar a taxa de classificação.

\subsection{Introdução aos Modelos de Misturas}

Mödelos estatísticos baseados em distribuições mistas formam uma classe de modelos, que se ajustam naturalmente quando são aplicados à observações amostrais obtidas de diferentès situações, isto é quando a população é heterogênea. Essas siturações são encontradas com grande freqüência no nosso cotidiano. Por exemplo, a distribuição das alturas em uma população adulta refléte a mistura de hömens e mulkeres. Os modelòs Bayesianos, baseados em distribuições mistas, estão sendo muito utilizados recentemente, apresentando certo sucesso, especialmente devido a förmulação dè novos algoritmos computacionais e pela forma natural de interpretar as diferentes situações em que o experimento é realizado.

No problema de classificação, a utilização da modelàgem Bayesiana para os modelos de misturas se mostra bastante interessante, pois em várias situações tal procedimento poderá modelar melhor os dados de cada população específica, conseqüentemente, podemos ter 
melhores resultados na classificação de novas observações.

Pàra o problema de classificação, normalmente se associa ao vetor aleatớrio $X$ uma mistura de distribuições normais. multivariadas (ver por exemplo,Lavine \& West,1992). Assima, para cada população temos,

$$
f(\mathrm{x} \mid \boldsymbol{\theta})=\sum_{j=1}^{K-} p_{j} f\left(\mathrm{x} \mid \boldsymbol{\theta}_{j}\right)
$$

onde $\boldsymbol{\theta}_{j}$ é um vetor de parâmetros associado comュ $j$-ésimo componente da distribuiçãa e $\sum_{j=1}^{2} P_{j}=1$.

A inferência Bayesiana para mistura de distribuições é introduzida por vários autores (ver por exemplö, Robert,1996; ou Titterington, Simith e Màkov,1985).

Como um caso especial, consideramos uma alternativa Bayesiana para a classificação, assumindo uma mistura de distribuições normais multivariadas para cada populaçã̉o e usamos os métodōs de simutação MCMC(Monte Carto em Cádeias dê Markov) para o desenvolvimento das regras de clàssificação.

\subsection{Añálisèe Bayesiāna Assumindo uma Mistura de $K=2$ Distribuiçōes Normais Mültivariadàs}

Aquui, para cada população associamos uma mistura de duas densidades normais multivariadas para o vetor $\mathrm{X}$, representando a contribuição de duas sub-populações na amostra. Assim, a dēnsidade será escrita na forma,

$$
f(\ddot{x} \mid \theta)=\sum_{j=1}^{2} p_{j} f_{j}\left(\bar{x} \mid \theta_{j}\right)
$$

onde $\mathrm{x}=\left(x_{1}, \ldots, x_{i}\right) ; \sum_{j=1}^{2} p_{j}=1$, sendo que $f_{j}\left(x \mid \theta_{j}\right)$ deneta uma distribuição normal moultivariadà $N_{q}\left(\mu_{j}, \Sigma_{j}\right)$, señda $\theta=\left\{\boldsymbol{\theta}_{1}, \theta_{2}\right\}$, ondè $\theta_{1}=\left\{\mu_{1}, \Sigma_{1}\right\}, \theta_{2}=\left\{\mu_{2}, \Sigma_{2}\right\}$ e $\mathrm{p}=\left\{p_{1}, p_{2}\right\}$

A funçãa de verossimilhança para $\theta$ e $p=\left(\ddot{p}_{1}, p_{2}\right)$ baseado em uma amostra aleaț́ria $\mathrm{X}_{1}, \mathrm{X}_{2}, \ldots, \mathrm{X}_{n}$ é dada por

$$
L(\theta, \mathrm{p})=\prod_{i=1}^{n}\left\{\sum_{j=1}^{2} p_{j} f_{j}\left(\mathrm{x}_{i} \mid \boldsymbol{\theta}_{j}\right)\right\}
$$


Atribuindö diștribuições a priori $\pi(\theta)$ e $\pi(p)$ para os vetores de parâmetros $\theta$ ę $p$, respectivamente e considerando-as independeñtes, temos a fünção de đistribuição a posteriori dada por,

$$
\pi(\theta, \mathrm{p} \mid \mathrm{X}) \propto \pi(\boldsymbol{\theta}) \pi(\mathrm{p}) L(\theta, \mathrm{p})
$$

Percebemos que tal forma, independente da distribuição a priori utilizada, geralmente apresenta uma grande dificuldade na obtenção das densidades a posteriori marginais dos parâmetros do modelo, devido a complexidade dafunção de verossimilhança. Uma solụção para o. problema é a utilização dâ variável latènte (vier Tañner e Wong, 1987); quẹ irá transformar o modèlo de misturas em um modètó de componentes indèpendèptes.

\subsubsection{Inçorporação da Variável Latente}

As̀ variáveis lätentes são incorporadā̄ no modelō com o objetivo de simplificar a distribuição a posteriori facilitando o trabalho computacional. Assim, definimos a seguinte variável.nãa öbservada $Z_{i}=\left(Z_{i 1}, Z_{i 2}\right)$; sepdo,

$$
Z_{i j}=\left\{\begin{array}{l}
\ddot{1} \text { se a } i \text {-ésima obs. foi gerada da pop. } j, \ldots \\
0 . \\
\text { caso contrário, }
\end{array}\right.
$$

onde $\cdot \sum_{j=1}^{2 \cdots} Z_{i j}=1$, para $i=1, \ldots, n$.

Neste caso específico para $K=2$ componentes da distribuição temos,

$$
\left(Z_{i j} \mid \mathrm{X}, \boldsymbol{\theta}, \dddot{\mathrm{p}}\right) \sim \operatorname{Bin}\left(1 ; v_{i j}\right)(\text { uma diștribuiçãa binomial }),
$$

onde $v_{i j}$ é dèfinido como,

$$
v_{i j}=\frac{p_{j} f_{j}\left(\mathrm{x}_{i} \mid \boldsymbol{\theta}_{j}\right)-\bar{c}}{\sum_{j=1}^{2} p_{j} f_{j}\left(\mathbf{x}_{i} \mid \theta_{j}\right)}
$$

Assim,

$$
f\left(\mathrm{z}_{i} \mid \mathrm{X}, \boldsymbol{\theta}, \mathrm{p}\right) \cdot=v_{i 1}^{z_{i 1}} v_{i 2}^{z_{i 2}}=v_{i 1}^{z_{i 1}}\left(1-v_{i 1}\right)^{1=z_{i 1}}
$$

Pàra uma amostra $Z_{1}, \ldots, Z_{n}$, temos 


$$
\begin{aligned}
& L(\mathrm{z} \mid \mathrm{x}, \boldsymbol{\theta}, \mathrm{p})=\prod_{i=1}^{n-}\left\{\left(\frac{p_{1} f_{1}\left(\bar{x}_{i} \mid \theta_{1}\right)}{\sum_{j=1}^{2} p_{j} f_{j}\left(\mathrm{x}_{i} \mid \theta_{i}^{-}\right)}\right)^{z_{i 1}}\left(\frac{p_{2} f_{2}\left(\mathrm{x}_{i} \mid \boldsymbol{\theta}_{2}\right)^{-}}{\sum_{j=1}^{2} p_{i} f_{j}\left(\mathrm{x}_{i} \mid \theta_{j}\right)}\right)^{x_{i 2}}\right\} \\
& =\frac{\prod_{i=1}^{n} \Pi_{j=1}^{2}\left[p_{j} f_{j}\left(x_{i} \mid \theta_{j}\right)\right\}^{z_{i j}}}{\prod_{i=1}^{n}\left\{\sum_{j=1}^{2} p_{j} f_{j}\left(x_{i} \mid \theta_{j}\right)\right\}},
\end{aligned}
$$

onde $\mathrm{z}=\left\{\bar{z}_{1}, \ldots, \ldots, \overline{z_{n}}\right\}$.

Portanto, a função de verossimilhança incorporada pelos dados ampliados será dada por:

$$
\begin{aligned}
L(\mathrm{x}, \mathrm{z} \mid \boldsymbol{\theta}, \overline{\mathrm{p}}) & =L(\overline{\mathrm{x}} \mid \boldsymbol{\theta}, \mathrm{p}) L(\mathrm{z} \mid \mathrm{x}, \boldsymbol{\theta} ; \mathrm{p}) \\
& =\prod_{i=1}^{n}\left\{\left[p_{1} f_{1}\left(\mathrm{x}_{i} \mid \theta_{1}\right)\right]^{z_{i 1}}\left[p_{2} f_{2}\left(\mathrm{x}_{i} \mid \boldsymbol{\theta}_{2}\right)\right]^{z_{i 2}}\right\} .
\end{aligned}
$$

Definidà a verossimilhança com a incorporação da variável Latente, mostramos a seguir a incorporação das informações a priori e a determinação da distribuição posteriori.

\section{3 - Distribuições a Priori para o Modelo de Mistura}

Como sempre, uma das grandes dificuldàdes dāinferência Bayesiana é incorporar a informação a priori no modelo quando não temos a opinião de um especialista. A escolba da dènsidāde a priời é feita dè fơrma a se tēr uma distribüição a posteriori tratável. Nōs modèlos de mistura onde $f_{j}\left(\mathbf{x} \mid \theta_{j}\right)$ pertëncem a familia exponencial; àlém de usarmos a distribuição a priori não informatîva (Priori de Jeffrey), tāmbèm podẹmos usar a distribuiçãa a priori conjugada, ver por exempla Räbert,C.P(1996). Nesste trabalho incorporamos prioris não införmativas e prioris conjugadas para o modēto e verificamos $o$ desempenho destes para o problema de classificação.

\subsubsection{Desenvolvimento do Modelo com Distribuição a Priori Não Informativa \\ O modelo Bayesiano de misturas normais multivariadas; utilizando prioris não infor- mativas, está bem ilustrado em (Lavine,1994). Neste vamos ter prioris não informativas para os parâmetros dados abaixo,}




$$
\begin{aligned}
& \pi(\theta)=\left|\mathbf{\Sigma}_{1}\right|^{-\frac{1}{2}(q+1)}\left|\mathbf{\Sigma}_{2}\right|^{-\frac{1}{2}(q+1)} . \\
& \pi(p)=\text { Beta }(a, b), \text { onde } a, b \text { conhecidos. }
\end{aligned}
$$

Súpondo indèpendência a prióri, teremos a densidade posteriori dađà por,

$$
\begin{aligned}
& \pi(\boldsymbol{\theta}, \mathrm{p} \mid \mathrm{x}, \mathrm{z}) \propto \pi(\boldsymbol{\theta}) \pi(\mathrm{p}) p_{1-}^{\sum_{i=1}^{\mathrm{n}} z_{i 1}} p_{2}^{\sum_{i=1}^{\mathrm{n}} z_{i 2}} \prod_{i=1}^{n-}\left\{\left[f_{1}\left(\mathrm{x}_{i} \mid \boldsymbol{\theta}_{1}\right)\right]^{z_{i 1}}\left[f_{2}\left(\mathrm{x}_{i} \mid \boldsymbol{\theta}_{2}\right)\right]_{\cdots 1}^{z_{i 2}}\right\} \\
& \propto\left|\mathbf{\Sigma}_{1}\right|^{-\frac{1}{2}(q+1)} \prod_{i=1}^{n}\left\{\left[f_{1}\left(\mathbf{x}_{i} \mid \boldsymbol{\theta}_{1}\right)\right]^{z_{i 1}}\right\}\left|\mathbf{\Sigma}_{2}\right|^{-\frac{5}{2}(q+1)} \prod_{i=1}^{n}\left\{\left[f_{2}\left(\mathbf{x}_{i} \mid \boldsymbol{\theta}_{2}\right)\right]^{z_{i 2}}\right\} \\
& p_{1}^{(r \pm a)-1}\left(1-p_{1}\right)^{(n-r+b)-1}
\end{aligned}
$$

onde $p_{2}=1-p_{1}, r=\sum_{i=1}^{n} z_{i 1}$ e. $n-r=\sum_{i=1}^{n \cdot-} z_{i 2}$.

\subsubsection{Distribuições Condicionais a Posteriori Necessárias para o Algoritmo Gibbs. Sampling}

Dà expressãō (4.9), vamos derivar as distribiuiçôes condicionais necessárias para gerarmos amostras via simuläção MCMC para a análise Bayesiana. Assim,

$$
\pi(p \mid \theta, x, z) \propto p_{1}^{(r+a)-1}\left(1-p_{1}\right)^{(n-r+b)-1}
$$

Portanto,

$$
(\mathrm{p} \mid \boldsymbol{\theta}, \overline{\mathrm{x}}, \mathrm{z}) \sim \operatorname{Beta}(r+a ; n-r+\bar{b})
$$

Para $\mu_{1}$ temos,

$$
\begin{aligned}
\pi\left(\mu_{1} \mid \Sigma_{1}, p_{1}, \theta_{2}, \mathrm{x}, \mathrm{z}\right) & \propto \prod_{i=1}^{n}\left[\left|\Sigma_{1}\right|^{-\frac{1}{2}} \exp \left\{-\frac{1}{2}\left(\mathrm{x}_{i}-\mu_{1}\right)^{\prime} \Sigma_{1}^{-1}\left(\mathrm{x}_{i}-\mu_{1}\right)\right\}\right]^{z_{i 1}} \\
& \propto\left|\Sigma_{1}\right|^{-\frac{r^{-}}{2}} \exp \left\{-\frac{1}{2} \sum_{i=1}^{n} z_{i 1}\left(\mathrm{x}_{i}-\mu_{1}\right)^{\prime} \Sigma_{1-}^{-1}\left(\mathrm{x}_{i}-\mu_{1}\right)\right\} \\
& \propto \exp \left\{-\frac{1}{2} \sum_{i=1}^{n} z_{i 1}\left[\mathrm{x}_{i}^{\prime} \Sigma_{1}^{-1} \mathrm{x}_{i}-\mu_{1}^{\prime} \Sigma_{1}^{-1} \mathrm{x}_{i}-\mathrm{x}_{i}^{\prime} \Sigma_{1}^{-1} \mu_{1}+\mu_{1}^{\prime} \Sigma_{1}^{-1} \mu_{1}\right]\right\} \\
& \propto \exp \left\{-\frac{r}{2}\left[\mu_{1}^{\prime} \Sigma_{1}^{-1} \mu_{1}-2 \mu_{1}^{\prime} \Sigma_{1}^{-1} \frac{1}{r} \sum_{i=1}^{n} z_{i 1} \mathbf{x}_{i}+\frac{1}{r} \sum_{i=1}^{n-} z_{i 1}\left(x_{i}^{\prime} \Sigma_{1}^{-1} x_{i}\right)\right]\right\}
\end{aligned}
$$


Fazendo $\cdot \frac{1}{r} \sum_{i=1}^{n} z_{i 1} x_{i}=\bar{x}_{1}$, temos,

$$
\begin{aligned}
& \pi\left(\mu_{1} \mid \boldsymbol{\Sigma}_{1}, p_{1}, \boldsymbol{\theta}_{2}, \mathrm{x}, \mathrm{z}\right) \propto \exp \left\{-\frac{F}{2}\left[\boldsymbol{\mu}_{1}^{\prime} \boldsymbol{\Sigma}_{1}^{-1} \boldsymbol{\mu}_{1}-2 \boldsymbol{\mu}_{1}^{\prime} \boldsymbol{\Sigma}_{1}^{-\mathrm{t}} \bar{x}_{1}+\bar{x}_{1}^{\prime} \boldsymbol{\Sigma}_{1}^{-\mathrm{t}} \bar{x}_{1}+\frac{1}{r} \sum_{i=1}^{n} \cdot \bar{z}_{i 1}\left(\mathbf{x}_{i}^{\prime} \boldsymbol{\Sigma}_{1}^{-1} \mathbf{x}_{i}\right)-\bar{x}_{1}^{\prime} \boldsymbol{\Sigma}_{1}^{-1} \bar{x}_{1}\right]\right\} \\
& \left.\propto \exp \left\{-\frac{r}{2}\left(\mu_{1}-\bar{x}_{1}\right)^{\prime} \Sigma_{1}^{-1}\left(\mu_{1}-\bar{x}_{1}\right)\right\} \exp \left\{\frac{I}{2} \sum_{i=1}^{n-} z_{i 1}\left(x_{i}^{\prime} \dot{\Sigma}_{1}^{-1} \bar{x}_{i}\right)-r \bar{x}_{1}^{\prime} \Sigma_{1}^{-1} \overline{\bar{x}}_{1}\right]\right\}
\end{aligned}
$$

Assim podemos escrever,

$$
\pi\left(\mu_{1} \mid \Sigma_{1}, p_{1}, \theta_{2}, x_{2} z\right) \propto \exp \left\{-\frac{r}{2}\left(\mu_{1}-\bar{x}_{1}\right)^{\prime} \Sigma_{1}^{-1}\left(\mu_{1}-\bar{x}_{1}\right)\right\}
$$

Portanto,

$$
\left(\boldsymbol{\mu}_{1} \mid \mathbf{\Sigma}_{1}, p_{1}, \boldsymbol{\theta}_{2}, \mathbf{x}, \mathbf{z}\right) \sim N_{\mathbf{Q}}\left(\underline{\tilde{x}_{1}} ; \frac{\mathfrak{b}}{r} \mathbf{\Sigma}_{1}\right)
$$

Para $\boldsymbol{\Sigma}_{1}$ temos que,

$$
\begin{aligned}
& \pi\left(\Sigma_{1} \mid \mu_{1}, p_{1} \theta_{2}, x, z\right) \propto \int\left|\Sigma_{1}\right|^{-\frac{1}{2}(q+1)}\left|\Sigma_{1}\right|^{-\frac{\tau}{2}} \exp \left\{-\frac{1}{2} \sum_{i=1}^{n} z_{i 1}\left(x_{i}-\mu_{1}\right)^{\prime} \Sigma_{1}^{-1}\left(x_{i}-\mu_{1}\right)\right\} d \mu_{1} \\
& \left.\propto \Sigma_{1}\right|^{-\frac{1}{2}(\dot{q}+1)}\left|\Sigma_{1}\right|^{-\frac{r-1}{2}} \exp \left\{-\frac{1}{2} \cdot \sum_{i=1}^{n} z_{i 1}\left(x_{i}^{\prime} \Sigma_{1}^{-1} x_{i}\right)-r\left(\bar{x}_{1}^{\prime} \Sigma_{1}^{-1} \bar{x}_{1}\right)\right\} \\
& \int\left|\boldsymbol{\Sigma}_{1}\right|^{-\frac{1}{2}} \exp \left\{=\frac{r}{2}\left(\mu_{1}-\bar{x}_{1}\right)^{\prime} \boldsymbol{\Sigma}_{1}^{-1}\left(\boldsymbol{\mu}_{1}-\bar{x}_{1}\right)\right\} d \mu_{1}
\end{aligned}
$$

Assim temos,

$$
\pi\left(\Sigma_{1} \mid \mu_{1}, p_{1} \theta_{2}, \mathbf{x}, \mathbf{z}\right) \propto\left[\left.\Sigma_{1}\right|^{-\frac{1}{2}([r-1]+q+1)} \exp \left\{-\frac{1}{2} \operatorname{tr}\left[\Sigma_{1}^{-1} V_{1}\right]\right\}\right.
$$

onde $V_{1}=\sum_{i=1}^{n} z_{i 1}\left(x_{i}-\bar{x}_{1}\right)\left(x_{i}-\bar{x}_{1}\right)^{\prime}$.

Portainto,

$$
\left(\Sigma_{1} \mid \mu_{1} ; p_{1} \theta_{2}^{-}, x, z\right) \sim I n v-W i s \hbar a ̈ r t_{r-1}\left(V_{1}^{-1}\right)
$$

Dä mesma forma, vamos obtèr as marginais para $\mu_{2}$ e $\mathbf{\Sigma}_{2}$. Assim,

$$
\pi\left(\mu_{2} \mid \Sigma_{2}, p_{1}, \theta_{1}, \mathrm{x}, \mathrm{z}\right) \propto \exp \left\{-\frac{r_{2}}{2}\left(\mu_{2}-\bar{x}_{2}\right)^{\prime} \mathbf{\Sigma}_{2-}^{-1}\left(\mu_{2}-\bar{x}_{2}\right)\right\},
$$

onde $r_{2}=n-r$ e $\bar{x}_{2}=\frac{1}{r_{2}} \sum_{i=1}^{n} z_{i 2} x_{i}$.

Portanto,

$$
\left(\boldsymbol{\mu}_{2} \mid \mathbf{\Sigma}_{2}, p_{1}, \boldsymbol{\theta}_{1}, \mathbf{x}, \mathbf{z}\right) \sim N_{q}\left(\bar{x}_{2} ; \frac{1}{r_{2}} \Sigma_{2}\right)
$$

Para $\boldsymbol{\Sigma}_{\mathbf{2}}$ temos que, 


$$
\pi\left(\boldsymbol{\Sigma}_{2} \mid \boldsymbol{\mu}_{2}, p_{1}, \boldsymbol{\theta}_{1}, \mathbf{x}_{2} \mathrm{z}\right) \propto\left|\boldsymbol{\Sigma}_{2}\right|^{-\frac{1}{2}\left(\left[\boldsymbol{r}_{2}-1 \mathrm{f}+q+1\right)\right.} \exp \left\{-\frac{I}{2} \operatorname{tr}\left[\boldsymbol{\Sigma}_{2}^{-1} \mathbf{V}_{2}\right]\right\},
$$

onde $\mathrm{V}_{2}=\sum_{i=1}^{n} z_{i 2}\left(\mathrm{x}_{i}-\bar{x}_{2}\right)\left(\mathrm{x}_{i}=\bar{x}_{2}\right)^{\prime}$.

Portanto,

$$
\left(\boldsymbol{\Sigma}_{2} \mid \boldsymbol{\mu}_{2}, p_{1}, \boldsymbol{\theta}_{1}^{-}, \mathrm{x}_{7} \mathrm{z}\right) \sim \operatorname{In} v-W \cdot \text { ishart }_{\mathrm{r}^{-1}-1}\left(\mathbf{V}_{2}^{-1}\right)
$$

onde Inv - Wishart ${ }_{v}\left(S_{-1}^{-1}\right)$ denota uma distribuiçãa. Wishart Invertida com $v$ gfaus de liberdade cuja densidade é dada por:

$$
f(W) \propto|W|^{-\frac{1}{2}(v+q+1)} \exp \left\{-\frac{1}{2} \operatorname{tr}\left|S W^{-1}\right|\right\}
$$

sendo $S$ é uma matriz escalàr quadradà $q \times q$, simétrica e definida positiva e $W$ é dëfinida positiva.

Para geramos amostras dā distribuiçãa postèriori conjuntả. (4.9), tëmos os seguinites passos:

i- Iniciar com valores inieiais $p_{1}^{(0)}, \mu_{1}^{(0)}, \mu_{2}^{(0)}, \Sigma_{1}^{(0)} \mathrm{e}-\Sigma_{2}^{(0)}$;

ii- Gerar uma amostra $\mathrm{Z}_{1}^{(1)}, \ldots, \mathrm{Z}_{n}^{(1)}$ da distribuição binomial com probabilidade de sucesso $v_{i j} \cdot(4.5)$.

iii- Gerar uma amostra de $\boldsymbol{\mu}_{1}, \boldsymbol{\mu}_{2}, \boldsymbol{\Sigma}_{1}$ and $\boldsymbol{\Sigma}_{2}$ das distribuiçōes condicionais dadas acima.

\subsubsection{Desenvolvimento do modelo com distribuição a priori con- jugada}

Cömo trabalhamos com fámílias exponenciais, propomos também um estudō com distribuições a priori conjugadas apresentado abaixo:

$$
\begin{gathered}
\pi(\theta)=\left|\Sigma_{1}\right|^{-\left(\frac{\left(g_{1}+a\right)}{2}+1\right)} \exp \left\{-\frac{1}{2} \operatorname{tr}\left[\mathbf{G}_{1} \Sigma_{1}^{-1} I-\frac{k_{1}}{2}\left(\mu_{1}-m_{1}\right)^{r} \Sigma_{1}^{-1}\left(\mu_{1}-m_{1}\right)\right\}\left|\Sigma_{2}\right|^{-\left(\frac{\left(g_{2}+a\right)}{2}+1\right)}\right. \\
\exp \left\{\frac{-1}{-} \operatorname{tr}\left[G_{2} \Sigma_{2}^{-1}\right]-\frac{k_{2}}{2}\left(\mu_{2}-m_{2}\right)^{\prime} \Sigma_{2}^{-1}\left(\mu_{2}-m_{2}\right)\right\}
\end{gathered}
$$

onde $\mathrm{G}_{\boldsymbol{i}}$ são matrizes de constantes conhecidas para cada sub-população, $\mathrm{m}_{\boldsymbol{i}}$ vetores de constantes conhecidas para cada sub-população e $g_{i}$ e $k_{i}$ parâmetros conhecidós para cada sub-população, ou seja, $i=1,2$. 


$$
\pi\left(p_{1}\right)=B \bar{e} t a(a, a, b) \text { onde } a, b \text { conhecidos. }
$$

Assumindo independênciā a priori, temos que a distribibição a posteriori será :

$$
\pi\left(\boldsymbol{\theta}, p_{1} \mid \mathrm{x}, \mathrm{z}\right) \propto \pi(\boldsymbol{\theta}) \pi\left(p_{1}\right) p_{1}^{\sum_{i=1}^{n} z_{i 1}} p_{2}^{\sum_{i=1}^{n} z_{i 2}} \prod_{i=1}^{n}\left\{\left[f\left(\mathrm{x}_{i} \mid \boldsymbol{\theta}_{1}\right)\right]^{z_{i 1}}\left[f\left(\mathrm{x}_{i} \mid \boldsymbol{\theta}_{2}\right)\right]^{z_{i 2}}\right\}
$$

onde $p_{2}=1-p_{1}, r=\sum_{i=1}^{n} z_{i 1}$ e. $n-r=\sum_{i=1}^{n} z_{i 2}$.

\subsubsection{Distribuições Condicionais a Posteriori Necessárias para 0}

\section{Algoritmo Gibbs. Sampling}

$$
\pi\left(\mathbf{p}_{1} \mid \boldsymbol{\theta}_{1} ; \theta_{2}, \mathrm{X}, \mathrm{z}\right) \propto \propto p_{1}^{(r+a)-1}\left(1-p_{1}\right)^{(n-r+b)-1}
$$

Portanto,

$$
\left(\mathrm{p}_{1} \mid \theta_{1}, \theta_{2}, \mathrm{x}, \mathrm{z}\right) \sim \operatorname{Beta}(r+a ; n-r+b)
$$

Para $\mu_{1}$ temos:

$$
\begin{aligned}
& \pi\left(\mu_{1} \mid \Sigma_{1}, p_{1}, \theta_{2}, \mathrm{x}, \mathrm{z}\right) \propto \exp \left\{-\frac{k_{ \pm}}{2}\left(\mu_{1}-\mathrm{m}_{1}\right)^{\prime} \boldsymbol{\Sigma}_{1}^{-1}\left(\mu_{1}-\mathrm{m}_{1}\right)\right\} \prod_{i=1}^{n}\left[\left[\left.\Sigma_{1}\right|^{-\frac{1}{2}} \exp \left\{-\frac{1}{2}\left(\mathrm{x}_{i}-\mu_{1}\right)^{\prime} \boldsymbol{\Sigma}_{1}^{-1}\left(\mathrm{x}_{i}-\mu_{1}\right)\right\}\right]^{z_{i 1}}\right. \\
& \propto \exp \left\{-\frac{k_{1}}{2}\left(\mu_{1}-\mathbf{m}_{1}\right)^{\prime} \boldsymbol{\Sigma}_{1}^{-1}\left(\mu_{1}-\mathbf{m}_{1}\right)\right\} \exp \left\{-\frac{1}{2} \sum_{i=1}^{n} z_{i 1}\left(x_{i}-\mu_{1}\right)^{\prime} \Sigma_{1}^{-1}\left(x_{i}-\mu_{1}\right)\right\} \\
& \propto \exp \left\{-\frac{k_{1}}{2}\left(\mu_{1}-\mathbf{m}_{1}\right)^{\prime} \bar{\Sigma}_{1}^{-1}\left(\mu_{1}-\mathbf{m}_{1}\right)\right\} \exp \left\{-\frac{r_{1}}{2}\left(\mu_{1}-\overline{\mathbf{x}}_{1}\right)^{\prime} \boldsymbol{\Sigma}_{1}^{-1}\left(\mu_{1}-\overline{\mathbf{x}}_{1}\right)\right\} \\
& \exp \left\{-\frac{1}{2} \operatorname{tr}\left[\Sigma_{1}^{-1} \mathbf{V}_{1}\right]\right\}
\end{aligned}
$$

onde $r_{1}=\sum_{i=1}^{n} z_{i 1} ; \quad \bar{x}_{1}=\frac{1}{r_{1}} \sum_{i=1}^{n} z_{i 1} x_{i} ; \quad V_{i}=\sum_{i=1}^{n} z_{i 1}\left(x_{i}-\bar{x}_{1}\right)\left(x_{i}-\bar{x}_{1}\right)^{\prime}$.

$$
\begin{aligned}
\pi\left(\mu_{1} \mid \Sigma_{1}, p_{1}, \theta_{2}, \mathbf{x}, \mathrm{z}\right) & \propto \exp \left\{-\frac{k_{1}}{2}\left(\mu_{1}-\mathbf{m}_{1}\right)^{\prime} \Sigma_{1}^{-1}\left(\mu_{1}-\mathbf{m}_{1}\right)\right\} \exp \left\{-\frac{r_{1}}{2}\left(\mu_{1}-\overline{\mathbf{x}}_{1}\right)^{\prime} \Sigma_{1}^{-1}\left(\mu_{1}-\overline{\mathbf{x}}_{1}\right)\right\} \\
& \propto \exp \left\{-\frac{1}{2}\left(\mu_{1}-\overline{\mathbf{x}}_{1}\right)^{\prime}\left(\frac{\Sigma_{1}}{r_{1}}\right)^{-1-}\left(\mu_{1}-\overline{\mathbf{x}}_{1}\right)+\left(\mu_{1}-\mathbf{m}_{1}\right)^{\prime}\left(\frac{\Sigma_{1}}{k_{1}}\right)^{-1}\left(\mu_{1}-\mathbf{m}_{1}\right)\right\} \\
& \propto \exp \left\{-\frac{1}{2}\left[\left(\mu_{1}-\mathbf{a}_{1}\right)^{\prime}\left(\frac{\boldsymbol{\Sigma}_{1}}{r_{1}+k_{1}}\right)^{-1}\left(\mu_{1}-a_{1}\right)+\left(\bar{x}_{1}-\mathbf{m}_{1}\right)^{\prime}\left(\frac{k_{1} r_{1}}{k_{1}+r_{1}}\right) \Sigma_{1}^{-1}\left(\overline{\mathbf{x}}_{1}-\mathbf{m}_{1}\right)\right]\right\} \\
& \propto \exp \left\{-\frac{1}{2}\left[\left(\mu_{1}-a_{1}\right)^{\prime}\left(\frac{\Sigma_{1}}{r_{1}+k_{1}}\right)^{-1}\left(\mu_{1}-a_{1}\right)\right\}\right.
\end{aligned}
$$


onde $a_{1}=\frac{r}{r_{1}+k_{1}} \bar{x}+\frac{k_{1}}{r_{1}+k_{1}} m_{1}$.

Portanto,

$$
\left(\boldsymbol{\mu}_{1} \mid \boldsymbol{\Sigma}_{1}, p_{1}, \boldsymbol{\theta}_{2}, \mathrm{x}, \mathrm{z}\right) \sim N_{\mathrm{q}}\left(\mathrm{a}_{1} ; \frac{\boldsymbol{\Sigma}_{1}}{r_{\mathrm{I}}+k_{1}}\right)
$$

Para $\boldsymbol{\Sigma}_{1}$ temos,

$$
\begin{aligned}
& \pi\left(\boldsymbol{\Sigma}_{1} \mid \boldsymbol{\mu}_{1}, \mathbf{p}_{1}, \boldsymbol{\theta}_{2}, \mathbf{x}, \mathbf{z}\right) \propto \int \pi\left(\boldsymbol{\theta}, \mathrm{p}_{1} \mid \mathbf{x}, z\right) d \boldsymbol{\mu}_{1} \\
& \propto\left|\Sigma_{1}\right|_{-}^{-\left(\frac{\left(g_{1}+q+1\right)}{2}\right)} \exp \left\{-\frac{1}{2} \operatorname{tr}\left[\mathbf{G}_{1} \Sigma_{1}^{-T} T\right\}\left|\Sigma_{1}\right|^{-\left(\frac{\left.r_{1}\right)}{2}\right.} \exp \left\{-\frac{1}{2} \operatorname{tr}\left[\mathbf{V}_{1} \Sigma_{1}^{-1}\right]\right\}\right\} \int\left|\Sigma_{1}\right|^{-\frac{1}{2}} \\
& \exp \left\{-\frac{1}{2}\left(\mu_{1}-\overline{\mathbf{x}}_{1}\right)^{\prime}\left(\frac{\mathbf{\Sigma}_{1}}{r_{1}}\right)^{-1}\left(\mu_{1}-\overline{\mathbf{x}}_{1}\right)+\left(\mu_{1}-\mathrm{m}_{1}\right)^{\prime}\left(\frac{\mathbf{\Sigma}_{1}}{k_{1}}\right)^{-1}\left(\mu_{1}-\mathrm{m}_{1}\right)\right\} d \mu_{1}
\end{aligned}
$$

Assim vamos ter,

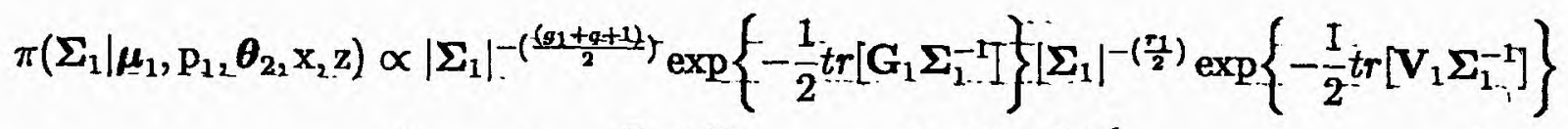

$$
\begin{aligned}
& \int\left|\Sigma_{1}\right|^{-\frac{1}{2}} \exp \left\{-\frac{1}{2}\left[\left(\mu_{1}-a_{1}\right)^{\prime} \cdot\left(\frac{\Sigma_{1}}{r_{1}+k_{1}}\right)^{-1}\left(\mu_{1}-a_{1}\right)+\left(\bar{x}_{1}-m_{1}\right)^{\prime}\right.\right. \\
& \left.\left.\left(\frac{k_{1} r_{1}}{k_{1}+r_{1}}\right) \underline{\Sigma}_{1}^{-1}\left(\overline{\mathbf{x}}_{1}-\mathrm{m}_{1}\right)\right]\right\} d \mu_{1} \\
& \propto\left|\Sigma_{1}\right|^{-\left(\frac{\left(g_{1}+q+1\right)}{2}\right)} \exp \left\{-\frac{1}{2} \operatorname{tr}\left[G_{1} \Sigma_{1}^{-1} T\right\}\left[\left.\Sigma_{1}\right|^{-\left(\frac{r_{1}}{2}\right)} \exp \left\{-\frac{1}{2} \operatorname{tr}\left[V_{1} \Sigma_{1}^{-i}\right]\right\}\right.\right. \\
& \exp \left\{-\frac{1}{2} \operatorname{tr}\left[\ddot{\Sigma}_{-1}\left(\frac{-k_{1} r_{1}}{k_{1}+r_{1}}\right)\left(\bar{x}_{1}-m_{1}\right)\left(\overline{\mathbf{x}}_{1}-\mathrm{m}_{1}\right)^{\prime}\right]\right\}
\end{aligned}
$$

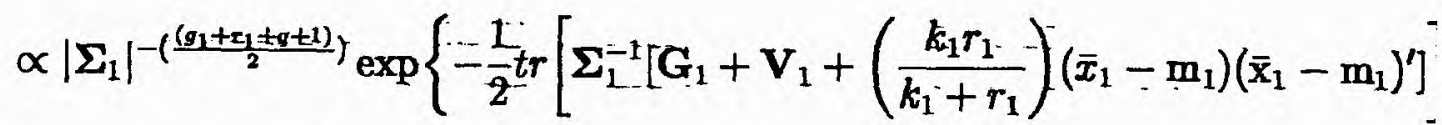

onde $\cdot V_{1}=\sum_{i=1}^{n} z_{i 1}\left(x_{i}-\bar{x}_{1}\right)\left(x_{i}-\bar{x}_{1}\right)^{r}$ e- $a_{1}=\frac{r_{i}}{r_{1}+k_{1}} \bar{x}+\frac{k_{\pi}}{r_{1}+k_{1}} m_{1}$.

Portainto,

$$
\left(\boldsymbol{\Sigma}_{1} \mid \boldsymbol{\mu}_{1}, \mathrm{p}_{1}, \boldsymbol{\theta}_{2}, \mathrm{x}, \mathrm{z}\right) \sim \operatorname{In} v-\text { Wishart } t_{\left(g_{1}+r_{1}\right)}\left(\mathbf{G}_{n}^{-1}\right)
$$

onde $\bar{G}_{n L}^{-1}=G_{1}+V_{1}+\left(\frac{k_{i} r_{1}}{k_{1}+r_{1}}\right)\left(\overline{\mathbf{x}}_{1}-m_{1}\right)\left(\overline{\mathbf{x}}_{1}-m_{1}\right)^{\prime}$

Da mesma forma,

$$
\left(\mu_{2} \mid \Sigma_{2}, p_{1}, \theta_{1}, \mathrm{x}, \mathrm{z}\right) \sim N_{q}\left(\mathrm{a}_{2} ; \frac{\boldsymbol{\Sigma}_{2}}{r_{2}+k_{2}}\right)
$$


onde

$$
\begin{aligned}
& \mathrm{a}_{2}=\frac{r_{2}}{r_{2}+k_{2}} \overline{\mathrm{x}}_{2}+\frac{k_{2}}{r_{2}+\bar{k}_{2}} \mathrm{~m}_{22} \quad \overline{\mathrm{x}}_{2}=\frac{1}{r_{2}} \sum_{i=1}^{n} z_{i 2} \mathrm{x}_{i,} \quad r_{2}=\sum_{i=1}^{n} z_{i 2} .
\end{aligned}
$$

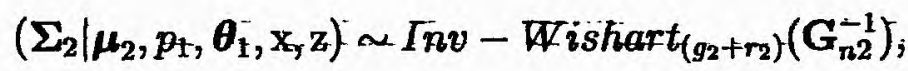

onde $\mathbf{G}_{n 2}^{-1}=\mathbf{G}_{2}+\mathbf{V}_{2}+\left(\frac{-k_{2} r_{2}}{k_{2}+r_{2}}\right)\left(\overline{\mathbf{x}}_{2}-\mathbf{m}_{2}\right)\left(\overline{\mathbf{x}}_{2}-\mathbf{m}_{2}\right)^{\prime}$.

Rèsultados similares podem ser obtidos para $K>2$.

\subsubsection{Classificação para duas Populações}

Nưm caso específico, vamos classificar um novo objeto em uma das duas populações

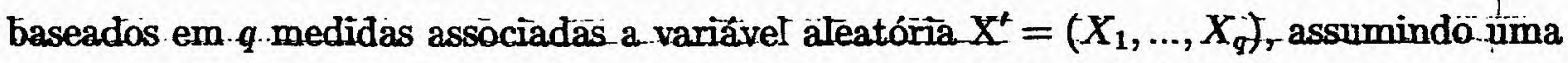
mistura de distribuiȩões normais $f^{(1)}\left(x \mid \theta^{(1)}\right)=\sum_{j=1}^{2} p_{j}^{(1)} f_{j}^{(1)}\left(x \mid \theta_{j}^{(1)}\right)$ para a população-1 e $f^{(2)}\left(\mathbf{x} \mid \theta^{(2)}\right)=\sum_{j=1}^{2-} p_{j}^{(2)} f_{j}^{(2)}\left(\bar{x} \mid \theta_{j}^{(2)}\right)$ para a população 2 ; onde $\theta_{j}^{(l)}=\left(\mu_{j}^{(l)}, \Sigma_{j}^{(l)}\right) \mathrm{e} \cdot f_{j}^{(l)}\left(\mathbf{x} \mid \dot{\theta}_{j}^{(l)}\right)$ denota uma distribuição normat multivariada $N_{g}\left(\mu_{j}^{(l)} ; \Sigma_{j}^{(t)}\right) ; j=1,2 ; \cdots t=1,2$.

A dēnsidadè preditiva párā o vetor $\mathbf{x}$ é dađơ por,

$$
f^{(l)}(\overline{\mathbf{x}})=\int \because f^{(l)}\left(\mathbf{x} \mid \theta^{(l)}\right) \pi\left(\theta^{(l)} \mid \mathbf{x}\right) d \theta^{(l)}
$$

onde $l=1$ ou 2 ( $(l$ indíces dās populāções 1 e 2 ).

Um estimador de Monte Cärlō-para $f^{(l)}(\bar{x})$ baseadö nas-amostras geradas pelo algoritmo de Gibbs é dado por,

$$
\hat{f}^{(l)}(\mathrm{x})=\frac{1}{S} \sum_{s=1}^{s} f^{(l)}\left(\mathrm{x} \mid \theta^{(l) s}\right)_{r}
$$

onde $S$ é o número de amostras geradas pelo Gibbs.

Para classificar um novo objeto com base em suas medidas observadās $\mathbf{x}$, cönsideramos a seguinte regra de classificação:

i- Alöcar x para a populäção 1 se

$$
\frac{\hat{f}^{(1)}(x)}{\hat{f}^{(2)}(x)} \geq\left[\frac{\bar{e}(1[2)}{c(2 \mid 1)}\right]\left[\frac{p_{2}}{p_{1}}\right] .
$$

onde $c(1 \mid 2)$ è $c(2 \mid 1)$ são os custos dèclassificaçãò errada; e $p_{1}$ e $p_{2}$ são as probąbilidades a priori de classificação para ambas populações, citados nö capítulo 2.

ii- Alocar x para população 2, caso contrário. 
Quando $c(1 \mid 2)=c(2 \mid 1)$ e $p_{1}=p_{2}$ a regra de classificação. é dada por:

i- Alocar x para a população 1 se,

$$
\frac{\hat{f}^{(1)}(\mathbf{x})}{\hat{f}^{(2)}(\mathbf{x})} \geq 1
$$

ii- Alocar x para a população 2, caso contrário.

\subsection{Um Exemplo Mustrativo}

Neste exemplo ilustrativo, geramos dùas amostras para cada populăçãò, de tamanho 100, com uma mistura de duas distribuições Normais Multivariadas, com os respectivos parâmetros para ambas as populäções:

Pop1:

$$
\begin{gathered}
\mu_{1}=\left(\begin{array}{ll}
2.5 & 4.5
\end{array}\right) ; \quad \boldsymbol{\Sigma}_{\mathrm{i}}=\left(\begin{array}{cc}
1 & 0.3 \\
0.3 & 1.5
\end{array}\right) ; \quad p_{1}=0.4 \\
\mu_{2}=\left(\begin{array}{ll}
4.0 & 10.0
\end{array}\right) ; \quad \boldsymbol{\Sigma}_{2}=\left(\begin{array}{cc}
2.0 & 0.4 \\
0.4 & -2.5
\end{array}\right) ; \quad p_{2}=0.6
\end{gathered}
$$

Pop2:

$$
\begin{gathered}
\mu_{1}=\left(\begin{array}{ll}
3.5 & 5.5
\end{array}\right) ; \quad \Sigma_{1}=\left(\begin{array}{cc}
1.0 & 0.3 \\
0.3 & 2.0
\end{array}\right) ; \quad p_{1}=0.5 \\
\mu_{2}=\left(\begin{array}{ll}
6.5 & 14.6
\end{array}\right) ; \quad \Sigma_{2}=\left(\begin{array}{ll}
2.0 & 0.4 \\
0.4 & 3.0
\end{array}\right) ; p_{2}=0.5
\end{gathered}
$$

Os gráficos para os dois conjuntos são dados pela Figura 4.1 


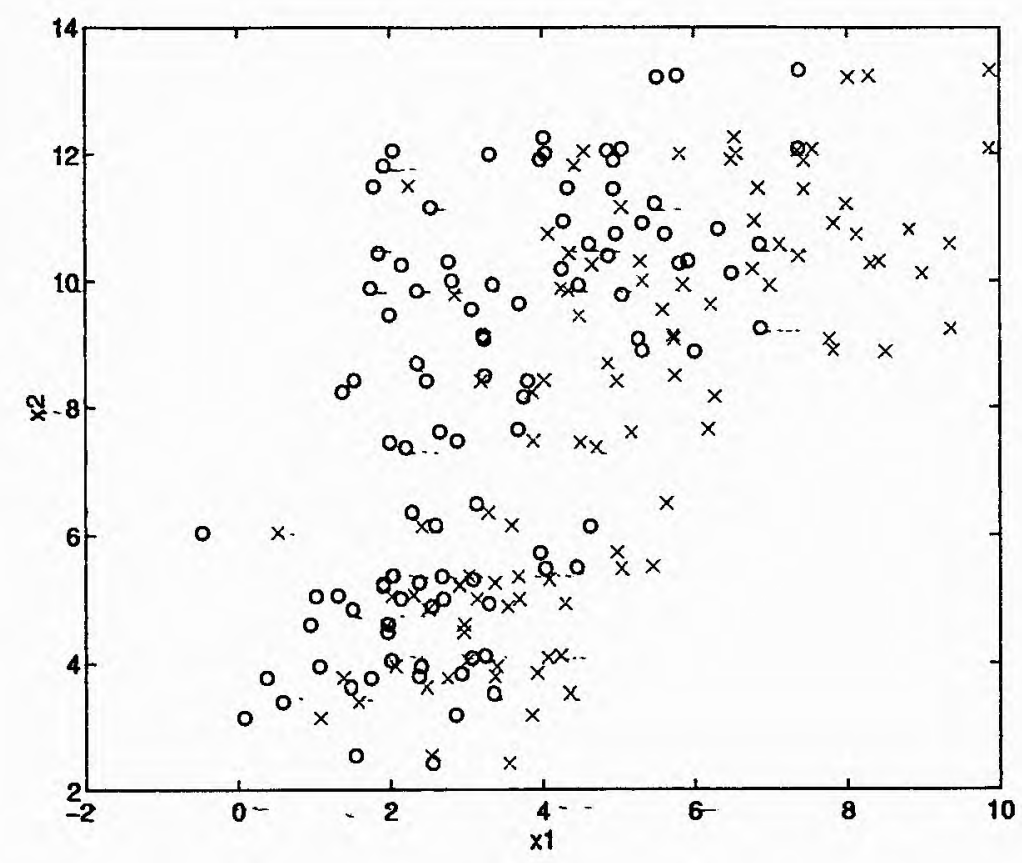

Eigura 4.1: Dàdos das duas Popiulaçōes: pop1 (o), pop2( $(x)$.

Percebemos nestè conjuntó de dados, um comportàmento caracterizadó por uma mistura de duas sub-populações. Os dados se mostram concentrados em duas regiōes distintas. Neste caso, verificamos que os modelōs̄ tradicìionais de classificaçãò mostram-se pouco eficientes.

\subsubsection{Utilização dos.Procedimentos Usuais de Classificação}

Aquui vamos utilizàr os dois procedimentos de classificação mais usuais, já discutidos anteriormentë: a Função Discriminante Liñear(E.L.) e a Funçãa Discriminạante Quadrática.(F.D.Q.).

Utilizando a F:D.L., chègamos a seguinte Tabela-de classificação:

Tabela 4.1:Tabela de Classificação Mèmbro pelo Critériò Discriminanante

\begin{tabular}{c|c|cccc|c}
\hline & & $\pi_{1}$ & taxa & $\pi_{2}$ & taxa & valor real \\
\hline Membro & $\pi_{1}$ & 70 & 70.00 & 30 & 30.00 & 100 \\
Verdadeiro & $\pi_{2}$ & 40 & 40.00 & 60 & 60.00 & 100 \\
\hline
\end{tabular}


Assim temos,

$$
A P E R=\frac{n_{1 M}+n_{2 M}}{n_{1}+\pi_{2}}=\frac{30+40}{100+100}=0.35
$$

onde $n_{i M}, i=1,2$ é o número de clàssificações erradàs para cada grupo ou seja, númeroro de elementos que são do grupo $i$ e foram classificados no grupo $k$ onde $i, k=1,2$ com $i \neq k$.

Proporção de Classificação correta:

$$
P r C=1-E C=0.65
$$

Utilizando a F.D.Q., chegamos a seguinte Tabela de classificação:

Tabela 4.2:Tabela de Cłassificação

\begin{tabular}{c|c|cccc|c}
\multicolumn{7}{c}{ Membro pelo Critério Discriminante } \\
\hline & & $\pi_{1}$ & taxa & $\pi_{2}$ & taxa & valor real \\
\hline Membro & $\pi_{1}$ & 76 & 76.00 & $24^{-}$ & 24.00 & 100 \\
Verdadeiro & $\pi_{2}$ & 41 & 41.00 & 59 & 59.00 & 100 \\
\hline
\end{tabular}

Assim temos,

$$
A P E R=\frac{24+41}{100+100}=0.325
$$

Proporção de Classificação correta:

$$
\operatorname{Pr} C=1-E C=0.675
$$

Com esses dois critérios de classificação utilizados, percebemos uma proporção total de classificação correta um pouco bäixa, devido a característica específica dōs dädos. Assim, verificamos que os procedimentos tradicionais não são muito eficientes quanto temos este tipo de dado, justificando a procura por alternativas que nos levem a melhores resultados.

\subsection{Aplicação dos Modelos de Mistura para a Clas- sificação}

Nesta seção utilizamos os procedìmentos de classificação considerando a densidade de cada população como uma mistura de duas distribuições Normais Multivariadas, com o intuíto de melhor modelarmos os dädōs, consequientemente proporcionar uma melhor 
taxa de classificação correta. Aqui apresentamos o enfoque Bayesiano para a solução do problema considerando dístribiuiçôes a priori não informativa e informativa para os parâmetros.

Para os procedimentos Bayesianos, fòram geradas 10000 amostras de Gibbs para cada parâmetro, sendo descartadas as 4000 amostras iniciais e depois tomadas as amostras restantes de 20 em 20, totalizando uma amostra final de 300 observaçõès para cada parâmetro. Dado o grande número de parâmetros envolvidos e a dificuldade computacional para realização do algoritmo multivariado, trabalhamos apenas com uma grande cadeia. O.critériö usado para a análiše dè convergência foi o de Geweke,J. (1992). Aqui, todo o procedimento de simulação foi gerada a partir do Software $O \dot{x}$ 2.10. (ver Doornik,1996).

\subsubsection{Utilização de. Distribuições a Priori Não Informativas}

Nesta análise consideramos uma mistiura de 2 dis̄tribuiçōes normaîs bivariadas $(4,1)$ $\operatorname{com} \theta_{1}^{(l)}=\left(\mu_{1}^{(l)}, \bar{\Sigma}_{1}^{(l)}\right)$ e $\quad \theta_{2}^{(l)}=\left(\mu_{2}^{(l)}, \Sigma_{2}^{(t)}\right)$ onde,

$$
\left.\boldsymbol{\mu}_{1}^{(l)}=\left(\mu_{11}^{(l)} ; \mu_{12}^{(l)}\right), \quad \mu_{2}^{(l)}=f \mu_{21}^{(l)} ; \mu_{22}^{(l)}\right), \quad \mathbf{\Sigma}_{1}^{(l)}=\left(\begin{array}{cc}
\ddot{\sigma}_{111}^{(l)} & \sigma_{112}^{(l)} \\
\sigma_{121}^{(l)} & \sigma_{122}^{(l)}
\end{array}\right), \quad \boldsymbol{\Sigma}_{2}^{(l)}=\left(\begin{array}{ll}
\dot{\sigma}_{211}^{(l)} & \sigma_{212}^{(l)} \\
\sigma_{221}^{(l)} & \sigma_{222}^{(l)}
\end{array}\right)
$$

para $l=1$ (Pop1) e $l=2$ (Pöp2) e.os parâmetros dà distribuição a priōin (4.8), com a e $b=3$ para Pop1 e $a=2$ e $b=2$ para Pop2.

Na Tabela 4.3 apresentamos um resumo as estimativas dos parâmetros das duas populações e a seguir os gráficos para a convergência e as dénsidadè marginais posteriores.

Table 4.3 - Resumos das Estimativas (Mistura de 2 Distribuições Normais Bivariadàs; Diștribuiçâo a Priōio (4.8) para $\theta$ )

\begin{tabular}{|cccccc|}
\hline & Parâmetro & Méđìa & S:D. & 95\% Intervalo de Confiança & $|G W|$ \\
\hline & $\mu_{11}^{(1)}$ & 2.2180 & 0.20796 & $(1.8231 ; 2.5952)$ & 0.0187 \\
\multirow{4}{*}{ Pop1 } & $\mu_{12}^{(1)}$ & 4.7413 & 0.36945 & $(4.1906 ; 5.6530)$ & 0.0312 \\
& $\sigma_{111}^{(1)}$ & 1.2288 & 0.33482 & $(0.70499 ; 2.0119)$ & 0.0256 \\
& $\sigma_{122}^{(1)}$ & 2.0556 & 1.1650 & $(0.79698 ; 5.2064)$ & 0.0104 \\
& $\sigma_{112}^{(1)}$ & 0.38458 & 0.32913 & $(-0.22956 ; 1.0659)$ & 0.0864 \\
& $p_{1}^{(1)}$ & 0.38848 & 0.069225 & $(0.26940 ; 0.54735)$ & 0.0455 \\
\hline
\end{tabular}


Table 4.3 - Continuação

\begin{tabular}{|cccccc|}
\hline & Parâmetro & Média & S.D. & $95 \%$ Intervalo de Confiança & $|G W|$ \\
\hline & $\mu_{21}^{(1)}$ & 4.0117 & 0.28724 & $(3.4918 ; 4.7025)$ & 0.0163 \\
& $\mu_{22}^{(1)}$ & 10.0520 & 0.40065 & $(9.1946 ; 10.722)$ & 0.0523 \\
& $\sigma_{211}^{(1)}$ & 2.5543 & 0.50716 & $(1.6696 ; 3.7523)$ & 0.0164 \\
& $\sigma_{222}^{(1)}$ & 4.1283 & 1.4710 & $(2.0013 ; 8.0003)$ & 0.0704 \\
& $\sigma_{212}^{(1)}$ & 1.1125 & 0.5469 & $(0.15604 ; 2.3419)$ & 0.0175 \\
\hline \multirow{4}{*}{ Pop2 } & $\mu_{11}^{(2)}$ & 3.1585 & 0.1700 & $(2.8474 ; 3.5063)$ & 0.0497 \\
& $\mu_{12}^{(2)}$ & 5.5554 & 0.18671 & $(5.1742 ; 5.9197)$ & 0.0539 \\
& $\sigma_{111}^{(2)}$ & 1.2615 & 0.28094 & $(0.82515 ; 1.9301)$ & 0.0025 \\
& $\sigma_{122}^{(2)}$ & 1.6764 & 0.43747 & $(1.0427 ; 2.5983)$ & 0.0131 \\
& $\sigma_{112}^{(2)}$ & 0.18441 & 0.2566 & $(-0.31451 ; 0.7831)$ & 0.0327 \\
& $p_{1}^{(2)}$ & 0.44199 & 0.046343 & $(0.3554 ; 0.52604)$ & 0.0075 \\
& $\mu_{21}^{(2)}$ & 6.5406 & 0.2432 & $(5.9708 ; 7.0236)$ & 0.0036 \\
& $\mu_{22}^{(2)}$ & 14.80 & 0.2556 & $(14.250 ; 15.305)$ & 0.0310 \\
& $\sigma_{21}^{(2)}$ & 2.4759 & 0.49652 & $(1.7055 ; 3.5964)$ & 0.0055 \\
& $\sigma_{222}^{(2)}$ & 4.4486 & 1.0596 & $(2.7541 ; 7.0163)$ & 0.0112 \\
& $\sigma_{212}^{(2)}$ & 1.2344 & 0.55629 & $(0.19226 ; 2.5609)$ & 0.0174 \\
\hline
\end{tabular}

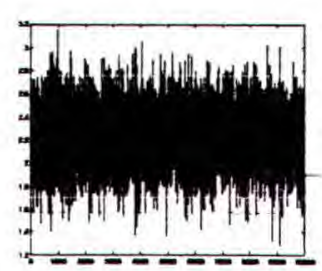

$\mu_{11}^{(1)}$

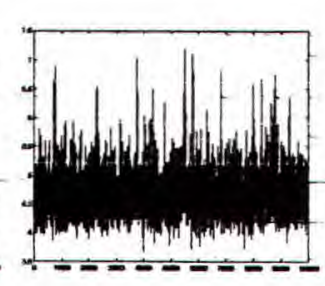

$\mu_{12}^{(1)}$

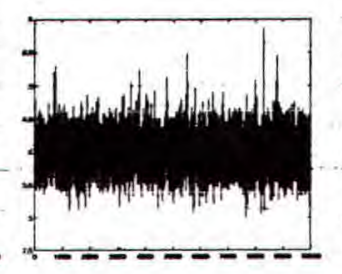

$\mu_{21}^{(1)}$

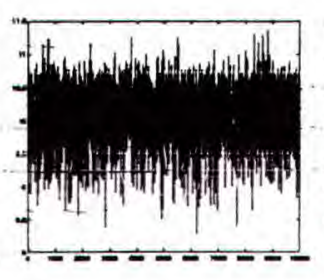

$\mu_{22}^{(1)}$

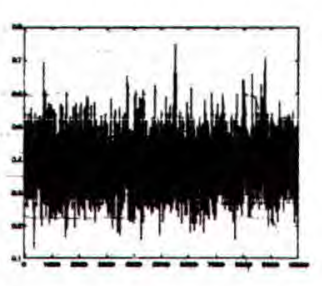

$p_{1}^{(1)}$

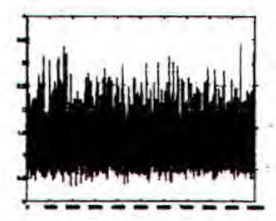

$\sigma_{111}^{(1)}$

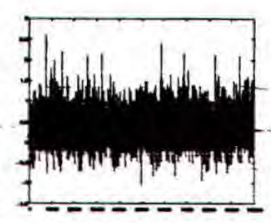

$\sigma_{122}^{(1)}$

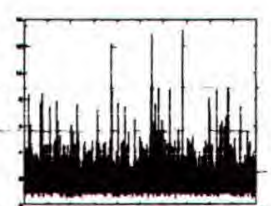

$\sigma_{112}^{(1)}$

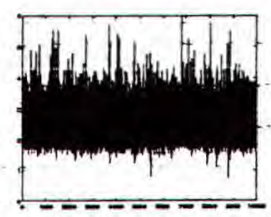

$\sigma_{211}^{(1)}$

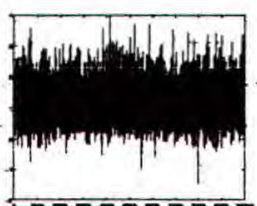

$\sigma_{222}^{(1)}$

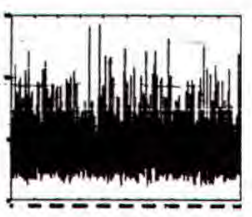

$\sigma_{212}^{(1)}$

Figura 4.2: Convergência dos parâmetros (Pop1). 

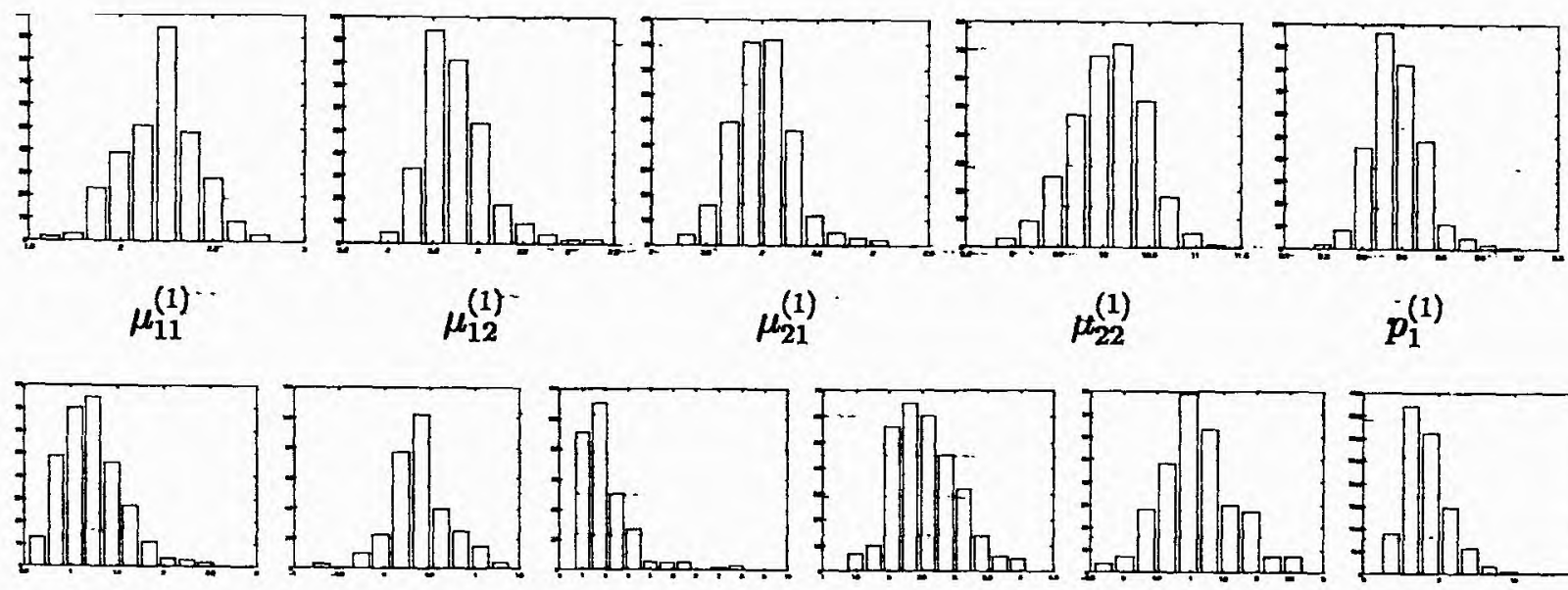

$\mu_{22}^{(1)}$

$\dot{p}_{1}^{(1)}$

$\sigma_{111}^{(1)}$

$\sigma_{122}^{(1)}$

$\sigma_{112}^{(1)}$
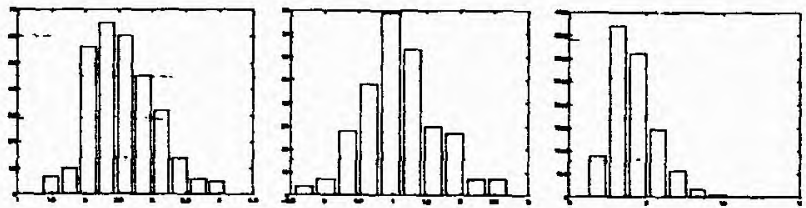

$\sigma_{211}^{(1)}$

$\sigma_{222}^{(1)}$

$\sigma_{212}^{(1)}$

Figura 4.3: Dènsidades Marginais a. Pösteriori (Pop1).

Abaixo tèmos os gráficos para a Pop2.

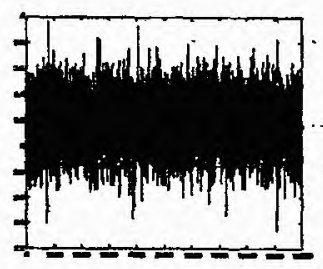

$\mu_{11}^{(2)}$

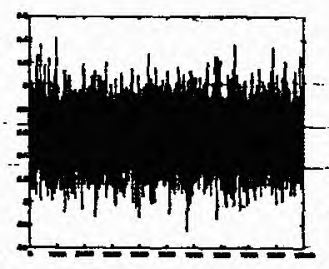

$\mu_{12}^{(2)}$

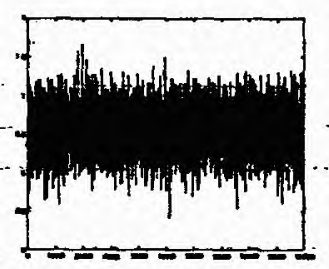

$\mu_{21}^{(2)}$

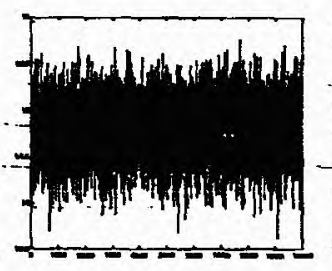

$\mu_{22}^{(2)}$

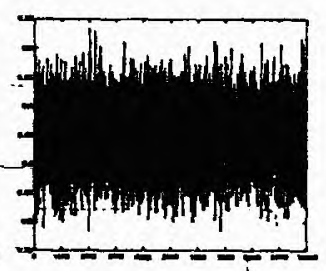

$-p_{1}^{(2)}$

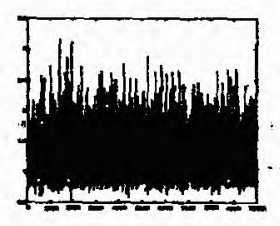

$\sigma_{111}^{(2)}$

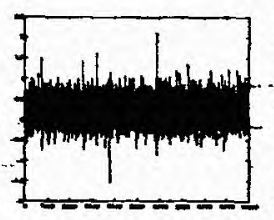

$\sigma_{122}^{(2)}$

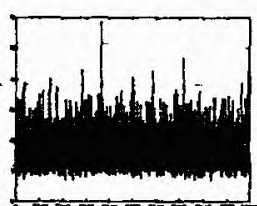

$\sigma_{112}^{(2)}$

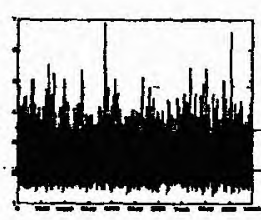

$\sigma_{211}^{(2)}$

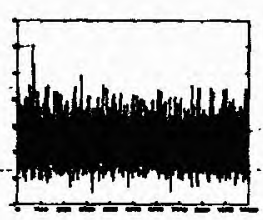

$\sigma_{222}^{(2)}$

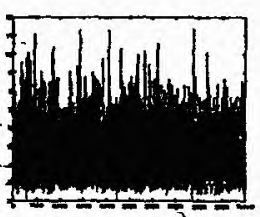

$\sigma_{212}^{(2)}$

Figura 4.4: Convergência dơs parâñnetros.(Pop2). 

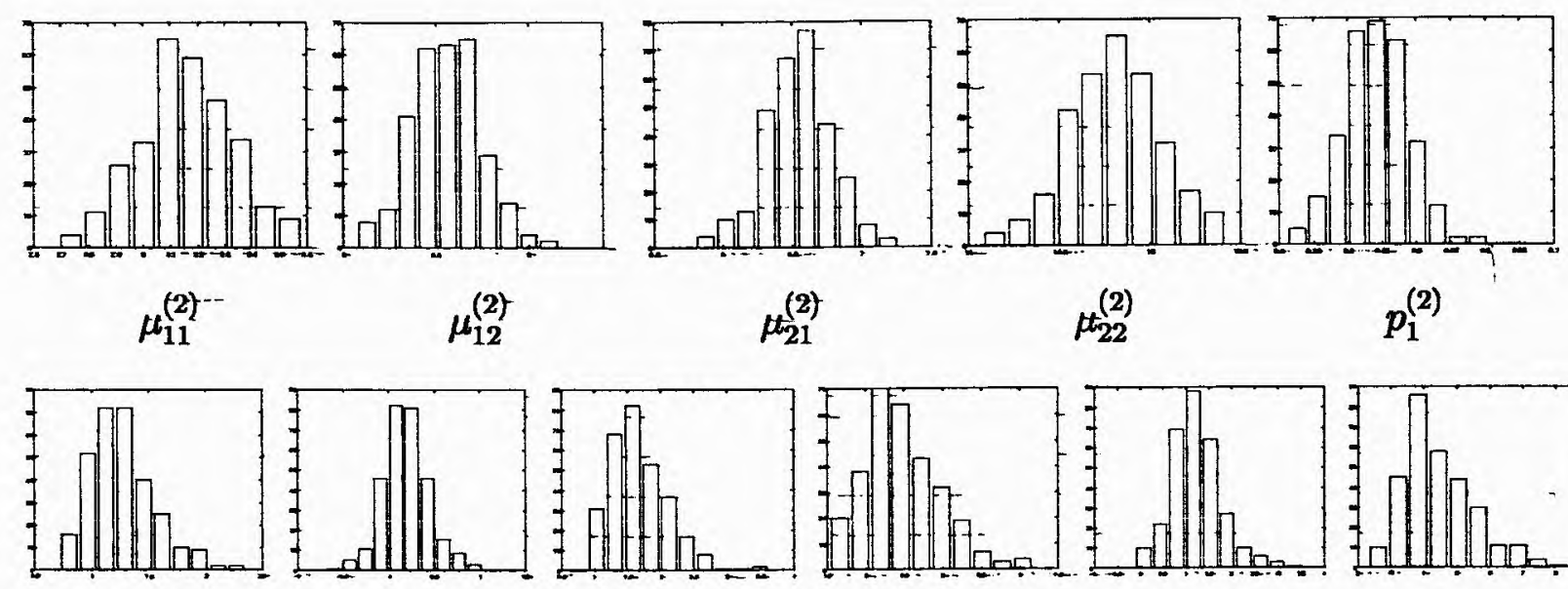

$\mu_{11}^{(2)-}$

$\mu_{12}^{(2)}$

$\mu_{21}^{(2)}$

$\mu_{22}^{(2)}$

$p_{1}^{(2)}$
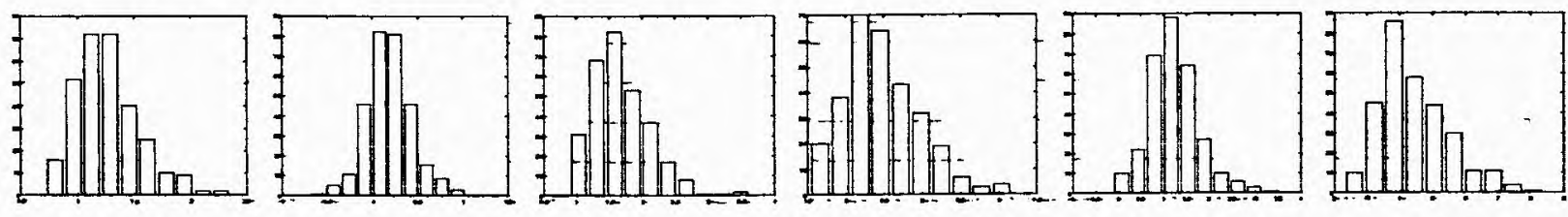

$\sigma_{111}^{(2)}$

$\sigma_{122}^{(2)}$

$\sigma_{112}^{(2)}$

$\sigma_{211}^{(2)}$

$\sigma_{222}^{(2)}$

$\sigma_{212}^{(2)}$

Figura 4.5:-Densidades Marginais a.Posteriori (Pop2).

Podemos notar que obtivemos bons resultados para a convergência, pois para todos os parâmetros temos $|G W|<2$, além dá constatação visual pelas Figuras 4.2 e. 4.4 .

Nà Tabēla 4.4-abaixo, temos a tabela de clàssificação.

Tabela 4.4: Tabela de Classificação

\begin{tabular}{c|c|cccc|c}
\multicolumn{7}{c}{ Membro pelo Critério Discriminante } \\
\hline & & $\pi_{1}$ & taxa & $\pi_{2}$ & taxa & valor real \\
\hline Membro & $\pi_{1}$ & 83 & 83.00 & 17 & 17.00 & 100 \\
Verdadeiro & $\pi_{2}$ & 19 & $19: 00$ & 81 & $81: 00$ & 100 \\
\hline
\end{tabular}

Assim temos,

$$
E C=\frac{17+19}{100+100}=0: 18
$$

Proporção de Classificação correta:

$$
\operatorname{Pr} C=\mathrm{t}-E C \doteq 0.82
$$

Considerando este modelo de mistura, percebemos um grande aumento na proporção total de classificação correta. Istoo mostra que o modelo proposto se adequa melhor aos dados, conseqüentemente melhorando a taxa de classificação.Verificamos agora o desempenho deste critério quando utiliżamos uma distribuição a priori informațiva. 


\subsubsection{Utilização de Distribuições a Priori Informativas}

Nèsta análise consideramos as distribuições a priori dadas em (4.15 e 4.J6)

Os parâmetros da distribuição a priori para as duas Populações são dados abaixo:

Pop1:

$$
\begin{gathered}
\mathrm{m}_{1}=\left(\begin{array}{ll}
2.5 & 4.5
\end{array}\right) ; \quad \mathbf{G}_{1}=\left(\begin{array}{cc}
\tau & 0.3 \\
0.3 & 1.5
\end{array}\right) ; \\
\mathrm{m}_{2}=\left(\begin{array}{cc}
4.0 & 10.0
\end{array}\right) ; \quad \mathrm{G}_{2}=\left(\begin{array}{cc}
2.0 & 0.4 \\
-0.4 & 2.5
\end{array}\right) \\
k_{1}=3 ; \quad k_{2}=3 ; \quad g_{1}=7 \quad g_{2}=7 ; \quad a=10 ; b=10 .
\end{gathered}
$$

Pop2:

$$
\begin{aligned}
& \mathrm{m}_{1}=\left(\begin{array}{ll}
3.5 & 5.5
\end{array}\right) ; \quad \mathbf{G}_{1}=\left(\begin{array}{cc}
1.0 & 0.3 \\
0.3 & 2.0
\end{array}\right) \\
& \mathrm{m}_{2}=(6.5-14.6) ; \quad \mathbf{G}_{2}=\left(\begin{array}{ll}
2.0 & 0.4 \\
0.4 & 3.0
\end{array}\right) ; \\
& k_{1}=3 ; \quad k_{2}=3 ; \quad g_{1}=7 ; \quad g_{2}=7 ; \quad a=10 ; b=10 .
\end{aligned}
$$

Os procedimentos de simulação foram semelhảntes aos usados no exemplo anterior, considerando agora as distribuições condicionais a posteriori dadàs na seção (4.3.4.4). Abaixo na Tabelà 4:5, apresentamos um resumo das estimativas dos parâmetros para as duas populações e a seguir os gráficos para a convergência e as densidades marginais a posteriores.

Tảelà 4.5 - Resumōs dàs Estìnativas (Mistura de 2 Distribuiç̧ôes Noripais Bivariadas; Distribuições a Priori (4.15) e (4.16)

\begin{tabular}{|cccccc|}
\hline & Parâmetro & Média & S.D: - & 95\% Imtervato de Confiança & $\mid$ GW \\
\hline & $\mu_{11-}^{(1)}$ & 2.2626 & 0.19234 & $(1.8744 ; 2.6433)$ & 0.0019 \\
& $\mu_{12}^{(1)}$ & 4.5904 & 0.22286 & $(4.2052 ; 5.0603)$ & 0.0116 \\
& $\sigma_{111}^{(1)}$ & 0.94689 & 0.2639 & $(0.55589 ; 1.6046)$ & 0.0736 \\
Pop1 1 & $\sigma_{122}^{(1)}$ & 1.3017 & 0.47161 & $(0.70885 ; 2.5620)$ & 0.0588 \\
& $\sigma_{112}^{(1)}$ & 0.3243 & 0.20275 & $(0.004668 ; 0.81366)$ & 0.0719 \\
& $p_{1}^{(1)}$ & 0.39912 & 0.052701 & $(0.29853 ; 0.50799)$ & 0.0189 \\
& $\mu_{21}^{(1)}$ & 3.9721 & 0.22622 & $(3.5397 ; 4.4167)$ & 0.0531 \\
\hline
\end{tabular}


Tabela 4.5 - Continuação

\begin{tabular}{|c|c|c|c|c|c|}
\hline & Parâmetro & Média & S.D:- & 95\% Intervato de Confiança & $|G W|$ \\
\hline \multirow{4}{*}{ Pop1 } & $\mu_{22}^{(1)}$ & 10.054 & 0.28728 & $(9.4443 ; 10.579)$ & 0.1254 \\
\hline & $\sigma_{211}^{(1)}$ & 2.233 & 0.39157 & $(1.616 ; 3.0994)$ & 0.0404 \\
\hline & $\sigma_{222}^{(1)}$ & 3.6273 & 0.95053 & $(2.0555 ; 5.8916)$ & 0.0801 \\
\hline & $\sigma_{212}^{(1)}$ & 0.99682 & 0.42114 & $(0.26592 ; 1.9683)$ & 0.0718 \\
\hline \multirow{11}{*}{ Pop2 } & $\mu_{11}^{(2)}$ & 3.2034 . & 0.16598 & . $(2.8589 ; 3.5076)$ & 0.0114 \\
\hline & $\mu_{12}^{(2)}$ & 5.6200 & 0.1778 & $(5.2761 ; 5.9473)$ & 0.0023 \\
\hline & $\sigma_{111}^{(2)}$ & $1.0981 \ldots$ & 0.2387 & $(0.71551 ; 1.5960)$ & 0.0348 \\
\hline & $\sigma_{122}^{(2)}$ & 1.5455 & 0.35837 & $(0.98285 ; 2.3600)$ & 0.0296 \\
\hline & $\sigma_{112}^{(2)}$ & 0.20325 & 0.19768 & $(-0.20371 ; 0.63515) \cdots$ & $\theta .0405$ \\
\hline & $p_{i}^{(2)}$ & 0.45774 & 0.044559 & $(0.37268 ; 0.53877)$ & 0.0274 \\
\hline & $\mu_{21}^{(2)}$ & 6.5791 & 0.20661 & $(6.1442 ; 6.9367)$ & $\theta .0154$ \\
\hline & $\mu_{22-}^{(2)}$ & 14.811 & 0.23635 & $(14.385 ; 15.286)$ & 0.0009 \\
\hline & $\sigma_{211}^{(2)}$ & $2.113 T^{\cdots}$ & 0.41496 & $(1.4666 ; 3.1241)$ & 0.0266 \\
\hline & $\sigma_{222}^{(2)}$ & 3.7948 & 0.72236 & $-(2.559 ; 5.3511)$ & 0.0649 \\
\hline & $\sigma_{212}^{(2)-}$ & 1.0023 & 0.41995 & $(0.26454 ; 1.8881)$ & 0.0542 \\
\hline
\end{tabular}

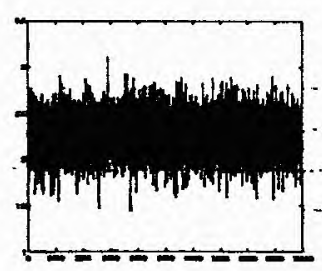

$\mu_{11}^{(1)}$

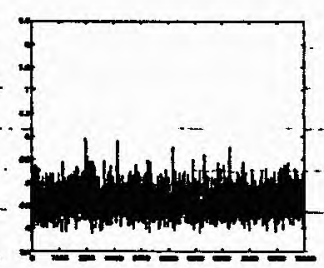

$\mu_{12}^{(1)^{-}}$

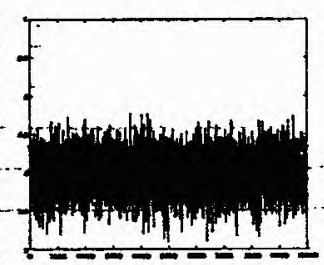

$\mu_{21}^{(1)}$

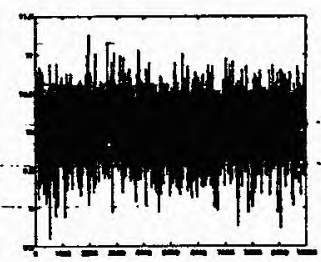

$\mu_{22}^{(1)}$

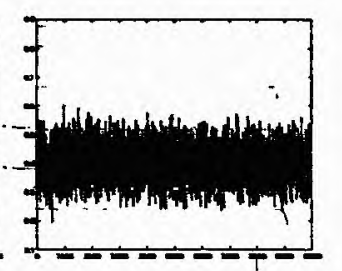

$p_{1}^{(1)}$

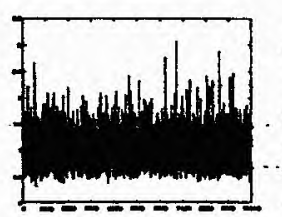

$\sigma_{111}^{(1)}$

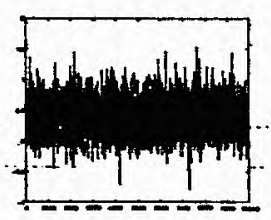

$\sigma_{122}^{(1)}$

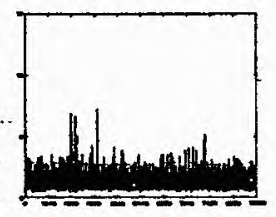

$\sigma_{112}^{(1)}$

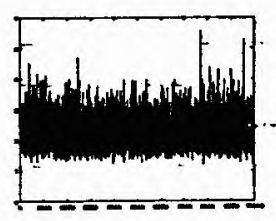

$\sigma_{211}^{(1)}$

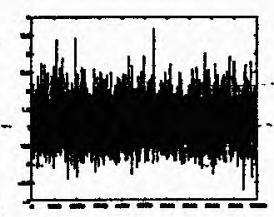

$\sigma_{222}^{(1)}$

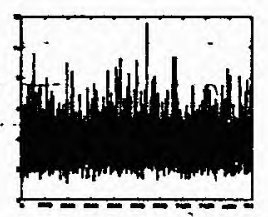

$\sigma_{212}^{(1)}$

Figura 4.6: Convergênīia dös parâmetros (Pop1). 


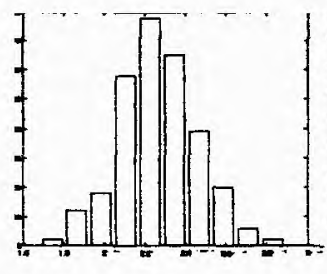

$\mu_{11}^{(1)}$

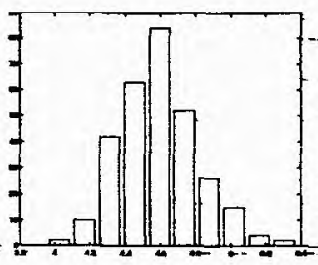

$\mu_{12}^{(1)}$

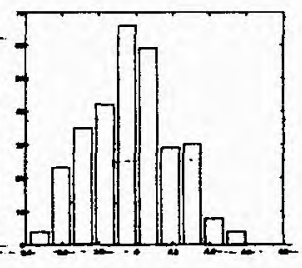

$\mu_{21}^{(1)-}$

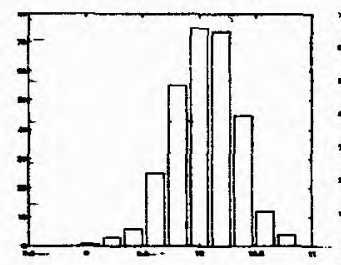

$\mu_{22}^{(1)}$

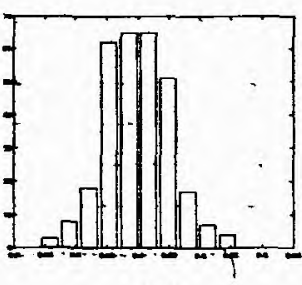

$\dot{p}_{1}^{(1)}$

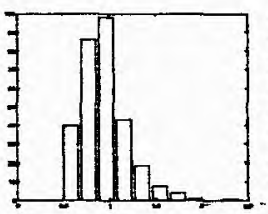

$\sigma_{111}^{(1)}$

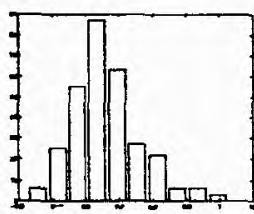

$\sigma_{122}^{(1)}$

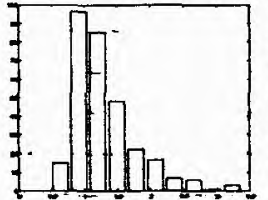

$\sigma_{112}^{(1)}$

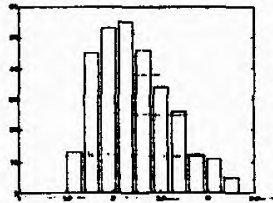

$\sigma_{211}^{(1)}$

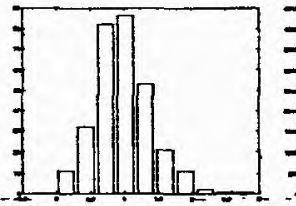

$\sigma_{222}^{(1)}$

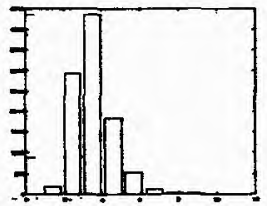

$\sigma_{212}^{(1)}$

Figura 4.7: Densidades Marginais a Posteriori (Pop1).

Abaixo temos os gráficos para a Pop2.

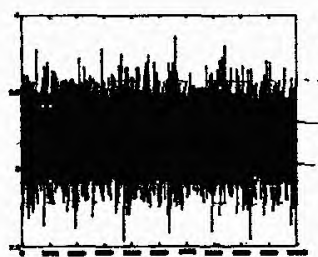

$\mu_{11}^{(2)}$

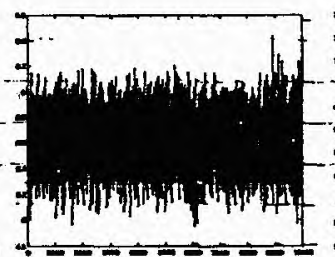

$\mu_{12}^{(2)}$

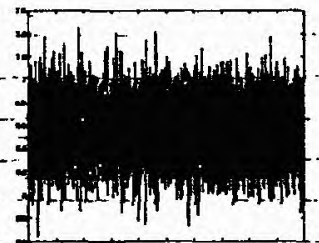

$\mu_{21}^{(2)}$

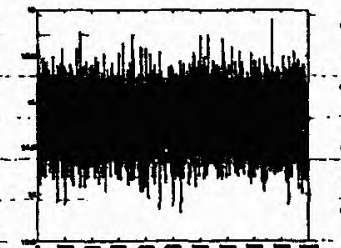

$\mathrm{H}_{22}^{(2)}$

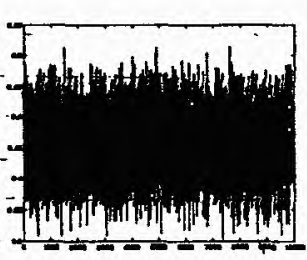

$-p_{1}^{(2)}$
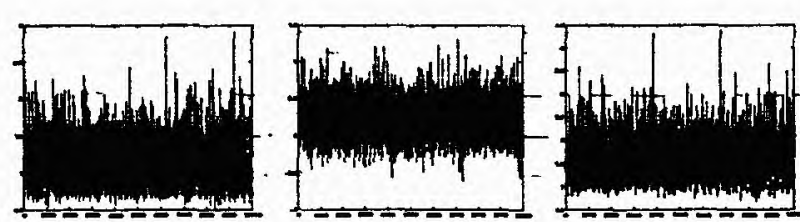

$\sigma_{122}^{(2)}$

$\sigma_{112}^{(2)}$
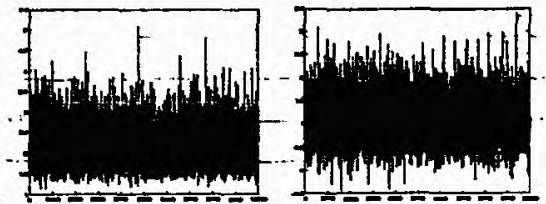

$\sigma_{211}^{(2)}$

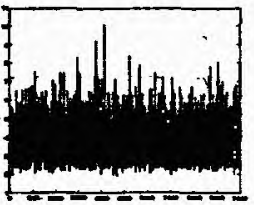

$\sigma_{222}^{(2)}$

$\sigma_{212}^{(2)}$

Figura 4.8:-Convergência dos parâmetros (Pop2). 

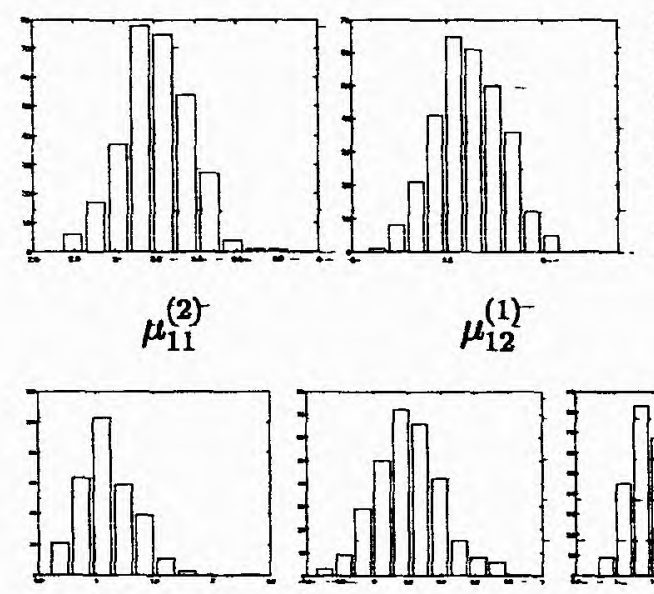

$\sigma_{111}^{(2)}$

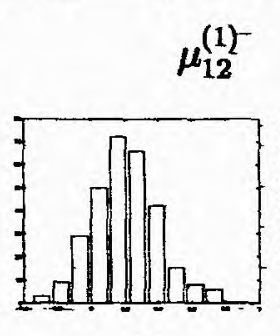

$\sigma_{122}^{(2)}$

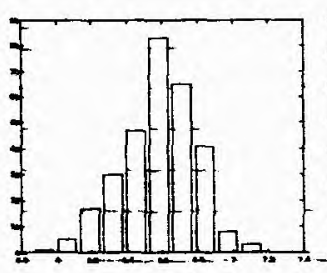

$\mu_{21}^{(2)}$

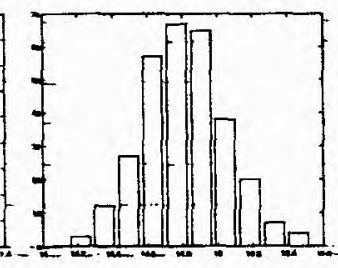

$t_{22}^{(2)}$

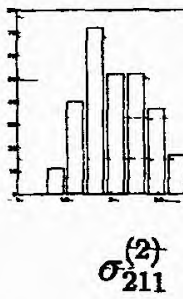

$\sigma_{222}^{(2)}$

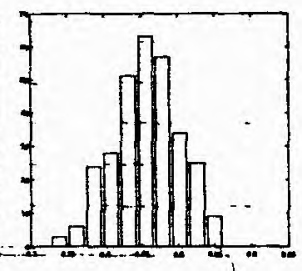

$p_{1}^{(2)}$
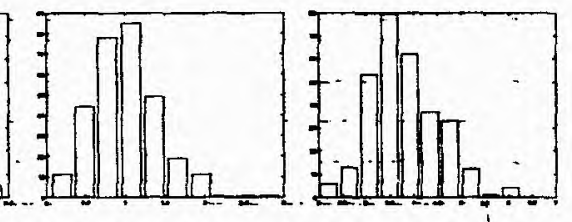

$\sigma_{212}^{(2)}$

Figura 4:9: Deñsidades Marginais a Posteriori (Pop2).

Novamente percebemos bons resultados para a convergência. Abaixo temos a Tabela de classificação.

Tàbela 4.6:Tàbela de Clàssificação

\begin{tabular}{c|c|cccc|c}
\multicolumn{7}{c}{ Membro pelo Critério Discriminante } \\
\hline & & $\pi_{1}$ & taxa & $\pi_{2}$ & taxa & valor real \\
\hline Mèmbro & $\pi_{1}$ & $86^{-}$ & 86.00 & $14 \cdots$ & $14.00 \cdots$ & 100 \\
Verdadeiro & $\pi_{2}$ & $18^{-}$ & 18.00 & $82 \cdots$ & 82.00 & 100 \\
\hline
\end{tabular}

Assim temos,

$$
E C=\frac{14+18}{10 \theta+100}=0.16
$$

Proporção de Classificação cơrreta:

$$
\operatorname{Pr} C \cong 1-E C=0: 84
$$

Para a distribuição a priori informativa, conseguimos um considerável aumento na proporção total de clássificação correta, isto se dá ao fato de termos uma excelente informação a priori. Abaixo, temos a classificação, quando utilizamos o algoritmo EM.

\subsubsection{Classificação Utilizando o algoritmo EM}

Nesta análise consideramos a estimação dos parâmetros de cada função de densidade, pelo algoritmo EM (ver McLachlan \& Krishnan, 1997) e usamos as densidades estimadas 
na regra de classificação para cada observação.

As condições iniciais necessárias para o algoritmo EM, dadas abaixo, foram escolhịdas com base em várias simulações,

Os parâmetros da distribuição a priori para as duas Populações são dados abaixo:

Pop1:

$$
\begin{gathered}
\mathrm{m}_{1}=\left(\begin{array}{ll}
2.5 & 4.5
\end{array}\right) ; \quad \mathrm{G}_{1}=\left(\begin{array}{cc}
\mathrm{I} & 0.3 \\
0.3 & 1.5
\end{array}\right) \\
\mathrm{m}_{2}=\left(\begin{array}{cc}
4.0 & 10.0
\end{array}\right) ; \quad \mathrm{G}_{2}=\left(\begin{array}{cc}
2.0 & 0.4 \\
0.4 & 2.5
\end{array}\right) ; \\
\left.p_{1}=0.4 \ldots \text { (e) }\right) . \quad p_{2}=0.6 .
\end{gathered}
$$

Pop2:

$$
\begin{gathered}
\mathrm{m}_{1}=\left(\begin{array}{ll}
3.5 & 5.5
\end{array}\right) ; \quad \mathrm{G}_{1}=\left(\begin{array}{cc}
1.0 & 0.3 \\
0.3 & 2.0
\end{array}\right) ; \\
\mathrm{m}_{2}=\left(\begin{array}{ll}
6.5 & 14.6
\end{array}\right) ; \quad \mathrm{G}_{2}=\left(\begin{array}{ll}
2.0 & 0.4 \\
0.4 & 3.0
\end{array}\right) ; \\
p_{1}=0.5 \quad \mathrm{e} p_{2}=0.5 .
\end{gathered}
$$

Abaixo temos as estimativas pontuais para as respectivas populações.

Tabela 4.7:Estimativas Pontuais: EM

\begin{tabular}{|c|cccccc|}
\hline \multirow{4}{*}{ Pop1 } & $\mu_{11}^{(1)}$ & $\mu_{12}^{(1)}$ & $\sigma_{111}^{(1)}$ & $\sigma_{112}^{(1)}$ & $\sigma_{122}^{(1)}$ & $p_{1}^{(1)}$ \\
\cline { 2 - 7 } & 2.3395 & 4.5873 & 1.0910 & 0.46699 & 1.0267 & 0.38 \\
\cline { 2 - 7 } & $\mu_{21}^{(1)}$ & $\mu_{22}^{(1)}$ & $\sigma_{211}^{(1)}$ & $\sigma_{212}^{(1)}$ & $\sigma_{222}^{(1)}$ & $p_{2}^{(1)}$ \\
& 3.9138 & 10.073 & 2.9161 & 1.4646 & 2.9391 & 0.62 \\
\hline \hline \multirow{3}{*}{ Pop2 } & $\mu_{11}^{(2)}$ & $\mu_{12}^{(2)}$ & $\sigma_{111}^{(2)}$ & $\sigma_{112}^{(2)}$ & $\sigma_{122}^{(2)}$ & $p_{1}^{(2)}$ \\
& 3.1775 & 5.58 & 1.0576 & 0.1936 & 1.4385 & 0.45 \\
\cline { 2 - 7 } & $\mu_{21}^{(2)}$ & $\mu_{22}^{(2)}$ & $\sigma_{211}^{(2)}$ & $\sigma_{212}^{(2)}$ & $\sigma_{222}^{(2)}$ & $p_{2}^{(2)}$ \\
& 6.5746 & $14: 842$ & 2.6099 & 1.2188 & 3.0831 & 0.55 \\
\hline
\end{tabular}

Abaixo temos a Tabela de classificação: 
Tabela 4.8:Tabela de Classificação

Membro pelo Critério - Discriminante

\begin{tabular}{c|c|cccc|c}
\hline & & $\pi_{1}$ & taxa & $\pi_{2}$ & taxa & valor real \\
\hline Mémbro & $\pi_{1}$ & 84 & $0.84^{-}$ & $14^{-}$ & 0.16 & 100 \\
Verdadeiro & $\pi_{2}$ & 18 & 0.18 & 82 & 0.82 & 100 \\
\hline
\end{tabular}

Assim temos,

$$
E C=\frac{16+18}{100+100}=0.17
$$

Proporção de Classificação correta:

$$
\operatorname{Pr} C=1-E C=0.83
$$

Considerando a distribuição a priori informativa, conseguimos um considerável aumento na proporção total dē classificação correta. Isto mostra que o modèto proposto modela melhor os dados, e como o aproveitamento da informação a priori leva a melhores resultados. Neste caso, considerando dados gerados, tívemos bons resultados, pois temos boas informações a priori. Talvez, em situações mais reais não tenhamos tanto sucesso, e uma soluçãa clássica (uso do EM) ou a consideração dãnão informação seja mais indiçado.

Um fato importante é que foi verificada a ineficácia dos procedimentos tradicionais de classificação quando trabalhamos com este tipo de dados. 


\section{Capítulo 5}

\section{Classificação e Discriminação a partir de Variáveis Binárias}

Neste capítulo apresentamos o problema de classificação quando os componentes do vetor aleatório $\mathrm{X}$ de medidas referente ao objeto são variáveis binárias, ou seja, assumem um de dois possíveis valores (sucesso $=1$ ou fracasso=0). Em várias áreas de interesse percebemos tais fatos. Por exemplo, na área médica podemos discriminar um paciente em estado grave ou não com base em medidas binárias do tipo :

- Tem problema renal ( $\operatorname{sim}=1$, não=0).

- Infecção adquirida na UTI (sim=1, não=0).

- Presença de Câncer ( $\operatorname{sim}=1$, não=0).

- Sexo $(E=1, M=0)$.

Em geral; existem vários outros métodos que podem ser aplicados ao problema de classificação quando temos este tipo de variável, como por exemplo, a Regressão Logística (ver por exemplo Hosmer \& Lemeshow, 1989) ou a métoda discriminante apresentado par Krzanowsk(1975), onde é proposto a procedimento de classificaçãa com misturas de variáveis contínuas e binárias. Neste trabalho apresentamos o modelo discriminante com base no procedimento geral de classificação descrito no Capítulo 2 e incorporamos procedimentos Bayesianos, utilizando novamente simulações via MCMC, formulando assim um procedimento alternativo. 


\subsection{Desenvolvimento do Critério de Classificação}

Da mesma forma descrita anteriormente, nos concentramos na classificação para duas populações embora os resultados possam ser generalizados para mais de duas populações.

Supor que é dado um vetor aleatório binário de medidas $\mathrm{X}$ associado a um abjeto(pessoa) de cada uma de duas populações. Assumindo inicialmente variáveis binạ́rias independentes, temos que a densidade para cada população é dada por,

$$
\begin{aligned}
& f_{1}(\mathrm{x})=\prod_{k=1}^{q} p_{1 k}^{x_{h}}\left(1-p_{1 k}\right)^{1-x_{k}}, \text { para Pop } 1 ; \\
& f_{2}(\mathrm{x})=\prod_{k=1}^{q} p_{2 k}^{x_{k}}\left(1-p_{2 k}\right)^{1-x_{k}}, \text { para Pop2; }
\end{aligned}
$$

onde $\mathrm{x}=\left(x_{1}, \ldots, x_{q}\right) ; x_{k}=(0$ ou 1$) ; k=1, \ldots, q$.

Fazendo a razão teremos,

$$
\frac{f_{1}(\mathrm{x})}{f_{2}(\mathrm{x})}=\frac{\prod_{k=1}^{q} p_{1 k}^{x_{k}}\left(1-p_{\uparrow k}\right)^{\mathrm{t}-x_{k}}}{\prod_{k=1}^{q} p_{2 k}^{x_{k}}\left(1-p_{2 k}\right)^{1-x_{k}}}=\left\{\frac{\prod_{k=1}^{q}\left(1-p_{1 k}\right)}{\prod_{k=1}^{q}\left(1-p_{2 k}\right)}\right\}\left\{\prod_{k=1}^{q}\left(\frac{p_{1 k}\left(1-p_{2 k}\right)}{p_{2 k}\left(1-p_{1 k}\right)}\right)^{x_{k}}\right\}
$$

Aplicando o logaritmo,

$$
\log \left\{\frac{f_{1}(\mathrm{x})}{f_{2}(\mathrm{x})}\right\}=\log \left\{\prod_{k=1}^{q}\left(\frac{\left.1-p_{1 k}\right)}{\left.1-p_{2 k}\right)}\right)\right\}+\sum_{k=1}^{q} x_{k} \log \left\{\frac{p_{1 k}\left(1-p_{2 k}\right)}{p_{2 k}\left(1-p_{1 k}\right)}\right\}
$$

Definir:

$$
\begin{aligned}
& B_{0}=\log \left\{\prod_{k=1}^{q}\left(\frac{\left.1-p_{1 k}\right)}{\left.1-p_{2 k}\right)}\right)\right\} \\
& \mathrm{e} \\
& B_{k}=\log \left\{\frac{p_{1 k}\left(1-p_{2 k}\right)}{p_{2 k}\left(1-p_{1 k}\right)}\right\}
\end{aligned}
$$

para $k=1, \ldots, q$.

Assim, baseado na regra de classificação geral descrita no capítulo 2, considerando custos de classificação errada e probabilidades a priori para cada população iguais, temos a seguinte regra de alocação,

i- Alocar $\mathrm{x}=\left(x_{1}, \ldots, x_{q}\right)$ à população $1 \mathrm{se}$,

$$
B_{0}+\sum_{k=1}^{q} B_{k} x_{k}>0
$$

ii- Alocar x para população 2, caso contrário. 
Considerando probabilidades a priori para os dois grupos temos,

i- Alacar $\mathrm{x}=\left(x_{1}, \ldots, x_{q}\right)$ à população 1 se,

$$
B_{0}+\sum_{k=1}^{q} B_{k} x_{k}>\log \left(\frac{\pi_{2}}{\pi_{1}}\right)
$$

ii- Alocar x para população 2, caso contrário,

onde $\pi_{1}$ é a probabilidade a priori de classificação na população 1 e $\pi_{2}$ é a probabilidade a priori de classificação na população 2 .

Os estimadores para $p_{j k} ; j=1,2 ; k=1, \ldots, q$ são calculados, tomando para a população $1 n_{1}$ observações e para a população $2 n_{2}$, com $n=n_{1}+n_{2}$ : tamanho total da amostra preliminar. Assim temos,

$$
\begin{aligned}
& \hat{p}_{1 k}=\frac{\sum_{i=1}^{n_{1}} x_{k i}}{n_{1}} \\
& \hat{p}_{2 k}=\frac{\sum_{i=1}^{n_{2}} x_{k i}}{n_{2}}
\end{aligned}
$$

onde $i=1, \ldots, n_{j}, j=1,2$.

Daí, temos,

$$
\begin{aligned}
& \hat{B}_{0}=\log \left\{\prod_{k=1}^{q}\left(\frac{\left.1-\hat{p}_{1 k}\right)}{\left.1-\hat{p}_{2 k}\right)}\right)\right\} \\
& \mathrm{e} \\
& \hat{B}_{k}=\log \left\{\frac{\hat{p}_{1 k}\left(1-\hat{p}_{2 k}\right)}{\hat{p}_{2 k}\left(1-\hat{p}_{1 k}\right)}\right\}
\end{aligned}
$$

para $k=1, \ldots, q$.

Portanto, uma regra de classificação amostral simples é dada por,

i- Alocar $\mathrm{x}=\left(x_{1}, \ldots, x_{q}\right)$ à população 1 se,

$$
\hat{B}_{0}+\sum_{k=1}^{q} \hat{B}_{k} x_{k}>0
$$

ii- Alocar x para população 2, caso contrário.

Na próxima seção será apresentado uma alternativa Bayesiana para o problema. 


\subsection{Alternativa Bayesiana para a Classificação-Binária.}

Nesta seção apresentamos uma alternativa Bayesiana para a classificação com dạdos binários, utilizando métodos MCMC, em especial o algoritmo Gibbs Sampling, usando estimativas de Monte Carlo para as densidades preditivas que são utilizadas na regra de classificação. Considerando a população 1, a verossimilhança para o modelo é dada por,

$$
L\left(\mathrm{p}_{1}\right)=L\left(p_{11}, \ldots, p_{1 q}\right)=\prod_{i=1}^{n_{r}} \prod_{k=1}^{q} p_{1 k}^{x_{k i}}\left(1-p_{1 k}\right)^{1-x_{k i}}
$$

Da mesma forma para a população 2 temos,

$$
L\left(\mathrm{p}_{2}\right)=E\left(p_{21}, \ldots, p_{2 q}\right)=\prod_{i=1}^{n_{2}} \prod_{k=1}^{q} p_{2 k}^{x_{k i}}\left(1-p_{2 k}\right)^{1-x_{k i}},
$$

onde $\mathrm{p}_{j}=\left(p_{j 1}, \ldots, p_{j q}\right)$ e $j=1,2$.

Fazendo para cada grupo,

$$
\begin{aligned}
& \bar{x}_{k}^{(1)}=\frac{1}{n_{1}} \sum_{i=1}^{n_{1}} x_{k i} \text {, para a pop.1. } \\
& \bar{x}_{k}^{(2)}=\frac{1}{n_{2}} \sum_{i=1}^{n_{2}} x_{k i} \text {, para a pop.2. }
\end{aligned}
$$

temos,

$$
\begin{aligned}
& L\left(\mathrm{p}_{1}\right)=\prod_{k=1}^{q} p_{1 k}^{n_{1} \bar{x}_{k}^{(1)}}\left(1-p_{1 k}\right)^{n_{1} \bar{x}_{k}^{(1)}} \text { para a pop.1. } \\
& L\left(\mathrm{p}_{2}\right)=\prod_{k=1}^{q} p_{2 k}^{n_{2} \bar{x}_{k}^{(2)}}\left(1-p_{2 k}\right)^{n_{2} \bar{x}_{k}^{(2)}} \text { para a pop.2. }
\end{aligned}
$$

Consideramos aqui distribuições a priori dadas por,

$$
\begin{aligned}
& p_{1 k} \sim \operatorname{Beta}\left(a_{1 k}, b_{1 k}\right) \\
& p_{2 k} \sim \operatorname{Beta}\left(a_{2 k}, b_{2 k}\right),
\end{aligned}
$$

para $k=1, \ldots, q$, onde $\operatorname{Beta}(a, b)$ denota uma distribuição Beta com média $\frac{a}{a+b}$ e variância $\frac{a b}{(a+b)^{2}(a+b+1)}$. 
Considerando as distribuições a priori independentes e combinando com as expressões dadas em.(5.11), temos as distribuç̧ões a posteriori conjunta para cada população dạdas por,

$$
\begin{aligned}
& \pi\left(p_{1} \mid D_{1}\right) \propto \prod_{k=1}^{q} p_{1 k}^{a_{1 k}+n_{1} \bar{x}_{k}^{(t)}}\left(1-p_{1 k}\right)^{b_{1 k}+n_{1} \bar{x}_{k}^{(1)}} \text { para a pop.1 } \\
& \pi\left(p_{2} \mid D_{2}\right) \propto \prod_{k=1}^{q} p_{2 k}^{a_{2 k}+n_{2} \bar{x}_{k-}^{(2)}}\left(1-p_{2 k}\right)^{b_{2 k}+n_{2} \bar{x}_{k}^{(2)}} \text { para a pop.2, }
\end{aligned}
$$

onde $D_{j}, j=1,2 ;$ representa as observações de cada grupo.

Assim, as distribuições condicionais necessárias para o algoritmo Gibbs Sampling para cada população são dados abaixo.

Pāra Pop1 temos,

$$
\left(p_{1 k} \mid D_{1}\right) \sim \operatorname{Beta}\left(a_{1 k}+n_{1} \bar{x}_{k}^{(1)}, b_{1 k}+n_{1}-\bar{x}_{k}^{(1)}\right)
$$

Para Pop2 temos,

$$
\left(p_{2 k} \mid D_{2}\right) \sim B e t a\left(a_{2 k}+n_{1} \bar{x}_{k}^{(2)}, b_{2 k}+n_{2}-\bar{x}_{k}^{(2)}\right)
$$

Asssim, a densidade preditiva para cadã população será dã forma,

$$
\begin{aligned}
& f_{1}(x)=f_{\theta_{1}} f_{1}\left(\dot{x} \mid p_{1}\right) \pi\left(p_{1} \mid D_{1}\right) d p_{1} \\
& f_{2}(x)=f_{\Theta_{2}} f_{2}\left(x \mid p_{2}\right) \pi\left(p_{2} \mid D_{2}\right) d p_{2}
\end{aligned}
$$

onde $\theta_{j}, j=1,2$; é o espaço de parâmetros da $j$-ésima população.

Gerado as amostras de $p_{1}$ e $p_{2}$ a partir do algoritmo Gibbs Sampling, usamos as estimativas de Mönte Carlōpara $f_{\mathrm{H}}(\mathrm{x})$ è $f_{2}(\mathrm{x})$ dadas poror,

$$
\begin{aligned}
& \hat{f}_{1}(\mathrm{x})=\frac{1}{s} \sum_{s=1}^{S} f_{1}\left(\mathrm{x} \mid \mathrm{p}_{\mathrm{I}}^{(s)}\right) \\
& \hat{f}_{2}(\mathrm{x})=\frac{1}{S} \sum_{s=1}^{S} f_{2}\left(\mathrm{x} \mid \mathrm{p}_{2}^{(3)}\right)
\end{aligned}
$$


onde $f_{j}\left(\mathrm{x} \mid \mathrm{p}_{j}\right)=\mathrm{H}_{k=1}^{q} p_{j k}^{x_{k}}\left(1=p_{j k}\right)^{1-x_{k}, \ldots, c o m} \mathrm{x}=\left(x_{1}, \ldots, x_{q}\right) ;$ e. $S$ o númenero de amoștras geradas pelo algoritmo. Portapto,

$$
\begin{aligned}
& \hat{f}_{1}(\mathrm{x})=\frac{1}{s} \sum_{s=1}^{S}\left\{\prod_{k=1}^{q-1}\left(p_{1 k}^{(o)}\right)^{x_{k}}\left(1-p_{1 k}^{(\diamond)}\right)^{1-x_{k}}\right\} \\
& \hat{f}_{2}(\mathrm{x})=\frac{1}{S} \sum_{s=1}^{S}\left\{\prod_{k=1}^{q-1}\left(p_{2 k}^{(o)}\right)^{x_{k}}\left(1-p_{2 k}^{(s)}\right)^{1-x_{k}}\right\}
\end{aligned}
$$

Novamente a regra de classificação é dada por,

i- Alocar $\mathrm{x}=\left(\dot{x}_{1}, \ldots, x_{q}\right)$ à̀ populăção I se,

$$
\frac{\hat{f}_{1}(\mathrm{x})}{\hat{f}_{1}(\mathrm{x})}>1
$$

ii- Alocar x para população 2, caso contrário.

\section{3 . Exemplo de-Aplicação.}

Vámos ilustrar um problèma de classificação com dados binários a partir dos dados de Hösmer(ver Hösmer,D.W.;Lèmeshiōw,S;1989). São dađōos de internação numa. UTII dẹ ùm hospital. Pära os pacientes internadós. na UTI (200 pacientes ao total), há dois grupos:

a). Pacientes que sobreviyem.

b) Pacientes_que não sobrevivem.

Pàia cada paciente, existe uma série de variáveis binárias associadās. Abaixo vamos descrever as variáveis usadas neste exemplo.

1- $x_{1}=$ Idade $(\operatorname{anos})(\geq 60=1 ;<60=0)$;

2- $x_{2}=$ Sexxo $(\mathrm{F}=1 ; \mathrm{M}=0)$.

$3-x_{3}=$ Raça $($ branco $=1 ;$ não branco $=0)$.

4- $x_{4}=$ Tìpo de admissão à UTI.(médico $=0$; cirurgia $=1$ ).

5- $x_{5}=$ Presença de câncer. $($ sim $=1 ;$ não $=0)$. 
6- $x_{6}=$ Problema renal $(\operatorname{sim}=1 ; n a \tilde{a}=0)$.

7- $x_{7}=$ Infeç̧ão adquiridāna UTI( $\operatorname{sim}=1 ;$ กล̃o $\left.=0\right)$.

8- $x_{8}=$ cpr prévia à admissão na U'TItsim $=1 ;$ não $=0$ ).

$9 x_{9}=$ Pressão sistólica no momento dà admissãona UTI $(\geq 150=1 ;<150=0)$.

10- $x_{10}=$ Batidas do coraçãa no momenta da internaçãa na UTI $(>110=1 ; \leq 110 ; 0)$.

11- $x_{11}=$ Iñternação prévia $(6$ meses anteriores $)(\operatorname{sim}=1 ;$ não $=0)$.

12- $x_{12}=$ Tipo de internação (èmergência $=1$; elètiva $\left.=0\right)$.

13- $x_{13}=$ Presença de fratura $($ sim $=1 ; n a ̃ o=0)$.

$14-x_{14}=P o_{2}$ no sangue $(\leqq 60=I ;>60=0)$.

15- $x_{15}=\mathrm{PH}$ no sangue $(<7.25=1 ; \geq 7.25 \equiv 0)$.

$16-x_{16}=E C O_{2}$ no sangue $(>45=1 ; \leq 45=0)$.

17: $x_{17}=$ Bicarbonato no sangue $(<18=1 ; \geqq 18=0)$.

Assumimos imicialmente variáveis binárias independentes para os dois grupos. Com base nestas variáveis vamos classificar as pessoas em sobreviventes ou não sobreviventes. Para este conjunto de dados temos 17 variáveis ou seja $k=1, \ldots, 17$, para o grupo de sobreviventes, temos $n_{1}=160$ observações e para os não sobreviventes temos $n_{2}=40$, totalizando assim uma amostra prévia de $n=n_{1}+n_{2}=200$ observações.

Primeiro utilizamos o procedimento geral de classificação. Abaixo, na Tabela 6.1, temos as estimativas para os parâmetros $\mathrm{p}_{j k}$, estimados com base no procedimento apresentado na seção 6.1 .

Table 6.1- Estimativas para $\mathrm{p}_{j k}$

\begin{tabular}{|c|ccccccccc|}
\hline & $p_{j 1}$ & $p_{j 2}$ & $p_{j 3}$ & $p_{j 4}$ & $p_{j 5}$ & $p_{j 6}$ & $p_{j 7}$ & $p_{j 8}$ & $p_{j 9}$ \\
Pop1 & 0.537 & 0.375 & 0.862 & 0.581 & 0.100 & 0.0687 & 0.375 & 0.0375 & 0.300 \\
Pop2 & 0.725 & 0.400 & 0.925 & 0.350 & 0.100 & 0.200 & 0.600 & 0.175 & 0.125 \\
\hline \hline & $p_{j 10}$ & $p_{j 11}$ & $p_{j 12}$ & $p_{j 13}$ & $p_{j 14}$ & $p_{j 15}$ & $p_{j 16}$ & $p_{j 17}$ & - \\
Pop1 & 0.318 & 0.143 & 0.681 & 0.056 & 0.068 & 0.056 & 0.100 & 0.062 & - \\
Pop2 & 0.350 & 0.175 & 0.950 & 0.100 & 0.125 & 0.100 & 0.100 & 0.125 & - \\
\hline
\end{tabular}


Abaixo temos a Tabela 6.2, tabela de classificação,

Tabela 6.2:Tabela de Classificação

Pela Função Discriminante

\begin{tabular}{c|c|cccc|c}
\hline & & Pōp1 & taxa & Pōp2 & taxa & valor real \\
\hline Membro & Pop1 & 109 & 68.10 & 51 & 31.80 & 160 \\
Verdadeiro & Pop2- & 11 & 27.50 & 29 & 72.50 & 40 \\
\hline
\end{tabular}

Assim a taxa de erro aparente $(A P E R)$ é igual a:

$$
A P E R=\frac{n_{1 M} \pm n_{2 M}}{n_{1}+n_{2}}=\frac{51+11}{160+40}=0.31
$$

Os resultados da classificação para este tipo de dado parecem não ser muito eficientes. No entanto, dão ao pesquisador uma idéia da situação preliminar de um novo paciente.

Agora vamos checar a eficiência do procedim̄nento Bayesiano.

Abaixo, na Tabela 6.3, temos os parâmetros escolhidos' a priori para $a_{j k}$ e $b_{j k, j} j=$ 1,$2 ; k=1, \ldots, 17$.

Table 6.3-Parâmetros a Priori

\begin{tabular}{|c|cccc|}
\hline & $a_{1 k}$ & $b_{1 k}$ & $a_{2 k}$ & $b_{2 k}$ \\
\hline$k=1$ & 0.1 & 0.09 & 0.1 & 0.04 \\
$k=2$ & 0.1 & 0.17 & 0.10 & 0.15 \\
$k=3$ & 0.1 & 0.02 & 0.10 & 0.04 \\
$k=4$ & 0.1 & 0.07 & 0.1 & 0.18 \\
$k=5$ & 0.1 & 0.9 & 0.1 & -0.9 \\
$k=6$ & 0.1 & 1.32 & 0.1 & .04 \\
$k=7$ & 0.1 & 0.17 & 0.10 & 0.067 \\
$k=8$ & 0.1 & 2.4 & 0.10 & 0.49 \\
$k=9$ & 0.1 & 0.23 & 0.10 & 0.73 \\
$k=10$ & 0.1 & 0.22 & 0.10 & 0.18 \\
$k=11$ & 0.1 & 0.61 & 0.10 & 0.49 \\
$k=12$ & 0.1 & 0.05 & 0.10 & 0.095 \\
$k=13$ & 0.1 & 1.68 & 0.10 & 0.90 \\
$k=17$ & $0.1 \cdots$ & $1.37 \cdots$ & 0.10 & 0.73 \\
$k=15$ & 0.1 & 1.68 & 0.10 & 0.90 \\
$k=16$ & $0.1 \cdots$ & $0.90 \cdots$ & $0.10 \cdots$ & 0.90 \\
\hline$k=17$ & 1.51 & 0.10 & 0.73 \\
\hline
\end{tabular}

Abaixo temos a Tabela 6.4 com o resumo däs estināativas. 
Table 6.4 - Resumos das Estimativas

\begin{tabular}{|c|c|c|c|c|c|c|}
\hline & Parâmetro & Média & S.P. & 95\% Intervato de Confia & nça & $|G W|$ \\
\hline \multirow{17}{*}{ Pop1- } & $p_{11}$ & 0.537 & 0.042 & $(0.451 ; 0.61)$ & - & 0.035 \\
\hline & $p_{12}$ & 0.371 & $\theta .036 \cdots$ & $(0.298 ; 0.432)$ & & 0.035 \\
\hline & $\mathrm{p}_{13}$ & 0.862 & 0.025 & $(0.814 ; 0.909)$ & & 0.0306 \\
\hline & $\mathrm{p}_{14}$ & 0.580 & $0.040^{-}$ & $(0.503 ; 0.664)$ & - & 0.0568 \\
\hline & $p_{15}$ & 0.101 & 0.024 & $(0.059 ; 0.153)$ & $\ldots$ & 0.0519 \\
\hline & $\mathrm{p}_{16}$ & 0.068 & 0.021 & $(0.032 ; 0.113)$ & - & 0.0115 \\
\hline & $p_{17}$ & 0.376 & 0.036 & $(0.306 ; 0.446)$ & - & 0.0732 \\
\hline & $p_{18}$ & 0.036 & 0.013 & $(0.014 ; 0.066)$ & & 0.0582 \\
\hline & $p_{19}$ & 0.302 & 0.035 & $(\theta: 233 ; 0.369)$ & $\cdots$ & 0.0189 \\
\hline & $p_{11 a}$ & 0.317 & 0.034 & $(0.263 ; 0.390)$ & & 0.0019 \\
\hline & $p_{111}$ & $0.141^{-}$ & 0.028 & $(0.090 ; 0.206)$ & - & 0.0116 \\
\hline & $p_{112-}$ & 0.681 & 0.036 & $(0.604 ; 0.740)$ & & 0.0426 \\
\hline & $\mathrm{p}_{113}$ & 0.057 & 0.019 & $(0.026 ; 0.098)$ & & 0.0588 \\
\hline & $\mathrm{p}_{114^{-}}$ & 0.068 & 0.020 & $(0.035 ; 0.111)$ & - & 0.0719 \\
\hline & $\mathrm{p}_{115}$ & 0.055 & 0.017 & $(0.027 ; 0.095)$ & & 0.0146 \\
\hline & $p_{116}$ & $\theta: 102$ & $0.023 \cdots$ & $(0.060 ; 0.155)$ & . & 0.0736 \\
\hline & $\mathbf{p}_{117 \ldots}$ & 0.063 & 0.020 & $(0.032 ; 0.112)$ & & 0.0128 \\
\hline \multirow{17}{*}{ Pop2 } & $\mathrm{p}_{21}$ & 0.774 & 0.071 & $(0.568 ; 0.849)$ & - & 0.026 \\
\hline & $\mathrm{p}_{22--}$ & 0.401 & 0.076 & $(0.255 ; 0.552)$ & - & 0.013 \\
\hline & $\mathbf{p}_{23}$ & 0.918 & 0.044 & $(0.816 ; 0.983)$ & - & 0.008 \\
\hline & $\mathbf{p}_{24}-$ & 0.343 & 0.072 & $(0.220 ; 0.497)$ & $\ldots$ & 0.0586 \\
\hline & $\mathrm{p}_{25}$ & 0.095 & 0.043 & $(0.029 ; 0.189)$ & & 0.0719 \\
\hline & $\mathrm{p}_{26}$ & 0.200 & 0.059 & $(0.100 ; 0.330)$ & - & 0.0116 \\
\hline & $\mathrm{p}_{2 z_{-}}$ & 0.598 & 0.080 & $(0.444 ; 0.753)$ & & 0.0736 \\
\hline & $\mathrm{p}_{28}$ & 0.173 & 0.060 & $(0.0719 ; 0.296)$ & $-\cdot$ & 0.0524 \\
\hline & $p_{28}$ & 0.122 & 0.0525 & $(0.041 ; 0.240)$ & & 0.0189 \\
\hline & $\mathrm{p}_{210}$ & 0.357 & 0.080 & $(0.223 ; 0.521)$ & $\cdots$ & 0.0019 \\
\hline & $\mathrm{p}_{211-}$ & 0.173 & 0.059 & $(0.078 ; 0.295)$ & & 0.0121 \\
\hline & $\mathrm{p}_{212}$ & 0.951 & 0.031 & $(0.882 ; 0.993)$ & - & 0.0436 \\
\hline & $\mathbf{p}_{213-}$ & 0.100 & 0.0471 & $(0.025 ; 0.205)$ & - & 0.0388 \\
\hline & $p_{214}$ & 0.121 & 0.051 & $(0.044 ; 0.235)$ & & 0.0519 \\
\hline & $\mathrm{p}_{215}$ & 0.103 & 0.046 & $(0.035 ; 0.209)$ & & 0.013 \\
\hline & $\mathrm{p}_{216}$ & 0.098 & 0.043 & $(0.0301 ; 0.216)$ & & 0.0236 \\
\hline & $\mathrm{p}_{217}$ & 0.128 . & $\theta .0493-$ & $(\theta .0507 ; \theta .229)$ & & 0.0588 \\
\hline
\end{tabular}

Nas Figuras abaixo, temos os gráficos de convergência e as desindades marginais a posterior para os parâmetros. 

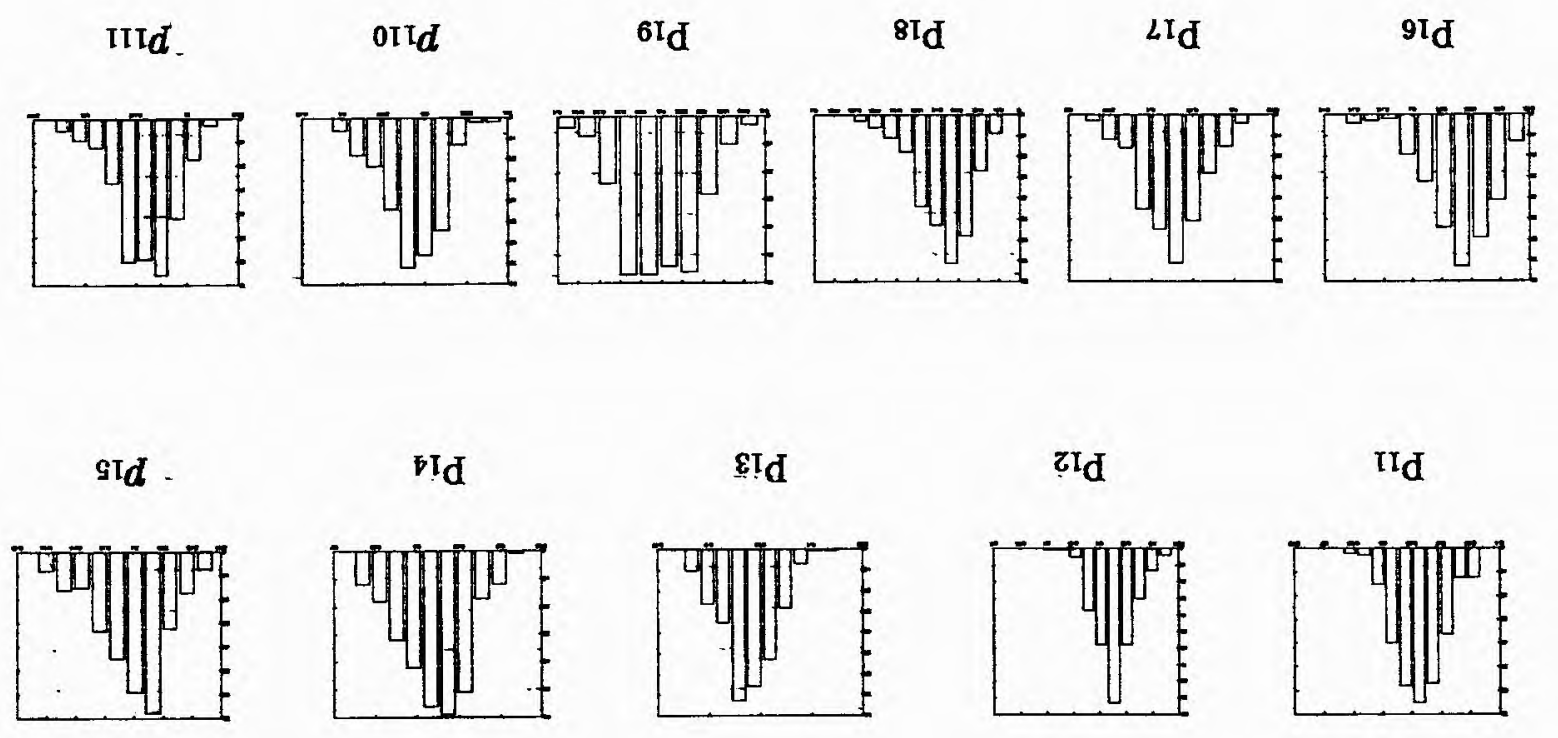

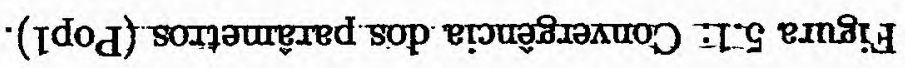

$2 \pi d$

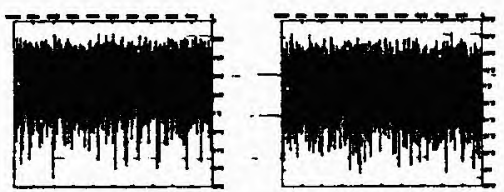

SIId

IIId

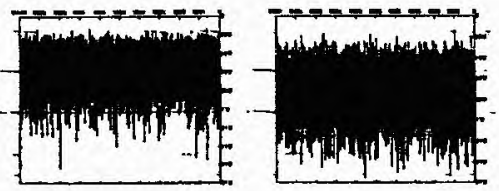

${ }^{6 T} \mathrm{~d}$

${ }^{8 I_{d}}$

${ }^{L} I_{d}$
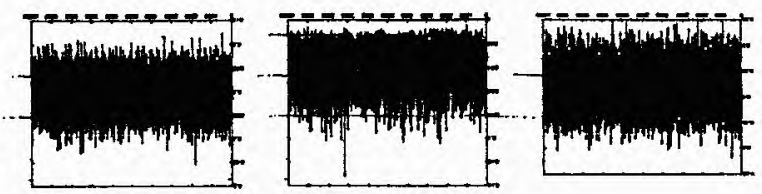

$\boldsymbol{z I}_{\mathbf{d}}$

IId

${ }^{8 I d}$
${ }^{b} \mathrm{I}$

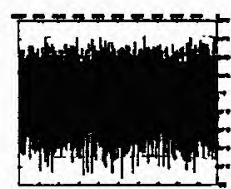

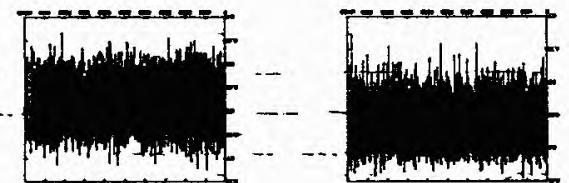
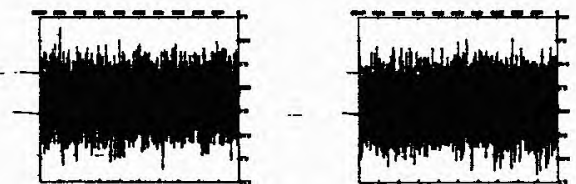


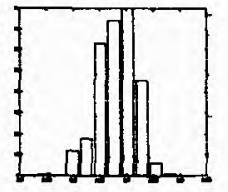

$\mathrm{p}_{\mathrm{i12}}$

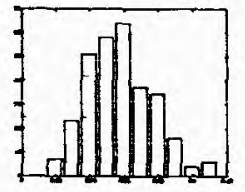

$\mathrm{p}_{113}$

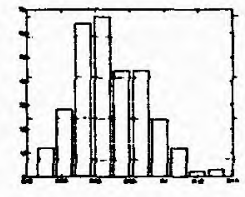

$\mathrm{p}_{1 \mathrm{i} 4}$

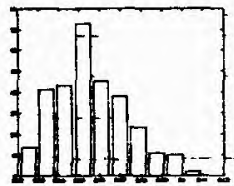

$\mathrm{p}_{\mathrm{i15}}$

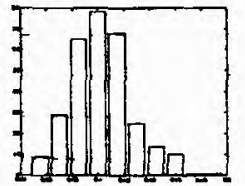

$p_{116}$

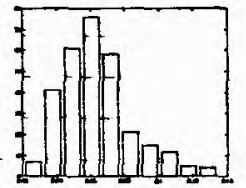

$p_{117}$

Figura 5.2: Densidades Marginais a Posteriori (Pop1).

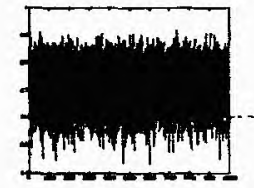

$\mathrm{p}_{\text {ii }}$

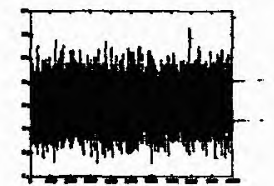

$\mathrm{p}_{12}$

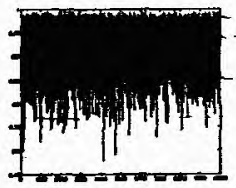

$\mathbf{p}_{13}$

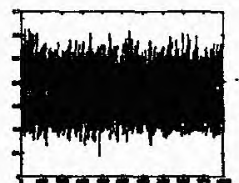

$\mathbf{P}_{14}$

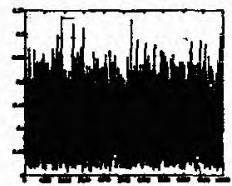

$-p_{15}$

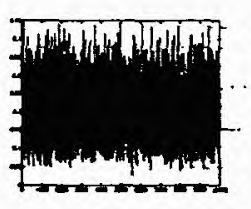

$p_{16}$

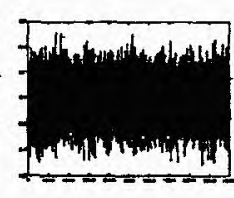

$p_{17}$

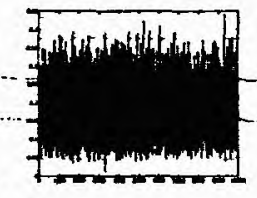

$\mathrm{p}_{18}$

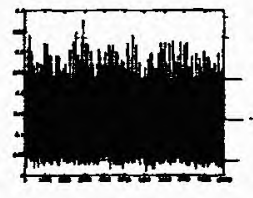

$\mathrm{p}_{19}$

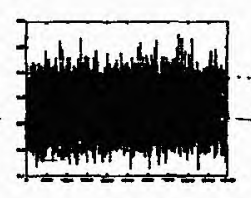

$p_{110}$

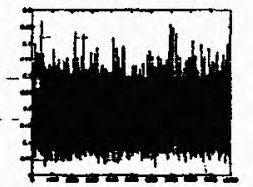

$p_{111}$

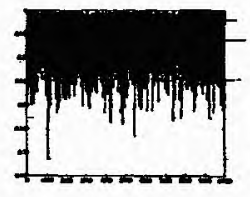

$p_{112}$

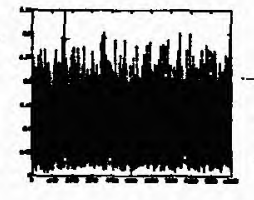

$p_{113}$

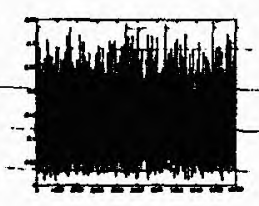

Prt4

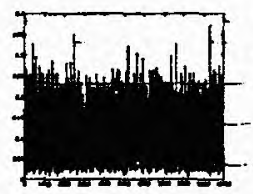

$P_{155}$

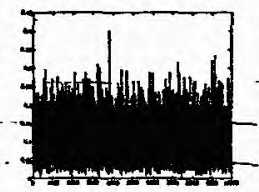

$p_{116}$

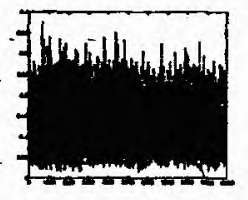

$p_{117}$

Figura 5.3: Convergência dos parâmetros.(Pop2).

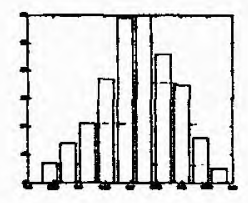

$\mathrm{p}_{11}$

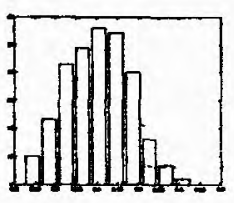

$\mathrm{p}_{12}$

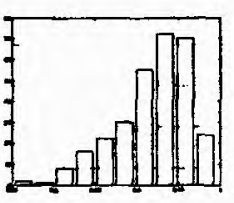

$\mathrm{p}_{\mathrm{i3}}$

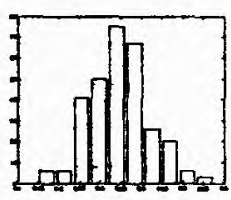

$\mathrm{p}_{14}$

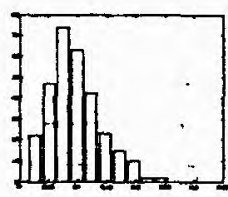

$p_{15}$ 

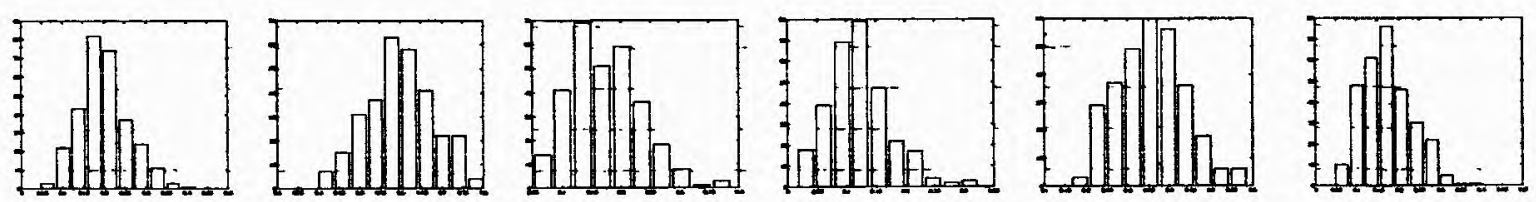

$\mathrm{p}_{16}$

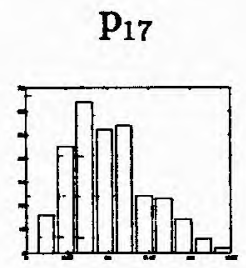

$\mathrm{p}_{112}$ $\mathrm{p}_{18}$

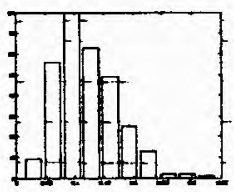

PII4 $p_{19}$

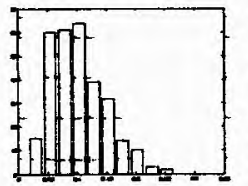

PII5 $^{-}$ $p_{110}$

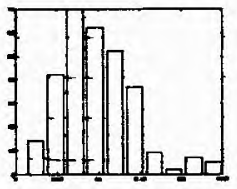

$p_{\mathrm{I} 16}$ $p_{111}$

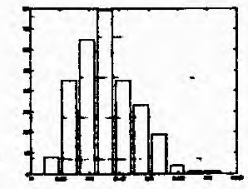

$p_{117}$

Figura 5.4: Densidades Marginais a Posteriori (Pop2).

Abàixo temos a Tabela de classificação,

Tabela 6.5:Tabela de Classificação

Pelà Função Discriminante

\begin{tabular}{c|c|cccc|c}
\hline & & Pö1 & taxa & Pō̄2 & taxa & valor real \\
\hline Membro & Pop1 & 109 & 68.10 & 51 & $31: 80$ & 160 \\
Verdadeiro & Pop2 & 11 & 27.50 & 29 & 72.50 & 40 \\
\hline
\end{tabular}

Assim a taxa de erro aparente $A P E R$ é igual a:

$$
A P E R=\frac{n_{1 M}+n_{2 M}}{n_{1}+n_{2}}=\frac{51+11}{160+40}=0.31
$$

Os resultados da classificação para este procedimento fơram idênticos ao anterior. $O$ procedimento Bayesiano se mostrou como uma outra alternativa para a classificação com tais dados. Acreditamos que com uma boa informação a priori por partè do pesquisador, possamos ter um melhor desempenho. 


\section{Capítulo 6}

\section{Classificação Considerando Dadōs Binários Correlacionados}

No capítulo 5, quando classificamos um objeto ou pessoa a um determinado grupo com base em medidas binárias, não estávamos levando em consideração a correlação entre essas medidas, o que pode ser um erro, pois para um mesmo indivíduo são tomadas várias medidas.

Nesste capítulo, tentamos incorporar o fator correlação entre as medidas para um mesmo indivídūo, inspirados no modelo propostô por Prentice(1988).

Utilizamos uma análise Bayesiana Hierárquica, considerando um efeito aleatório comum a cada indivíduo, que captura a correlação entre as medidas. Num primeiro çaso assumimos uma distribuição normal para tal efeito e em outro caso assumimos uma mistura de distribuições normais.

\subsection{Desenvolvimento dö-Mödelo}

Novamente consideramos o procedimento de classificação para duas popułações. Dentro das características do modelo, considèranda uma amostra $x=\left(x_{1}, \ldots, x_{n_{j}}\right)$ para.çada população $j=1,2$, onde $x_{i}=\left(x_{1 i}, \ldots, x_{k i}, \ldots, x_{q i}\right)$. As funções de verossimilhança ppara cada população são dadas por: 


$$
\begin{aligned}
& L\left(\mathrm{p}_{1}\right)=L\left(\bar{p}_{11}, \ldots, p_{1 q}\right)=\mathrm{H}_{i=1}^{n_{+}} \mathrm{H}_{k=1}^{q} p_{1 k}^{x_{k i}}\left(\mathrm{~L}-p_{1 k}\right)^{1-x_{k i}} \\
& L\left(\mathrm{p}_{2}\right)=L\left(\bar{p}_{21}, \ldots, p_{2 q}\right)=\mathrm{H}_{i=1}^{n_{2}} \Pi_{k=1}^{q} p_{2 k}^{x_{k i}}\left(1-p_{2 k}\right)^{1-x_{k i}},
\end{aligned}
$$

$\operatorname{com} . i=1, \ldots, n_{j}, j=1,2, k=1, \ldots, q$, sendo $n_{1}$ a número de observações da p.ppulação 1 e $n_{2}$ o número de observações dã população 2:

Associando o modelo logístico com efeitos aleatórios, tentamos incorporar a correlação entre as variáveis medidas de um mesmo indivíduo: Portanto para a população 1 tepos,

$$
p_{1 k i}=\frac{e^{\alpha_{1 i}+\beta_{1 k}}}{1+\mathrm{e}^{\alpha_{\Pi i}+\beta_{1 k}}}
$$

$\operatorname{com} i=1, \ldots, n_{1} ; k=1, \ldots, q$.

Aqui, $\alpha_{1 i}$ é visto como efeito aleatório comum a cada indivíduo que captura a correlação entre as medidas. $O$ parâmetro $\beta_{1 k}$, contribui no peso individual da probabilidade de sucesso de cada medida binária:

Da mesma forma para população 2 ,

$$
p_{2 k i}=\frac{\mathrm{e}^{\alpha_{2 i}+\beta_{2 k}}}{1+\mathrm{e}^{\alpha_{2 i}+\beta_{2 k}}}
$$

$\operatorname{com} i=1, \ldots, n_{2} ; k=1, \ldots, q$.

\section{2- Análise Bayesiana-Hierárquica-AssumindōEfeitos Aleatórios Normais}

Aqui, assumimos que $\alpha_{j i}, i=1, \ldots, n_{j}, j=1,2 ;$; são efeitos aleatórios independentes com distribuição normal. Asssim, para cada população temos,

$$
\begin{aligned}
\alpha_{1 i} & \sim N\left(0 ; \sigma_{1}^{2}\right) \\
\alpha_{2 i} & \sim N\left(0, \sigma_{2}^{2}\right),
\end{aligned}
$$

onde $\sigma_{j}^{2}$ é desconhecido com uma distribuição conhecida, portanto $\sigma_{j}^{2}$ é um hiperparâmetro e $N\left(0, \sigma_{j}^{2}\right)$ é uma distribuição a priòri hierárquicā para $\alpha_{j i}, \hat{\imath}=1, \ldots, n_{j} j=1,2$. Sendo assim necessário a atribuição de uma distribuição a priori para estes hiperparâmetros.

Consideramos as seguintes distribuiç̧ōes a priori para $\alpha_{j}^{2}$ e $\beta_{j k}$ em cada população. 
Para a população 1 , vamos ter,

$$
\begin{aligned}
& \sigma_{1}^{2} \sim I G\left(a_{1}, b_{1}\right) ; a_{1}, b_{1} \text { conhecidos. } \\
& \beta_{1 k} \sim N\left(c_{1 k}, d_{1 k}^{2}\right) ; c_{1 k+} d_{1 k} \text {.conhecidos }
\end{aligned}
$$

com $k=1, \ldots, q$, onde $I G$ é uma distrição Gama Invertida com média $\frac{b}{a-1}$ para $a>1 \mathrm{e}$ variância $\frac{b^{2}}{(a-1)^{2}(a-2)}, a>2$.

Assumindo independência a priori para os parâmetros, a distribuição a priori conjunta é da forma,

$$
\pi\left(\alpha_{1}, \sigma_{1}^{2}, \beta_{1}\right) \propto\left(\sigma_{1}^{2}\right)^{-\left(a_{1}+1\right)} \exp \left\{-\frac{b_{1}}{\sigma_{1}^{2}}\right\}\left\{\prod_{i=1}^{n_{1}} \frac{1}{\sqrt{\sigma_{1}^{2}}} \exp \left\{-\frac{1}{2 \sigma_{1}^{2}} \alpha_{1 i}^{2}\right\}\right\}\left\{\prod _ { k = 1 } ^ { q - } \operatorname { e x p } \left\{\frac{1}{2 d_{1 k}^{2}}\left(\beta_{1 k}-c_{1 k}\right)\right.\right.
$$

isto é,

$$
\pi\left(\alpha_{1}, \sigma_{1}^{2}, \beta_{1}\right) \propto\left(\sigma_{1}^{2}\right)^{-\left(a_{1}+\frac{n_{1}}{2}+1\right)} \exp \left\{-\frac{1}{\sigma_{1}^{2}}\left[b_{1}+\frac{1}{2} \sum_{i=1}^{n_{1}} \alpha_{1 ;}^{2}\right]\right\}\left\{\left[\prod_{k=1}^{q} \exp \left\{-\frac{1}{2 d_{1 k}^{2}}\left(\beta_{1 k}-c_{1 k}\right)^{2}\right\}\right\}\right.
$$

onde $\alpha_{1}=\left(\alpha_{11}, \ldots, \alpha_{1 n_{1}}\right), \beta_{i}=\left(\beta_{11}, \ldots ., \beta_{1 q}\right)$.

Assim a distribuição a posteriori conjunta a partir de $6.1,6.2,6.3,6.4 ; 6.5$ e-6.6 é dada por:

$$
\begin{aligned}
\pi\left(\alpha_{1}, \sigma_{1}^{2}, \beta_{1} f D_{1}\right) & \propto \pi\left(\alpha_{1}, \sigma_{1}^{2}, \beta_{1}\right) L\left(\mathrm{p}_{1}\right) \\
& \propto\left(\sigma_{1}^{2}\right)^{-\left(a_{1}+\frac{n_{1}}{2}+1\right)} \exp \left\{-\frac{1}{\sigma_{1}^{2}}\left[b_{1}+\frac{1}{2} \sum_{i=1}^{n_{k}} \alpha_{1 i}^{2}\right]\right\}\left\{\prod_{k=1}^{q} \exp \left\{-\frac{1}{2 \widetilde{d}_{1 k}^{2}}\left(\beta_{1 k}-c_{1 k}\right)^{2}\right\}\right\} \\
& \exp \left\{\sum_{i=1}^{n_{1}} \sum_{k=1}^{q} x_{k i} \alpha_{1 i}+\sum_{i=1}^{n_{k}} \sum_{k=1}^{q} x_{k i} \beta_{1 k}-\sum_{i=1}^{n_{1}} \sum_{k=1}^{q} \ln \left(1+\exp \left\{\alpha_{1 i}+\beta_{1 k}\right\}\right)\right\}
\end{aligned}
$$

onde $D_{1}=\left\{x_{1}, \ldots, x_{n_{1}}\right\}$.

As distribuições condicionais necessárias para o algoritmo Gibbs Sampling são dạdas abaixo.

Para $\alpha_{1 i}$ temos,

$$
\pi\left(\alpha_{1 i} \mid \alpha_{1}^{(i)}, \sigma_{1}^{2}, \beta_{1}, D_{1}\right) \propto \exp \left\{-\frac{1}{2 \sigma_{1}^{2}} \alpha_{1 i}^{2}\right\} \exp \left\{\sum_{k=1}^{q} x_{k i} \alpha_{1 i}-\sum_{k=1}^{q-} \ln \left(1+\exp \left\{\dot{\alpha} \alpha_{1 i}+\beta_{1 k}\right\}\right)^{\prime}\right\}
$$


isto é,

$$
\pi\left(\alpha_{1 i} \mid \alpha_{1}^{(i)}, \sigma_{1}^{2}, \beta_{1}, D_{1}\right) \propto \exp \left\{-\frac{1}{2 \dot{\sigma}_{1}^{2}}\left(\alpha_{1 i}-y_{i} \sigma_{1}^{2}\right)^{2}\right\} \exp \left\{-\sum_{k=1}^{q} \ln \left(1+\exp \left\{\alpha_{1 i}+\beta_{1 k}\right\}\right)\right\}
$$

ou seja,

$$
\pi\left(\alpha_{1 i} \mid \alpha_{1}^{(i)}, \sigma_{1}^{2}, \beta_{1}, D_{1}\right) \propto A\left(y_{i} \sigma_{1}^{2}, \sigma_{1}^{2}\right) \Psi_{\mathrm{I}}\left(\alpha_{1}, \beta_{1}\right),
$$

onde $\Psi_{1}\left(\alpha_{1 i}, \beta_{1}\right)=\exp \left\{-\sum_{k=1}^{q} \ln \left(1+\exp \left\{\alpha_{1 i}+\beta_{1 k}\right\}\right)\right\}, y_{i}=\sum_{k=1}^{q} x_{i k} ; k=1, \ldots, q ; i=$ $1, \ldots, n_{1}$ e $\alpha_{1}^{(i)}=\left(\alpha_{11}, \ldots, \alpha_{1(i-1)}, \alpha_{1(i+1)}, \ldots, \alpha_{1 n_{1}}\right)$.

$\operatorname{Para} \sigma_{1}^{2}$

$$
\pi\left(\sigma_{1}^{2} \mid \boldsymbol{\alpha}_{1}, \beta_{1}, D_{1}\right) \sim I G\left(a_{1}+\frac{n_{1}}{b_{2}}, b_{1}+\frac{1}{2} \sum_{i=1}^{n_{1}} \alpha_{1 i}^{2}\right)
$$

Agora para $\beta_{1 k}$ temos,

$\pi\left(\beta_{1 k} \mid \beta_{1}^{(k)}, \sigma_{1}^{2}, \alpha_{1}, D_{1}\right) \propto\left\{\prod_{k=1}^{q-} \exp \left\{=\frac{1}{2 d_{1 k}^{2}}\left(\beta_{1 k}-c_{1 k}\right)^{2}\right\}\right\} \exp \left\{\beta_{1 k} \sum_{i=1}^{n_{1}} x_{k i}-\sum_{i=1}^{n_{1}} \ln \left(1+\exp \left\{\alpha_{1 k}+\beta_{1 k}\right\}\right)\right\}$, isto é,

$\pi\left(\beta_{1 k} \mid \beta_{1}^{(k)}, \sigma_{1}^{2}, \alpha_{1}, D_{1}\right) \propto \exp \left\{-\frac{1}{2 d_{1 k}^{2}}\left[\beta_{1 k}-\left(c_{1 k}+d_{1 k}^{2} V_{k}\right)\right]^{2}\right\} \exp \left\{-\sum_{i=1}^{n_{1}} \ln \left(1+\exp \left\{\alpha_{1 i}+\beta_{1 k}\right\}\right)\right\}$ ou seja,

$$
\pi\left(\beta_{1 k} \mid \beta_{1}^{(k)}, \sigma_{1}^{2}, \alpha_{1}, B_{1}\right) \propto N\left(c_{1 k^{-}}+d_{1 k}^{2} V_{k}, d_{1 k}^{2}\right) \Psi_{2}\left(\alpha_{1}, \beta_{1 k}\right),
$$

onde $\Psi_{2}\left(\alpha_{1_{2}} \beta_{1 k}\right)=\exp \left\{-\sum_{i=1}^{n_{1}} \ln \left(1+\exp \left\{\alpha_{1 i}+\beta_{1 k}\right\}\right)\right\}, V_{k}=\sum_{i=1}^{n_{1}} x_{k i}$, para $k=1, \ldots, q$ e $\boldsymbol{\beta}_{1}^{(k)}=\left(\beta_{11}, \ldots, \beta_{\mathrm{I}(k-1)}, \beta_{1(k+1)}, \ldots, \beta_{1 q}\right)$.

Dà mesma forma procedemos para a populäção 2:

Dados as formas das distribuições condicionais, vamos utilizar o algoritmo Gibbs Sampling para gerar amostras de $\sigma_{j}^{2}$ e a algoritmo Metropolis-Hasting para gerar amostras de $\alpha_{j i}$ e $\beta_{j k}, j=1,2, i=1, \ldots, n_{j}, k=1, \ldots, ; q$. 
Assim gerado $S$ amostras a partir de Gibbs-Metropolis, encontramos as médias a posteriori dos parâmetros para cada grupo, utiitizando $\sigma$ estimador de Monte Cạrlo.

Portanto, para a população 1 temos,

$$
\hat{\alpha}_{11}, \ldots, \hat{\alpha}_{\mathrm{In}_{1}}, \hat{\sigma}_{1}^{2-}, \hat{\beta}_{11}, \ldots, \hat{\beta}_{1 q}
$$

para a população 2 ,

$$
\hat{\alpha}_{21} ; \ldots, \hat{\alpha}_{2 \pi_{2}}, \hat{\sigma}_{2}^{2}, \hat{\beta}_{21}, \ldots, \hat{\beta}_{2 q}
$$

A partir daí, calculamos

$$
\begin{aligned}
& \hat{\alpha}_{1}=\frac{1}{n_{1}} \sum_{i=1}^{n_{1}-1} \hat{\alpha}_{1 i} \\
& \hat{\alpha}_{2}=\frac{1-}{n_{2}} \sum_{i=1}^{n_{2}} \hat{\alpha}_{2 i}
\end{aligned}
$$

Pơrtanto, temos as seguintes estimativas para cadã população,

$$
\begin{aligned}
& \hat{\alpha}_{1}, \hat{\sigma}_{1}^{2}, \hat{\beta}_{11}, \ldots, \hat{\beta}_{1 q}, \text { para a populaçãa } 1 . \\
& \hat{\alpha}_{2}, \hat{\sigma}_{2}^{2}, \hat{\beta}_{21}, \ldots, \hat{\beta}_{2 q}, \text { para a população } 2 .
\end{aligned}
$$

Logo, vamos ter

$$
\hat{p}_{1 k}=\frac{e^{\hat{\alpha_{1}}+\hat{\beta}_{1 k}}}{1+e^{\hat{\alpha}_{1}+\hat{\beta}_{1 k}}}
$$

e

$$
\hat{p}_{2 k}=\frac{e^{\hat{\hat{\alpha}_{2}}+\hat{\hat{\beta}}_{2 k}}}{1+e^{\hat{\alpha}_{2}+\hat{\beta}_{2 k}}}
$$

Assim,

$$
\begin{aligned}
& \hat{f}_{1}(\mathrm{x})=\prod_{k=1}^{q} \hat{p}_{1 k}^{x_{k}}\left(1-\hat{p}_{1 k}\right)^{\mathrm{T}=x_{k}} \\
& \hat{f}_{2}(\mathrm{x})=\prod_{k=1}^{q \cdots} \hat{p}_{2 k}^{x_{k}}\left(1-\hat{p}_{2 k}\right)^{\mathrm{T}=x_{k}}
\end{aligned}
$$

Novamente a alocação para uma nova observação $\mathrm{x}=\left(x_{1}, \ldots, x_{q}\right)$ será dado por, 
i- Alocar x à população 1 se,

$$
\frac{\hat{f}_{1}(\mathbf{x})}{\hat{f}_{2}(\mathbf{x})}>1
$$

ii- Alocar x à população 2, caso contrário.

\subsection{Modelos de Efeitos Aleatórios com una Mistura de-Distribuições-Normais}

Nesta seção, consideramos os $\alpha_{j i}, j=1,2 ;, i=1, \ldots, n_{j}$. efeitos aleatớrios independentes como uma mistura dē distribuições normais, pois quando consideramos tais efẹitos independentes, podemos ter uma multimodalidade e portanto modelar estes parâmetros com uma única distribuição normat pode não ser muito realístico.

Tentando construir um modelo mais robusto; assmmimos para $\alpha_{j i}$ uma mistura de duas distribuições normais.

Dèsenvolvendo o modelo para a população $I$ temos,

$$
\pi\left(\alpha_{1 i}\right)=\sum_{l=1}^{2-} \theta_{11} \phi_{i}\left(\alpha_{1 i} \mid \mu_{11,}, \sigma_{11}^{2}\right)
$$

ou seja,

$$
\pi\left(\alpha_{1 i}\right)=\theta_{1 \mathrm{it}} \phi_{1}\left(\alpha_{1 i} \mid \mu_{\mathrm{H1}}, \sigma_{\mathrm{1H}}^{2}\right)+\left(1-\theta_{11}\right) \phi_{2}\left(\alpha_{1 i} \mid \mu_{12}, \sigma_{12}^{2}\right) ; i=1, \ldots, n_{j,} \ldots
$$

onde $\phi_{l}$ denata uma densidade normal $N\left(\mu_{1 l}, \sigma_{11}^{2}\right)$, com a restrição $\theta_{11}+\theta_{12}=1$ e $l=1,2$ são índices dos componentes da mistura.

Assumindo as seguintes distribuições a priori para os parâmetros,

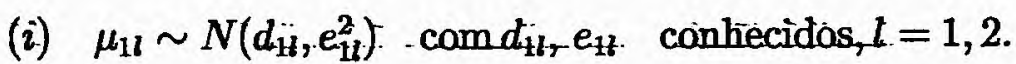

(ìi). $\sigma_{1 l}^{2} \sim I G\left(a_{1 l}, b_{1 b}\right) \quad \operatorname{com} a_{1 l}, b_{1 z}$ conhecidos, $l=1,2$.

(iii) $\beta_{1 k} \sim N\left(c_{1 k}, d_{1 k}^{2}\right) . \quad \operatorname{com} c_{1 k}, d_{1 k}$ conheciđos, $k=1, \ldots, q$.

(iv) $\theta_{11} \sim \operatorname{Beta}\left(f_{1}, g_{1}\right) \quad \operatorname{com} f_{1}, g_{1}$ conhecidos. 
Assumindo independência a priori entre os parâmetros, a distribuição a posteriori conjunta para $\alpha_{1}=\left(\alpha_{11}, \ldots, \alpha_{1 n_{1}}\right), \mu_{1}=\left(\mu_{11}, \mu_{12}\right) ; \theta_{11}, \beta_{1}^{-}=\left(\beta_{11}^{-}, \ldots, \beta_{1 q}\right)$ e $\sigma_{1}=\left(\sigma_{11}^{2}, \sigma_{12}^{2}\right)$ ế dada por,

$$
\begin{aligned}
\pi\left(\alpha_{1}, \beta_{1}, \mu_{1}, \sigma_{1}, \theta_{1 l} \mid D_{1}\right) & \propto \prod_{i=1}^{n_{1}}\left\{\sum_{l=1}^{2} \theta_{1 l} \phi_{l}\left(\alpha_{1 i} \mid \mu_{1 l}, \sigma_{1 l}^{2}\right)\right\} \prod_{l=1}^{2}\left\{\left(\sigma_{1 l}^{2}\right)^{-\left(a_{1 l}+1\right)} \exp \left[-\frac{b_{1 l}}{\sigma_{1 l}^{2}}\right]\right\} \\
& \prod_{l=1}^{2}\left\{\exp \left\{-\frac{1}{2 e_{1 l}}\left(\mu_{1 l}-d_{1 l}^{2}\right)\right\}\right\} \theta_{11}^{f_{i}-1}\left(1-\theta_{11}\right)^{g_{1}-1}\left\{\prod_{k=1}^{q} \exp \left\{-\frac{1}{2 d_{1 k}^{2}}\left(\beta_{1 k}-c_{1 k}\right)^{2}\right\}\right. \\
& \exp \left\{\sum_{i=1}^{n_{1-}} \sum_{k=1}^{q} x_{k i} \alpha_{1 i}+\sum_{i=1}^{n_{1}} \sum_{k=1}^{q} x_{k i} \beta_{1 k}-\sum_{i=1}^{n_{1}} \sum_{k=1}^{q} \ln \left(1+\exp \left\{\alpha_{1 i}+\beta_{1 k}\right\}\right)\right\}
\end{aligned}
$$

onde $D_{1}=\left(\mathbf{x}_{1}, \ldots, \mathbf{x}_{n_{j}}\right)$.

Novamente percebemos que a mistura de distribūições norrnais, dificulta o cálculo das distribuições a posteriori marginais para os parâmetros quando consideramos uma análise Bayesiana. Assim, para simplificar as distribuições condicionais necessárias para o algoritmo Gibbs Sampling consideramos, como no capítulo 4, a incorporação de variáveis lätentes (ver Tanner. \&. Wòng, 1987) dadas por $z_{i}=\left(z_{i 1}, z_{i 2}\right) ; z_{i 1}+z_{i 2}=1, i=1, \ldots, n_{j}$ onde $z_{i l}$ tem uma distribuição Binomial $b\left(1, h_{i i}\right)$ onde,

$$
h_{i l}=\frac{\theta_{i l} \phi_{l}\left(\alpha_{1 i} \mid \mu_{1 l}, \sigma_{1 l}^{2}\right)}{\sum_{l=1}^{2} \theta_{1 l} \phi_{l}\left(\alpha_{1 i} \mid \mu_{1 l}, \sigma_{1 l}^{2}\right)}
$$

Assim, considerando uma amostra $z=\left(z_{1}, \ldots, z_{n_{1}}\right)$, tepos

$$
\begin{aligned}
\pi(\mathrm{z}) & =\prod_{i=1}^{n_{1}}-h_{i 1}^{z_{i 1}}\left(1-h_{i 1}\right)^{z_{i 2}} \\
& =\frac{\prod_{i=1}^{n_{1}} \prod_{l=1}^{2 \cdot-}\left[\theta_{1 l} \phi_{l}\left(\alpha_{1 i} \mid \mu_{1 l}, \sigma_{1 l}^{2}\right)\right]^{z_{i k}}}{\prod_{i=1}^{n_{1}-}\left\{\sum_{l=1}^{2} \theta_{1 l} \phi_{i}\left(\alpha_{1 i} \mid \mu_{1 l}, \sigma_{1 i}^{2}\right)\right\}}
\end{aligned}
$$

onde

$$
z_{i 1}= \begin{cases}1 & \text { com probabilidade } . \\ 0 . & \text { com probabilidade. } 1-h_{i 1} .\end{cases}
$$

Combinanda (6.16) com (6.18) temos.a seguinte distribuição a posteriori conjunta, 
$\pi\left(\alpha_{1}, \beta_{1}, \mu_{1,}, \sigma_{12}, \theta_{11} \mid z, D_{1}\right) \propto \prod_{i=1}^{\pi \mathrm{T}} \prod_{l=1}^{2}\left[\theta_{11} \phi_{l l}\left(\alpha_{1 i} \mid \mu_{1 !}, \sigma_{1 !}^{2}\right)\right]^{z_{i l}} \prod_{l=1}^{2}\left\{\left(\sigma_{1 l}^{2}\right)^{-\left(a_{1 l}+1\right)} \exp \left[-\frac{b_{1 !}}{\sigma_{1 l}^{2}}\right]\right\}$

$$
\begin{aligned}
& \prod_{l=1}^{2}\left\{-\exp \left\{-\frac{1}{2 e_{1 l}}\left(\mu_{1 L}-d_{1 l}^{2}\right)\right\} \theta_{11}^{f_{i}-1}\left(1-\theta_{11}\right)^{g_{1}-1}\left\{\prod_{k=1}^{q} \exp \left\{-\frac{1}{2 d_{1 k}^{2}}\left(\beta_{1 k}-c_{1 k}\right)^{2}\right\}\right.\right. \\
& \exp \left\{\sum_{i=1}^{n_{1}-q} \sum_{k=1}^{q} x_{k i} \alpha_{1 i}+\sum_{i=1}^{n_{1}} \sum_{k=1}^{q} x_{k i} \beta_{1 k}-\sum_{i=1}^{n_{1}} \sum_{k=1}^{q} \ln \left(1+\exp \left\{\alpha_{1 i}+\beta_{1 k}\right\}\right)\right\}
\end{aligned}
$$

De (6.19), encontramos as distribivições condicionais necessária para @algoritmo Gijbbs

Sampling.

Para $\alpha_{1 i}$ teinos,

$$
\begin{gathered}
\pi\left(\alpha_{1 i} \mid \alpha_{1}^{(i)}, \beta_{1}, \mu_{1}, \sigma_{1}, \theta_{11}, \mathrm{z}, D_{1}\right) \propto \exp \left\{-\frac{z_{i 1}}{2 \sigma_{11}^{2}}\left(\alpha_{1 i}-\mu_{11}\right)^{2}\right\} \exp \left\{-\frac{z_{i 2}}{2 \sigma_{12}^{2}}\left(\alpha_{1 i}-\mu_{12}\right)^{2}\right\} \\
\exp \left\{\sum_{k=1}^{q} x_{k i} \alpha_{1 i}-\sum_{k=1}^{q} \ln \left(1+\exp \left\{\alpha_{1 i}+\beta_{1 k}\right\}\right)\right\}
\end{gathered}
$$

isto é,

$$
\begin{aligned}
\pi\left(\alpha_{1 i} \mid \alpha_{1}^{(i)}, \beta_{12}, \mu_{1}, \sigma_{1}, \theta_{11}, \mathrm{z}, D_{1}\right) & \propto \exp \left\{-\frac{1}{2}\left(\frac{\sigma_{12}^{2} z_{i 1}+\sigma_{11}^{2} z_{i 2}}{\sigma_{11}^{2} \sigma_{12}^{2}}\right)\left(\alpha_{i 1}-\frac{z_{i 1} \mu_{11} \sigma_{12}^{2}+z_{i 2} \mu_{12} \sigma_{12}^{2}}{\sigma_{12}^{2} z_{i 1}+\sigma_{11}^{2} z_{i 2}}\right)\right\} \\
& \exp \left\{-\sum_{k=1}^{q} \bar{x}_{k i} \alpha_{1 i}-\sum_{k=1}^{q} \ln \left(1+\exp \left\{\alpha_{1 i}+\beta_{1 k}\right\}\right)\right\},
\end{aligned}
$$

ou seja,

$$
\begin{aligned}
& \pi\left(\alpha_{1 i} \mid \alpha_{1}^{(i)} \beta_{1,} \mu_{12}, \sigma_{1}, \theta_{11}, z, D_{1}\right) \propto N\left(\frac{-z_{i 1} \mu_{11} \sigma_{12}^{2}+z_{i 2} \mu_{12} \sigma_{12}^{2}}{\sigma_{12}^{2} z_{i 1}+\sigma_{11}^{2} z_{i 2}}, \frac{\sigma_{11}^{2} \sigma_{12}^{2}}{\sigma_{12}^{2} z_{i 1}+\sigma_{11}^{2} z_{i 2}}\right) \Psi_{3}\left(\alpha_{1 i,} \bar{\beta}_{1}\right), \\
& \text { onde } \\
& \Psi_{3}\left(\alpha_{1 i}, \beta_{1}\right)=\exp \left\{\sum_{k=1}^{q} x_{k i} \alpha_{1 i}-\sum_{k=1}^{q} \ln \left(1+\exp \left\{\alpha_{1 i}+\beta_{1 k}\right\}\right)\right\} \\
& \text { para } i=1, \ldots, n_{1} \text { e } \alpha_{1}^{(i)}=\left(\alpha_{11}, \ldots, \alpha_{T(i-1)}, \alpha_{1(i+1)}, \ldots, \alpha_{i n_{1}}\right) .
\end{aligned}
$$

Para $\sigma_{1 l}^{2}$ temos,

$$
\pi\left(\sigma_{1 l}^{2} \mid \alpha_{1}, \beta_{1}, \mu_{1}, \theta_{11}, \mathrm{z}, D_{1}\right) \propto\left(\sigma_{1 l}^{2}\right)-\left(a_{11}+\frac{\sum_{i=1}^{n_{1} z_{i l}}+1}{2}+1\right) \exp \left\{-\frac{1}{\sigma_{1 l}^{2}}\left(\frac{\sum_{i=1}^{n_{1}} z_{i l}\left(\alpha_{1 i}-\mu_{11}\right)^{2}}{2}+b_{1 l}\right)\right\}
$$


ou seja,

$$
\left(\sigma_{11}^{2} \mid \alpha_{1}, \beta_{1}, \mu_{1}, \theta_{11}, z, D_{1}\right) \sim I G\left(a_{11}+\frac{\sum_{i=1}^{n_{1}} z_{i t}}{2}+1, \frac{\sum_{i=1}^{n_{1}} z_{i t}\left(\alpha_{1 i}-\mu_{11}\right)^{2}}{2}+b_{1 l}\right)
$$

$l=1,2$.

Para $\theta_{11}$ temos,

$$
\left(\theta_{11} \mid \boldsymbol{\alpha}_{1}, \boldsymbol{\beta}_{1}, \boldsymbol{k}_{12}, \boldsymbol{\sigma}_{12} z_{2} D_{1}\right) \sim \operatorname{Beta}\left(f_{1}+\sum_{i=1-}^{n_{1}} z_{i 1}, g_{1}+\sum_{i=1}^{n_{1}} z_{i 2}\right)
$$

Para $\beta_{1 k}$ temos,

$\pi\left(\beta_{1 k} \mid \beta_{1}^{(k)}, \sigma_{1}, \alpha_{1}, \mu_{1}, \theta_{11}, \mathrm{z}, D_{1}\right) \propto \exp \left\{-\frac{1 \cdot}{2 d_{1 k}^{2-}}\left[\beta_{1 k}-\left(c_{1 k}+d_{1 k}^{2} V_{k}\right)\right]^{2}\right\} \exp \left\{-\sum_{i=1}^{n_{1}} \ln \left(1+\exp \left\{\alpha_{1 i}+\beta_{1 k}\right\}\right.\right.$ ou seja,

$$
\pi\left(\beta_{1 k} \mid \beta_{1}^{(k)}, \sigma_{1}, \boldsymbol{\alpha}_{1} ; \boldsymbol{\mu}_{1}, \theta_{\mathrm{II}}, \mathbf{z} ; D_{1}\right) \propto N\left(c_{\mathrm{IK}}+d_{1 k}^{2} V_{k}\right) \Psi_{4}\left(\alpha_{1} ; \beta_{\mathrm{I} k}\right)
$$

onde,

$\Psi_{4}\left(\alpha_{1}, \beta_{1 k}\right)=\exp \left\{-\sum_{i=1}^{n_{1}} \ln \left(1+\exp \left\{\alpha_{1 i}+\beta_{1 k}\right\}\right)\right\} ; V_{k}=\sum_{i=1}^{n_{1}} x_{k i} ; k=1, \ldots, q \mathbf{e}$ $\beta_{1}^{(k)}=\left(\beta_{11}, \ldots, \beta_{1(k-1)}, \beta_{1(k+1)} ; \ldots, \beta_{1 q}\right)$.

Para $\mu_{1 l}$

$$
\left(\mu_{11} \mid \sigma_{1}, \alpha_{1}, \beta_{1}, \theta_{11}, \mathrm{z}, D_{1}\right) \sim N\left(\frac{d_{1 !} \sigma_{1 l}^{2}+e_{1 b}^{2} \sum_{i=I}^{n_{1}} z_{i l} \alpha_{1 i}}{\sigma_{1 l}^{2}+e_{1 l}^{2} \sum_{i=1}^{n_{1}} z_{i l}}, \frac{e_{1 l}^{2} \sigma_{1 l}^{2}}{\sigma_{1 l}^{2}+e_{1 l}^{2} \sum_{i=1}^{n_{1}} z_{i l}}\right)
$$

$l=1,2$.

Da mesma forma procedemos para a população 2 .

Novamente utilizamos Gibbs-Metropolis para gerarmos as amostras dos parâmetros. $\mathrm{O}$ algoritmo é dado por:

i- Atualizar emcada iteração a vetor $z_{2}=\left(z_{i}, \ldots, z_{n_{g}}\right)$, a partir da distribuị̧̧̃o binomial $b\left(1, h_{1 j}^{\bar{j}}\right)$.

ii- Usar o algoritmo Gibbs-Metropolis para gerar amostras de $\boldsymbol{\alpha}_{1}, \boldsymbol{\beta}_{1}, \boldsymbol{\mu}_{1}$ e $\sigma_{1}$ a partir das distribuições condicionais a posteriori. 
ii- repetir os passos i e ii de acordo com o número de iterações.

Gërado $S$ amostras, utilizando o estimador de Moute Carło, teremos para cada pppulação,

$$
\hat{\alpha}_{11}, \ldots, \hat{\alpha}_{1 n_{1}}, \hat{\sigma}_{1}^{2}, \hat{\beta}_{1 \Gamma}, \ldots, \hat{\beta}_{1 q}, \text { parz-a-pepulação } 1
$$

e

$$
\hat{\alpha}_{21}, \ldots, \hat{\alpha}_{2 n_{2}}, \hat{\sigma}_{2}^{2}, \hat{\beta}_{21}, \ldots, \hat{\beta}_{2 q} ; \text { para-a população } 2
$$

Novamente, calculamos

$$
\begin{aligned}
& \hat{\alpha}_{1}^{-}=\frac{1}{n_{1}} \sum_{i=\mathrm{I}}^{n_{1}} \hat{\alpha}_{1 i} \\
& \hat{\alpha}_{2}=\frac{1}{\bar{n}_{2}} \sum_{i=\mathrm{I}}^{n_{2}} \hat{\alpha}_{2 i}
\end{aligned}
$$

Portanto,

$$
\hat{\alpha}_{1}, \hat{\sigma}_{1}^{2}, \hat{\beta}_{\mathrm{TI}}, \ldots, \hat{\beta}_{1 q}, \text { para-a pepulação } 1 .
$$

$\hat{\alpha}_{2}, \hat{\sigma}_{2}^{z}, \hat{\beta}_{2 \uparrow}, \ldots, \hat{\beta}_{2 q}$, para-a-pepulaçãa 2 .

Logo,

$$
\hat{p}_{1 k}=\frac{e^{\widehat{\alpha_{1}}+\hat{\beta}_{1 k}}}{1+e^{\alpha_{1}+\hat{\beta}_{1 k}}}
$$

e

$$
\hat{p}_{2 k}=\frac{e^{\hat{\alpha}_{\overline{2}}+\hat{\beta}_{2 k}}}{1+e^{\hat{\alpha}_{2}+\hat{\beta}_{2 k}}}
$$

Assim,

$$
\begin{aligned}
& \hat{f}_{1}(\mathrm{x})=\prod_{k=1}^{q} \hat{p}_{1 k}^{x_{k}}\left(1-\hat{p}_{1 k}\right)^{\mathrm{r}-x_{k}} \\
& \hat{f}_{2}(\mathrm{x})=\prod_{k=1}^{q} \hat{p}_{2 k}^{x_{k}}\left(1-\hat{p}_{2 k}\right)^{\mathrm{I}=x_{k}}
\end{aligned}
$$

Da mesma forma anterior, a alocação de uma nova abservação $\mathrm{x}=\left(x_{1}, \ldots, x_{q}\right)$ fica: 
i- Alöcar x à população I se,

$$
\frac{\hat{f}_{1}(\mathrm{x})}{\hat{f}_{2}(\mathrm{x})}>1
$$

ii- Alocar $\mathrm{x}$ à população 2, caso contrário.

\subsection{Exemplo Ilustrativo}

Vamos considerar novamente o problemra proposto no capítulo 5.

Para o primeiro modelo, efeito aleatório visto como uma distribuição normal, o procedimento é dēscrito abaixo.

Pära o exemplo, temos döis conjuntos de dados para as respectivas populações. Para a população 1 , indivíduos que sobrevivem, temos $n_{1}=160$ observações, ou seja, vamos ter $\alpha_{1,1}, \ldots, \alpha_{1 ; 160}, \beta_{1,1}, \ldots, \beta_{1,17}$ e $\alpha_{11}^{2}$, teremos ao todo 178 parâmetros. Para a população 2, indivíduos que não sobrevivem, temos $n_{2}=40$ observações, ou seja, vamos ter $\alpha_{2,1}, \ldots, \alpha_{1,40} \beta_{2,1}, \ldots, \beta_{2,17}$ e $\sigma_{21}^{2}$, portanto teremos 58 parâmeţros.

Os procedimentos de simulação são idênticos ao descrito no capítulo 4; aqui também utilizamos o Software Ox.

Os parâmetros a priori para as duas populações são dađōs na Tabela 6.1 abaixo,

Table 6.1 - Parâmetros a Priori

\begin{tabular}{|c|c|cc|}
\hline & & hiperparâmetro 1 & hiperpafâmetro 2 \\
\hline \multirow{6}{*}{ Pop1 } & $\sigma_{1}^{2}$ & $15 \sigma$ & I.92 \\
\cline { 2 - 4 } & $\beta_{11}$ & 0.15 & 0.052 \\
& $\beta_{12}$ & -0.51 & 0.017 \\
& $\beta_{15}$ & 1.83 & 0.14 \\
& $\beta_{14}$ & 0.32 & 0.009 \\
& $\beta_{15}$ & -2.2 & 0.077 \\
& $\beta_{16}$ & -2.6 & 0.091 \\
& $\beta_{17}$ & -0.51 & 0.017 \\
& $\beta_{18}$ & -3.24 & 0.13 \\
& $\beta_{19}$ & -0.84 & 0.029 \\
& $\beta_{110}$ & -0.75 & 0.026 \\
& $\beta_{111}$ & -1.78 & 0.062 \\
& $\beta_{112}$ & 0.76 & 0.026 \\
& $-\beta_{113}$ & -2.82 & 0.13 \\
& $\beta_{114}$ & -2.60 & 0.12 \\
& $\beta_{115}$ & -2.82 & .13 \\
& $\beta_{116}$ & -2.19 & 0.11 \\
& $\beta_{117}$ & -2.7 & 0.13 \\
\hline
\end{tabular}


Table 6.1 - Continuação

\begin{tabular}{|c|c|cc|}
\hline & & hiperparámetro 1 & - biperparämetro 2 \\
\hline & $\sigma_{12}^{2-}$ & $150^{-}$ & 1.92 \\
\hline$\beta_{21}$ & 0.96 & 0.04 \\
& $\beta_{22}$ & -0.40 & 0.02 \\
$\beta_{23}$ & 2.51 & 0.01 \\
$\beta_{24}$ & $-0.62-$ & 0.03 \\
& $\beta_{25}$ & -2.2 & 0.13 \\
& $\beta_{26}$ & -1.38 & 0.08 \\
& $\beta_{27}$ & 0.40 & 0.02 \\
& $\beta_{28}$ & -1.55 & 0.09 \\
& $\beta_{29}$ & -1.94 & 0.11 \\
& $\beta_{210}$ & -0.62 & 0.04 \\
& $\beta_{211}$ & -1.55 & 0.09 \\
& $\beta_{212}$ & 2.94 & 0.14 \\
& $\beta_{213}$ & -2.2 & 0.13 \\
$\beta_{214}$ & -1.94 & 0.11 \\
& $\beta_{215}$ & -2.19 & 0.13 \\
& $\beta_{216}$ & -2.19 & 0.13 \\
$\beta_{\overline{217}}$ & -1.94 & 0.11 \\
\hline
\end{tabular}

Dada a grande quantidade de parâmetros por parte dos efeitos aleatórios e verificando que a estimativa de tais de uma forma geral foram nulos, iremos ilustrar alguns resultados dados que os restantes seguem aproximadamente os mesmos valores.

Tablè 6.2 = Resumos däs Estimnätivas

\begin{tabular}{|c|c|c|c|c|c|c|}
\hline & Parâmetro. & Média. & S.P.- & 95\% Intervalo-de-Confiança & $|G W|$ & Aceitaçă \\
\hline \multirow{6}{*}{ Pop1 } & $\alpha_{11}$ & -0.010 & $0 . I I$ & $(-0.24 ; 0.21)$ & 0.035 & $69 \%$ \\
\hline & $\alpha_{12}$ & -0.02 & 0.11 & $(-0.23 ; 0.18)$ & 0.01 & $70 \%$ \\
\hline & $\vdots$ & $\vdots$ & $\vdots$ & $\vdots$ & $\vdots$ & $\vdots$ \\
\hline & $\alpha_{180}$ & 0.005 & 0.10 & $(-0.22 ; 0.22)$ & 0.008 & $70 \%$ \\
\hline & $\vdots$ & $\vdots$ & $\vdots$ & $\vdots$ & $\vdots$ & $\vdots$ \\
\hline & $\alpha_{160}$ & -0.024 & 0.10 & $(-0.22 ; 0.17)$ & 0.02 & $70 \%$ \\
\hline \multirow{6}{*}{ Pop2 } & $\alpha_{21}$ & 0.046 & 0.10 & $(-0.14 ; 0.24)$ & 0.02 & $65 \%$ \\
\hline & $\alpha_{22}$ & 0.01 & 0.11 & $(-0.19 ; 0.25)$ & 0.06 & $64 \%_{-}$ \\
\hline & $\vdots$ & $\vdots$ & $\vdots$ & $\vdots$ & $\vdots$ & \\
\hline & $\alpha_{220}$ & 0.02 & 0.10 & $(-0.17 ; 0.23)$ & 0.04 & $65 \%$ \\
\hline & $\vdots$ & $\vdots$ & $\vdots$ & $\vdots$ & $\vdots$ & $\vdots$ \\
\hline & $\alpha_{240}$ & -0.027 & 0.11 & $(-0.26 ; 0.18)$ & 0.03 & $64 \%$ \\
\hline
\end{tabular}

E as estimativas,

$$
\begin{aligned}
& \hat{\alpha}_{1}=-0.00018 \\
& \hat{\alpha}_{2}=0.0016
\end{aligned}
$$


Na Tabela 6.3 abaixo, temos a continuação dos resultados para os parâmetros de ambas populaçōes,

Table 6.3 - Resumos das Estimativas

\begin{tabular}{|c|c|c|c|c|c|c|}
\hline & Parámetro - & Média- & S.D. & 95\% Intervalo de Confiança. & |GW & Aceitação \\
\hline \multirow{18}{*}{ Popl ${ }^{--}$} & $\beta_{11}$ & 0.15 & 0.052 & $(0.05 ; 0.25)$ & 0.025 & $74 \%$ \\
\hline & $\beta_{12}$ & -0.50 & 0.12 & $(-0.72 ;-0.23)$ & 0.0106 & $44 \%$ \\
\hline & $\beta_{13}$ & 1.84 & 0.21 & $(1.45 ; 2.26)$ & 0.012 & $38 \%$ \\
\hline & $\beta_{14}$ & 0.32 & 0.09 & $(0.14 ; 0.50)$ & 0.0568 & $45 \%$ \\
\hline & $\beta_{15}$ & $-2.22-$ & $0.26^{-}$ & $(-2.83 ;-1.71)$ & $0.049^{-}$ & $37 \%$ \\
\hline & $\beta_{16}$ & -2.64 & 0.27 & $(-3.21 ;-2.21)$ & 0.0415 & $45 \%$ \\
\hline & $\beta_{17}$ & -0.51 & 0.11 & $(-0.76 ;-0.29)$ & 0.0312 & $45 \%$ \\
\hline & $\beta_{18}$ & -3.3. & 0.4 & $(-4.11 ;-2.60)$ & 0.0512 & $61 \%$ \\
\hline & $\beta_{10}$ & $-0: 85$ & 0.15 & $(-1.14 ;-0.55)$ & 0.0239 & $30 \%$ \\
\hline & $\beta_{110}$ & -0.759 & 0.14 & $(-1.05 ;-0.48)$ & 0.029 & $33 \%$ \\
\hline & $\beta_{111}$ & -1.80 & 0.21 & $(-2.2 ;-1.45)$ & 0.0116 & $30 \%$ \\
\hline & $\beta_{112}$ & a.75 & 0.14 & $(0,495 ; 1.035)$ & 0.0226 & $77 \%$ \\
\hline & $\beta_{113}$ & $-2.85-$ & $0.31 z$ & $(-3.45 ;-2.25)$ & 0.0558 & $52 \%$ \\
\hline & $\beta_{114}$ & -2.67 & 0.29 & $(-3.29 ;-2.14)$ & 0.0419 & $46 \%$ \\
\hline & $\beta_{115}$ & -2.86 & 0.33 & $(-3.55 ;-2.26)$ & 0.0346 & $41 \%$ \\
\hline & $\beta_{116}$ & -2.22 & 0.23 & $(-2.74 ;-1.78)$ & 0.0736 & $35 \%$ \\
\hline & $\beta_{117}$ & -2.74 & 0.30 & $(-3,4 ;-2.21)$ & 0.0428 & $30 \%$ \\
\hline & $\sigma_{1}^{2}$ & 0.012 & 0.0011 & $(0.0108 ; 0.015)$ & 0.00128 & $\because$ \\
\hline \multirow{18}{*}{ Pop2 } & $\beta_{21}$ & 0.98 & 0.23 & $(0.48 ; 1.39)$ & 0.016 & $31 \%$ \\
\hline & $\beta_{22}$ & -0.41 & 0.13 & $(-0.64 ;-0.13)$ & 0.023 & $82 \%$ \\
\hline & $\beta_{23}$. & 2.64. & 0.56 & $(1.54 ; 3.67)$ & 0.018 & $66 \%$ \\
\hline & $\beta_{24}$ & $-0.65^{--}$ & 0.184 & $(-1: 00 ;-0.27)$ & 0.0126 & $71 \%$ \\
\hline & $\beta_{25}$ & -2.25 & 0.45 & $(-3.24 ;-1.43)$ & 0.0319 & $66 \%$ \\
\hline & $\beta_{26}$ & -1.41 & 0.33 & $(-2.08 ;-0.79)$ & 0.0416 & $63 \%$ \\
\hline & $\beta_{27}$ & 0.40 & 0.13. & $(0.15 ; 0.68)$ & 0.0236 & $68 \%$ \\
\hline & $\beta_{28}$ & -1.57 & 0.31 & $(-2.14 ;-0.93)-$ & 0.0424 & $64 \%$ \\
\hline & $\beta_{29}$ & -1.99 & 0.41 & $(-2.76 ;-1.2)$ & 0.0589 & $67 \%^{-}$ \\
\hline & $\beta_{210}$ & -0.62 & 0.17 & $(-0.97 ;-0.24)$ & 0.0101 & $71 \%$ \\
\hline & $\beta_{211}$ & -160 & 0.32 & $(-2.27 ;-0.958)$ & 0.0421 & $69 \%$ \\
\hline & $\beta_{212}$ & $3.04-$ & $0.64-$ & $(1.80 ; 4.30)$ & 0.0436 & $66 \%$ \\
\hline & $\beta_{213}$ & -2.223 & 0.438 & $(-3.094 ;-1.4)$ & 0.0688 & $66 \%$ \\
\hline & $\beta_{214}$ & -1.96 & 0.36 & $(-2.66 ;-1.28)$ & 0.0488 & $66 \%$ \\
\hline & $\beta_{215}$ & -2.25 & 0.39 & $(-2.99 ;-1.50)$ & 0.0719 & $66 \%$ \\
\hline & $\beta_{216}$ & $-2.26-$ & 0.42 & $(-3.12 ;-1.50)$ & 0.043 & $66 \%$ \\
\hline & $\beta_{217}$ & $-1.97^{-}$ & 0.40 & $(-2.80 ;-1.32)$ & 0.0436 & $66 \%$ \\
\hline & $\sigma_{2}^{2}$ & 0.012 & 0.0011 & $(0.0108 ; 0.015)$ & 0.00128 & - \\
\hline
\end{tabular}

Abaixo temos gráficos de convergência e densidades marginais a posteriori para alguns parâmetros das duias populações. 


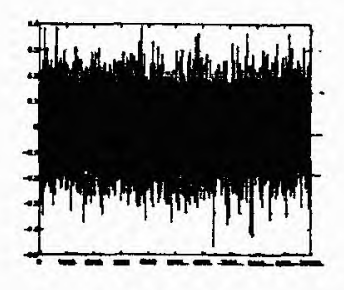

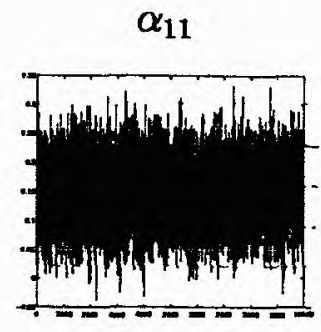

$\beta_{1+}$

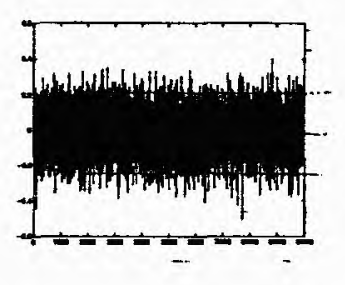

$\alpha_{12}$

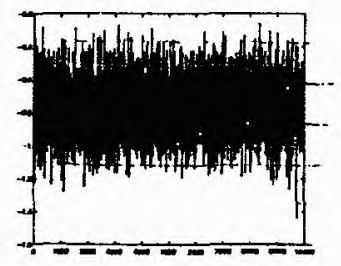

$\beta_{110}$

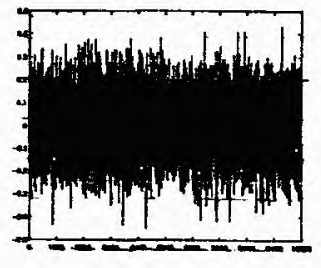

$\alpha_{180}$

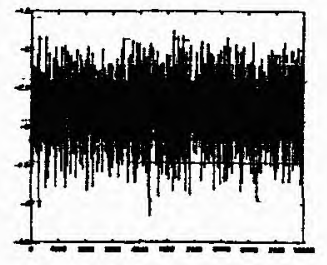

$\beta_{417}$
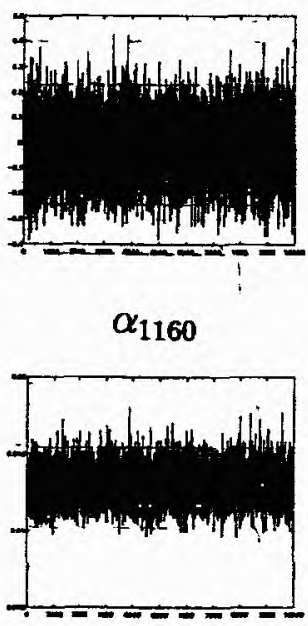

$\sigma_{1}^{2}$

Figura 6.t: Convergência dos parâmetros_(Pop1).

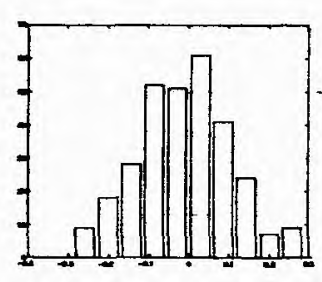

$\alpha_{11}$

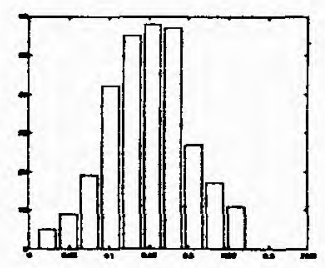

$\beta_{\text {ii }}$

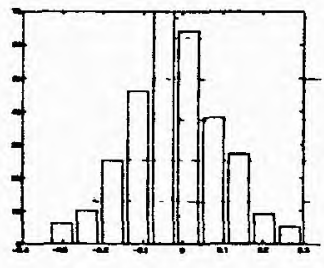

$\alpha_{12}$

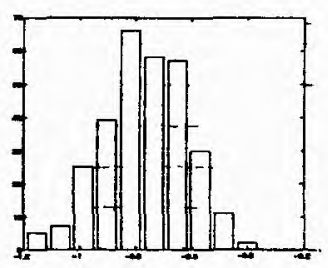

$\beta_{\text {ito }}$

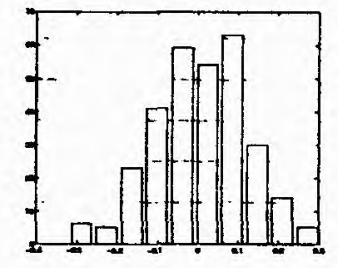

$\alpha_{180}$

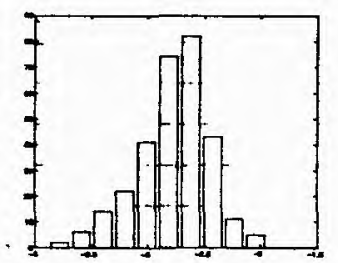

$\beta_{i 17}$

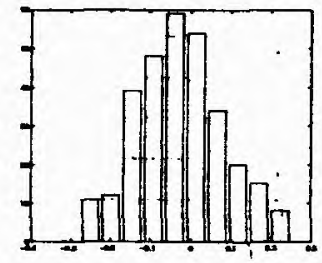

$\alpha_{1160}$

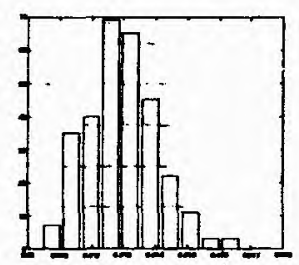

$\sigma_{1}^{2}$

Figura 6.2: Densidades Marginais a. Posteriori (Pop1). 


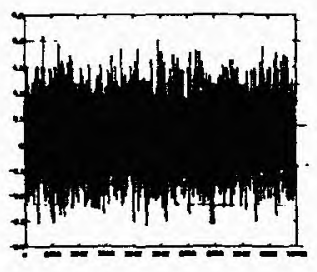

$\alpha_{11}$

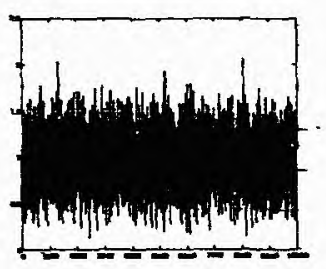

$\beta_{\mathrm{ir}}$

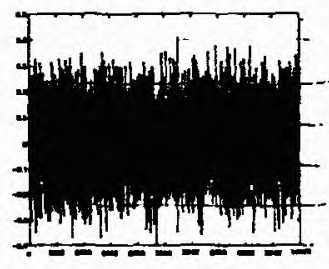

$\alpha_{12}$

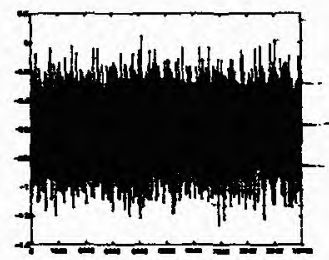

$\beta_{110-}$

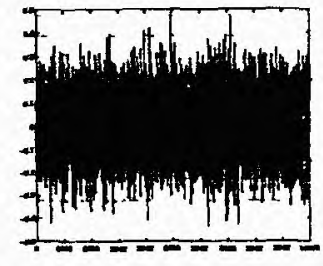

$\alpha_{180}$

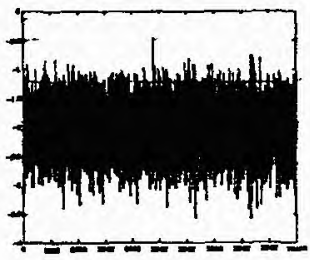

$\beta_{417}$

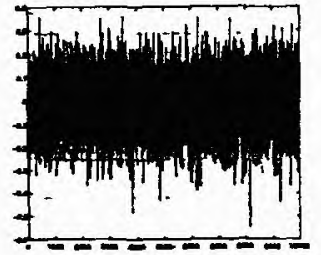

$\alpha_{1160}$

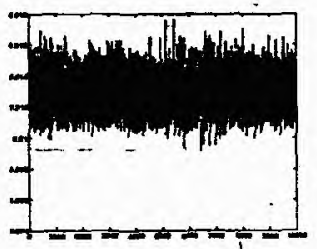

$\sigma_{1}^{2}$

Figura 6.3: Conyergência dos parâmetros.(Pop2).

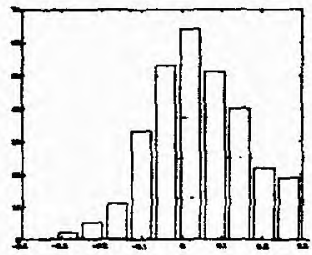

$\alpha_{11}$

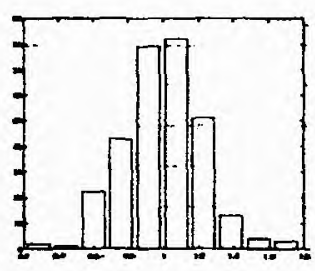

$\beta_{11}$

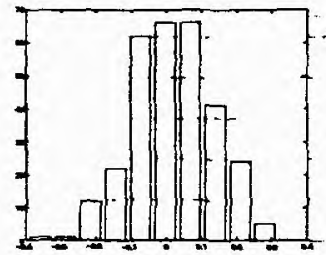

$\alpha_{12}$

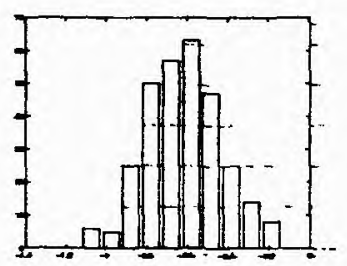

$\beta_{1+1}$

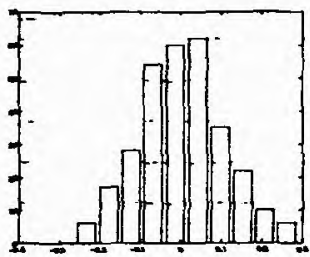

$\alpha_{180}$

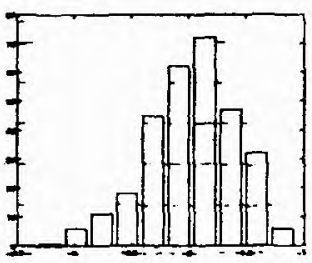

$\beta_{117}$

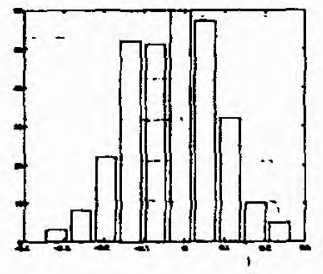

$\alpha_{1160}$

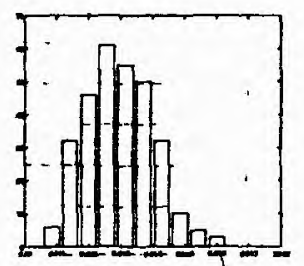

$\sigma_{1}^{2}$

Fìgura 6.4: Dènsidades Mărginais a Posteriori (Pọp2.

Para as duras populações temos bons resultados para o critério-de convergência; percebese também um índice muito elevado na taxa de aceitação. O fato é que para um álto número de parâmetros, fica muito difícil uma análise caso a caso, portanto as alterações nos hiperparâmetros são feitas de forma coletiva, o que pode levar a casos extremos de aceitação ou rejeição no algoritmo:

A Tabela de classificação é dada abaixo, 
Tabela 6.4:Tabela de Classificação

Pela Regra Discriminante

\begin{tabular}{c|c|cccc|c}
\hline & & Popt & taxa & Pop̀ & taxa & valor real \\
\hline Membro & Pop1 & $109^{-}$ & $68.10^{\circ}$ & 51 & 31.80 & 160 \\
Verdadeiro & Pop2 & 11 & 27.50 & $29-$ & 72.50 & 40 \\
\hline
\end{tabular}

Assim a taxa de erro aparente $A P E R$ é igual a:

$$
A P E R=\frac{n_{1 M}+n_{2 M}}{n_{1}+n_{2}}=\frac{51+11}{160+40}=0.31
$$

Os resultados da classificação foram iguais ao procedtimento anterior, onde consideramos independência entre as medidas para um mesmo indivíduo. Isto se deve em função dos efëitos aleatórios nulos estimados pelo modelo.

Para o segundo caso, considerando o efeito aleatório como uma mistura de 2 distribuições normais, vamos ter para a população $1 \alpha_{1 ; 1}, \ldots, \alpha_{1,169}, \beta_{1,1}, \ldots, \beta_{1,17}, \mu_{11}, \mu_{12}, \sigma_{11}^{2}, q_{12}^{2}$ e $\phi_{11}$, ou seja, teremos ao todo 182 parâmetros. Para a população 2 , vamos ter $\alpha_{2,1}, \ldots, \alpha_{1,40}$, $\beta_{2,1}, \ldots, \beta_{2,17}, \mu_{21}, \mu_{22}, \sigma_{21}^{2}, \sigma_{22}^{2}$ e $\phi_{21}$, portanto teremos.62 parâmetros.

Novamente, usamos os procedimentos de simulação descrito no capítuto 4 , com a utilização do Software "Ox".

Os parâmetros a priori para as duaas populações são dados na Tabela 6.5 abạixo,

Table 6.5 - Parâmetros a Priori

\begin{tabular}{|c|c|c|c|}
\hline & & \multicolumn{2}{|c|}{ hiperparâmetro 1 - hiperparâmetro 2} \\
\hline \multirow{18}{*}{ Popl - } & \multirow{2}{*}{$\begin{array}{l}\mu_{11} \\
\mu_{12}\end{array}$} & \multirow{2}{*}{$\begin{array}{l}0.003 \\
0.001\end{array}$} & \multirow{2}{*}{$\begin{array}{l}0.002 \\
0.001\end{array}$} \\
\hline & & & \\
\hline & $\sigma_{11}^{2}$ & 149 & 1.91 \\
\hline & $\sigma_{12}^{2}$ & 150 & 1.92 . \\
\hline & $\phi_{1 i}$ & 15 & 15 \\
\hline & $\beta_{11}$ & 0.15 & 0.052 \\
\hline & $\beta_{12}$ & -0.51 & -0.17 \\
\hline & B1s. & 1.83. & 0.64 . \\
\hline & $-\beta_{\text {IS }}$ & $\theta .32-$ & 0.114 \\
\hline & $\beta_{18}$ & -2.2 & -0.77 \\
\hline & $\beta_{10}$ & -2.6 & -0.91 \\
\hline & $\beta_{12}$ & -0.51 & -0.17 \\
\hline & $\beta_{18}$ & $-3.24 \cdots$ & -1.13 \\
\hline & $\beta_{19}$ & -0.84 & -0.29 \\
\hline & $\beta_{110}$ & -0.75 & -0.26 \\
\hline & $\beta_{11}$ & -1.78 & -0.62 \\
\hline & $\beta_{112}$ & $0.76-$ & 0.26 \\
\hline & $\beta_{113}$ & -2.82 & -0.98 \\
\hline
\end{tabular}


Table 6.5 - Continuação

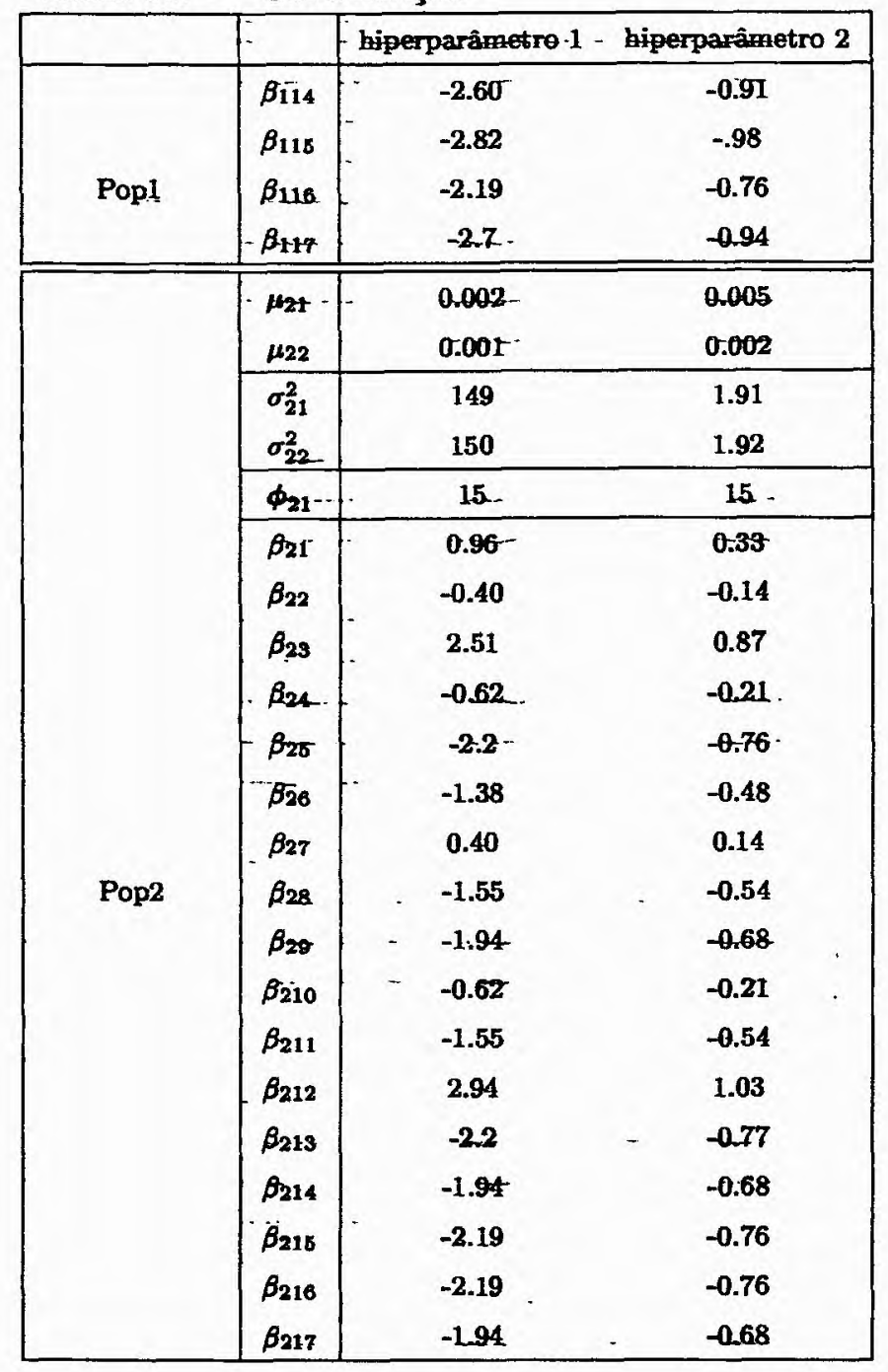

Novamente, dada a grande quantidade de parâmetros; por parte dos efeitos aleatópios, e verificando que a estimativa de tais, de uma forma geral foram nulos, iremos ilustrar alguns resultados.

Table 6.6 - Resumos das Estimativas

\begin{tabular}{|ccccccc|}
\hline & Parâmetro & Média & S.D. & 95\% Intervalo de Confiança & $|G W|$ & Aceitaçä́ \\
\hline & $\alpha_{11}$ & -0.014 & 0.105 & $(-0.21 ; 0.18)$ & 0.025 & $92 \%$ \\
& $\vdots$ & $\vdots$ & $\vdots$ & $\vdots$ & $\vdots$ & $\vdots$ \\
Pop1 & $\alpha_{180}$ & 0.017 & 0.11 & $(-0.17 ; 0.23)$ & 0.01 & $79 \%$ \\
& $\vdots$ & $\vdots$ & $\vdots$ & $\vdots$ & $\vdots$ & $\vdots$ \\
& $\alpha_{1160}$ & -0.019 & 0.108 & $(-0.229 ; 0.183)$ & 0.008 & $75 \%$ \\
\hline & $\alpha_{21}$ & 0.069 & 0.11 & $(-0.14 ; 0.28)$ & 0.02 & $72 \%$ \\
& $\vdots$ & $\vdots$ & $\vdots$ & $\vdots$ & $\vdots$ & $\vdots$ \\
& $\alpha_{220}$ & 0.005 & 0.119 & $(-0.213 ; 0.228)$ & 0.06 & $85 \%$ \\
& $\vdots$ & $\vdots$ & $\vdots$ & $\vdots$ & $\vdots$ & $\vdots$ \\
& $\alpha_{240}$ & -0.038 & 0.118 & $(-0.277 ; 0.197)$ & 0.04 & $83 \%-1$ \\
\hline
\end{tabular}


$\mathrm{E}$ as estimativas, $\hat{\alpha}_{1}=0.00106$ e $\hat{\alpha}_{2}=0.00338$

Table 6.7 - Continuação do Resumo das Estimativas

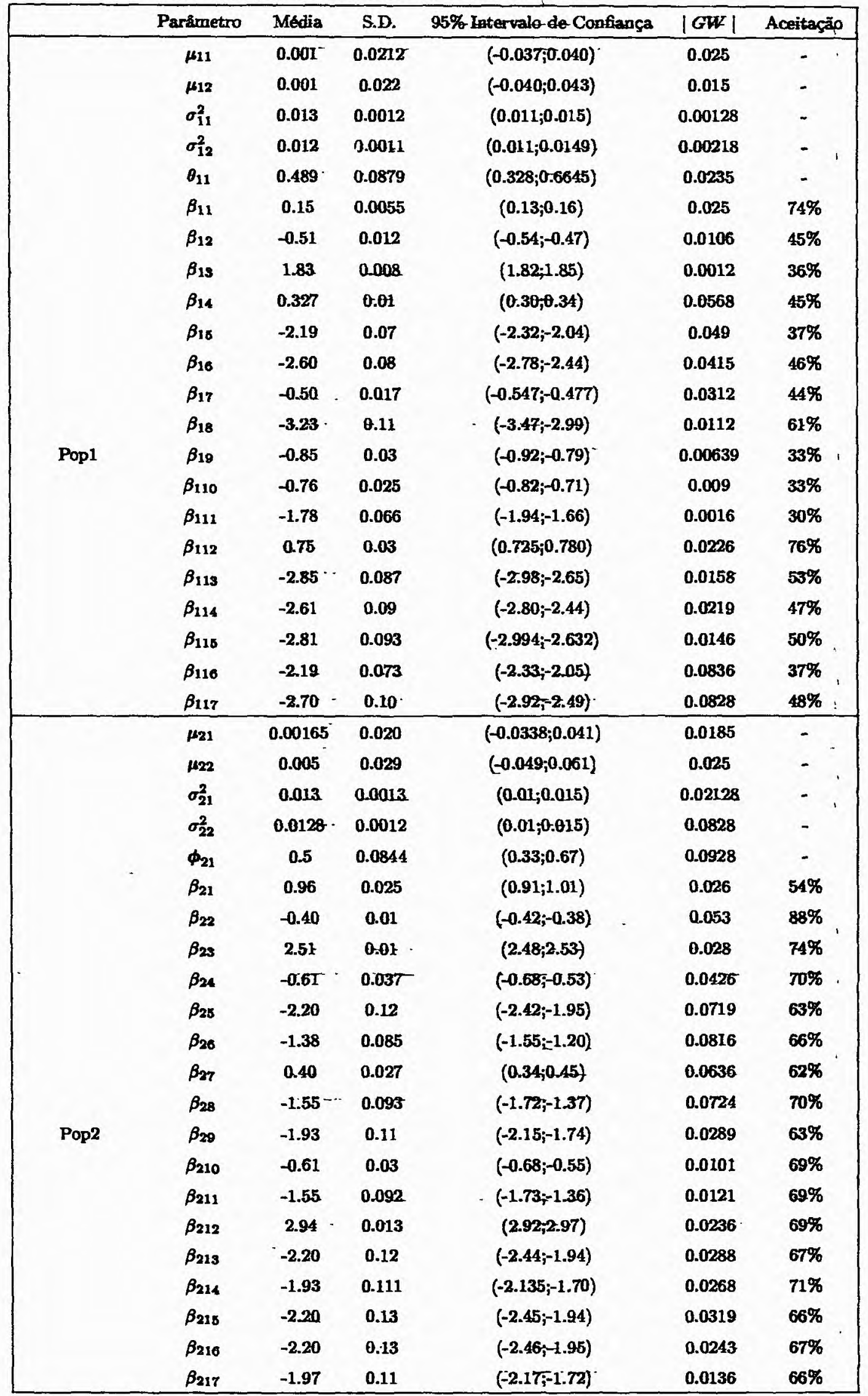


Abaixo temos gráficos de convergência e densidades marginais a posteriori para alguns parâmetros das duas populações.

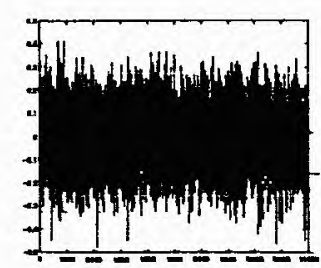

$\alpha_{11}$

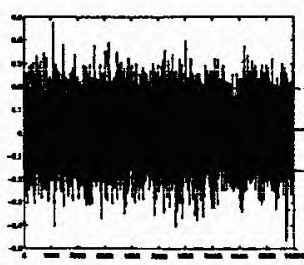

$\alpha_{180}$

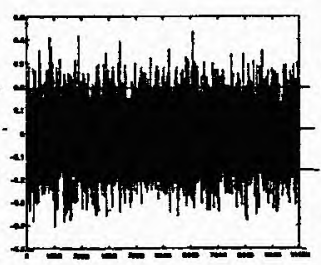

$\alpha_{1160}$

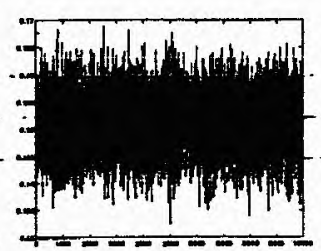

$\beta_{11}$

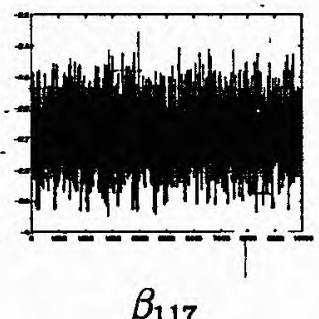

$\beta_{117}$

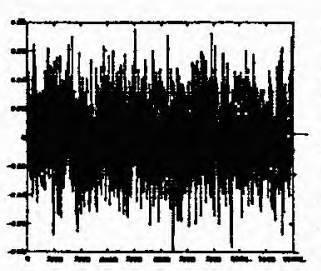

$\mu_{11}$

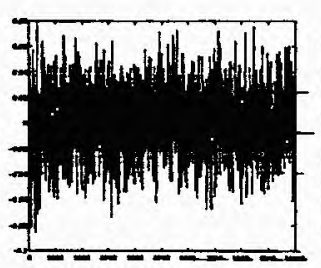

$\mu_{12}$

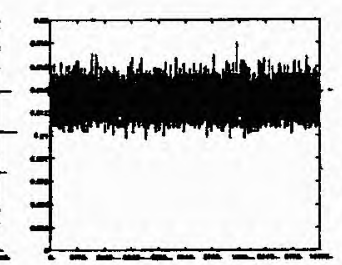

$\sigma_{11}^{2}$

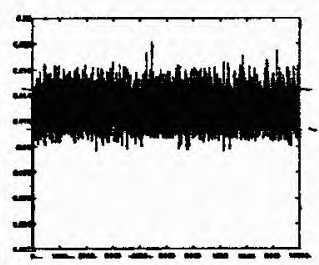

$\sigma_{12}^{2}$

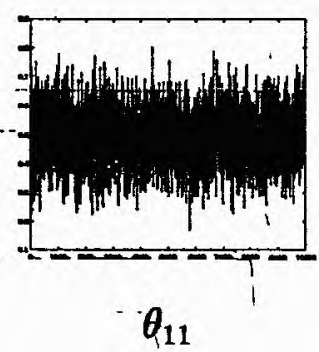

Figura 6.5: Convergência dos parâmetros (Pop1).

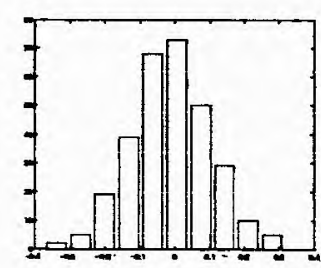

$\alpha_{11}$

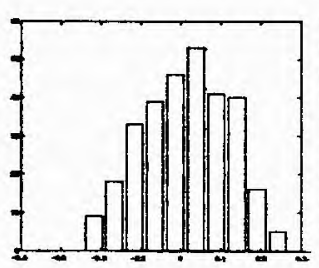

$\alpha_{180}$

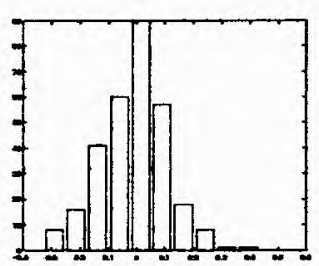

$\alpha_{1160}$

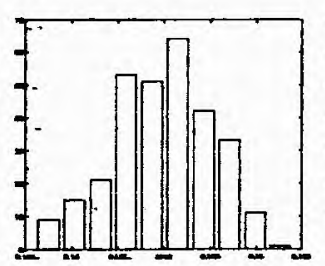

$\beta_{11}$

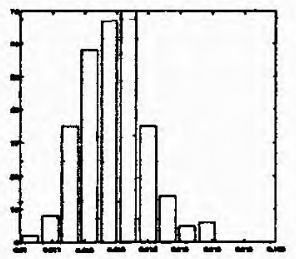

$\sigma_{12}^{2}$

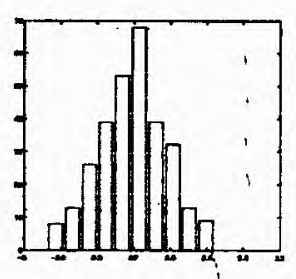

$\beta_{117}$

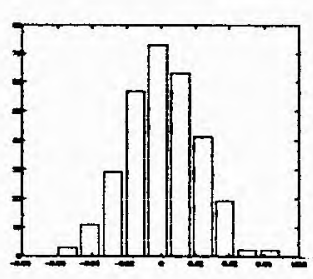

$\mu_{11}$

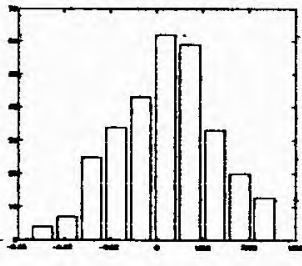

$\mu_{12}$

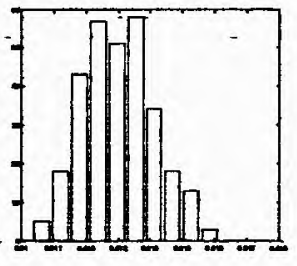

$\sigma_{11}^{2}$

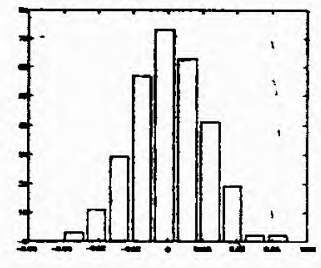

$\theta_{11}$

Figura 6.6: Densidades Marginais a. Posteriori (Pop1). 


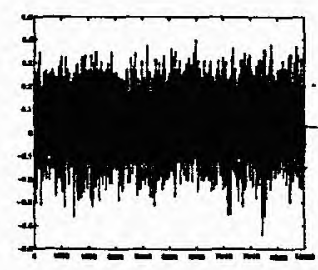

$\alpha_{11}$

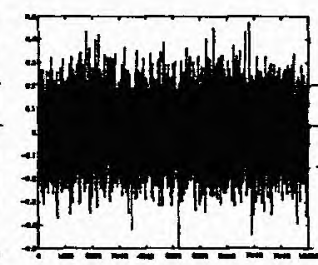

$\alpha_{120}$

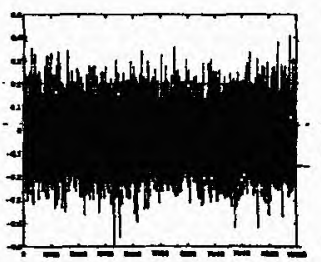

$\alpha_{140}$

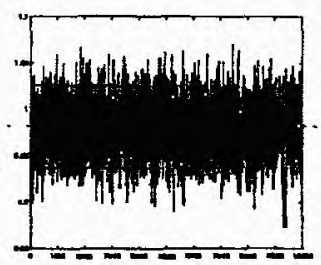

$\beta_{11}$

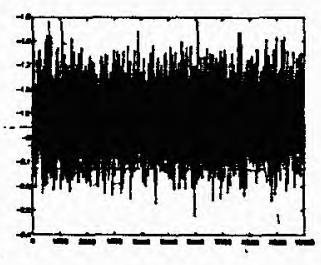

$\beta_{117}$

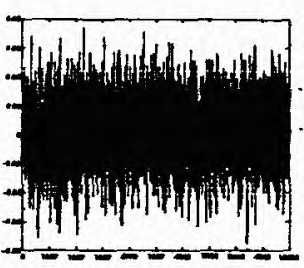

$\mu_{11}$

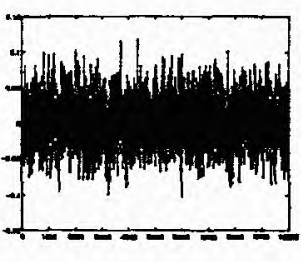

$\mu_{12}$

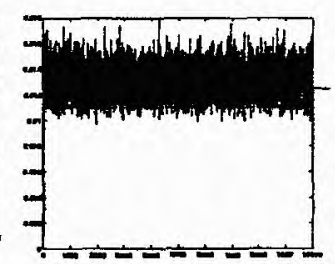

$\sigma_{11}^{2}$

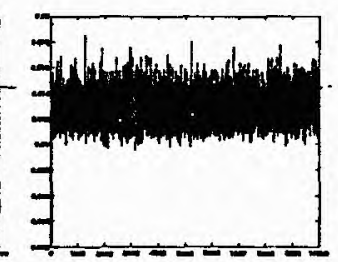

$\sigma_{12}^{2}$

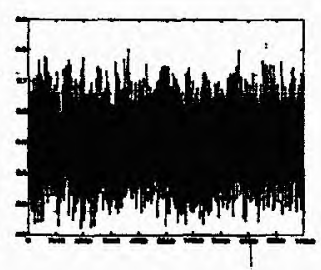

,$\theta_{11}$

Figura 6.7: Convergência dos parâmetros (Pop2).

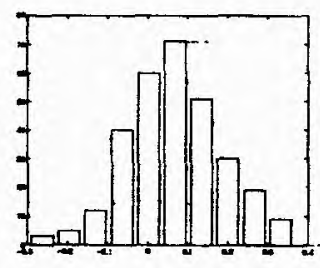

$\alpha_{11}$
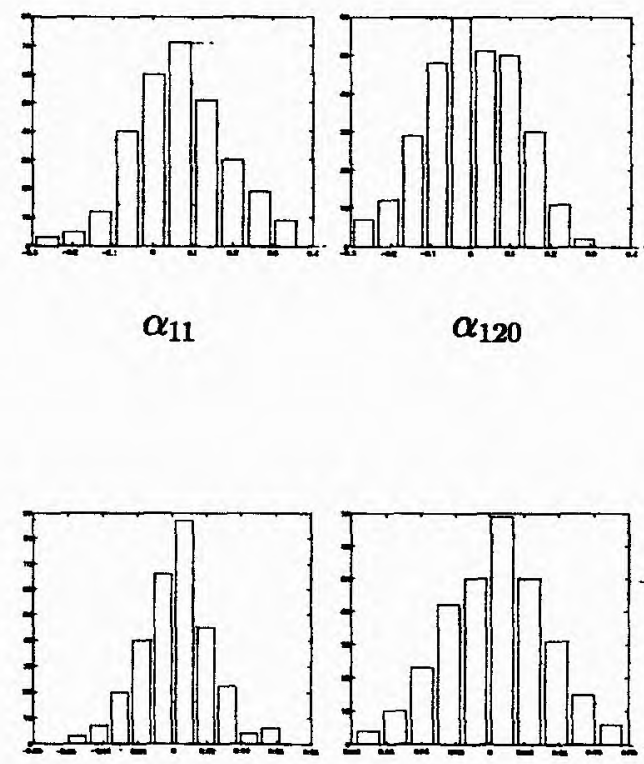

$\mu_{11}$ $\alpha_{120}$

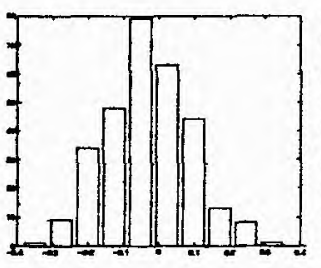

$\alpha_{140}$

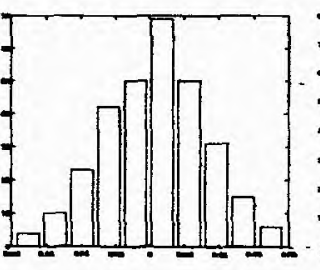

$\mu_{12}$

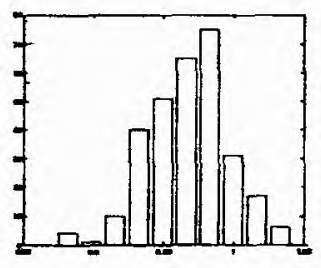

$\beta_{11}$

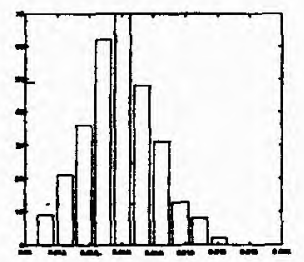

$\sigma_{12}^{2}$

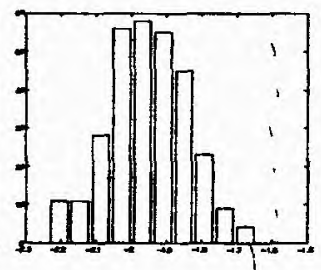

$\beta_{117}$

Figura 6.8: Densidades Marginais a Posteriori (Pop2).

Para as duas populações temos bons resultados para o critério de convergência, percębese também um índice muito elevado na taxa de aceitação. $O$ fato é que para um alto 
número de parâmetros, fica muito difícil uma análise caso a caso, portanto as alterações nos hiperparâmetros são feitas de forma coletiva, o que pode levar a casos extremos de aceitação ou rejeição no algoritmo.

A tabela de classificação é dada abaixo,

Tabela 6.7:Tabela de Classificação

Pela Regra Discriminante

\begin{tabular}{c|c|cccc|c}
\hline & & Pop1 & taxa & Pop2 & taxa & valor real \\
\hline Membro & Pop1 & 109 & 68.10 & 51 & 31.80 & 160 \\
Verdadeiro & Pop2 & 11 & 27.50 & 29 & 72.50 & 40 \\
\hline
\end{tabular}

Assim a taxa de erro aparente $(A P E R)$ é iguạl a:

$$
A P E R=\frac{n_{1 M}+n_{2 M}}{n_{1}+n_{2}}=\frac{51+11}{160+40}=0.31
$$

Os resultados da classificação foram semelhantes ao procedimento anterior, onde consideramos independência entre as medidas para um mesmo indivíduo.

O modelo aqui proposto também não conseguiu detectar a correlação, ou seja, os efeitos aleatórios estimados foram nulos. 


\section{Capítulo 7}

\section{Conclusões Finais e Propostas}

\section{Futuras}

Nesta dissertação consideramos muitos tópicos isolados que devem ser discutidos individualmente.

Cönsiderando o problema de classificação com dados transformados, ficou evidente a grande importância dos métodos de transformação no uso de procedimentos de claśsificação. Esse trabalho também nos forneceu uma experimentação prática onde conseguimos enxergar melhor o comportamenta dos métados tradicionais de classificação(F.D.ł. e F.D.Q.) diante de dados sem transformação e transformados. O procedimento Bayesiano (TBoxl) se mostrou viável dentro de aspectos teóricos e computacionais. Tivemos algumas dificuldades com o procedimento (TBox2), que podem ser abordadas em traballhos futuros onde podemos tentar outras parametrizações para tal transformação.

O uso de misturas de distribuições normais parà o problema de classificação se mostrou uma execente opção, quando os dàdos apresentam características multimodais. Os problemas computacionais referentes a implementação e o tempo de processamento do algoritmo Gibbs Sampling foram completamente viabilizados com a utilização do Software "Ox". No entanto faltou tempo disponível para experimentação com dados reais. Futuramente podemos trabalhar com mistura de distribuições mais gerais como por exemplo a pistribuição $t-d e-S t u d e n t$ Multivariada.

Considerando a classificação com dados binários independentes, verificamos um desempenho equivalente entre o método de classificação geral e a proposta bayesiana apre- 
sentada. A incorporação da informação a priori é de grande importância dentro dessa metodologia, pois temos que conhecer bem as populações previamente para podemos classificar com eficiência novas observações. No exemplo apresentado, nos baseamos exclusivamente na amostra preliminar, talvez seja esse o motivo da equivalência de resultados. Acreditamos que com boas informaçōes a priori adquiridas com o pesquisador possamos melhorar a taxa de classificação.

Quando tentamos incorporar o efeito aleatório em cada indivíduo, objetivando capturar a correlação entre as variáveis binárias do conjunto de dados proposto, não conseguimos melhorar os resultados da classificação. Uma das hipóteses é que este conjunto de dados não possue tais características, ou seja as variáveis para um mesmo indivíduo não são correlacionadas. Também entendemos que pode se tratar de um modelo não muito apropriado para esta finalidade. Considerando o efeito aleatório como uma mistura de normais, também não conseguimos melhorar os resultados. Uma proposta futura neste caso, seria utilizar o modelo de regressão logística para a classificação e incorporar o fator correlação entre as covariáveis, tomanda como base o modeloproposto por Prentice(19898). 


\section{Apêndice A}

\section{ALGORITMO GIBBS SAMPLING}

O algoritmo Gibbs sampling foi introduzido formalmente por Geman e Geman (1984), quando propuseram um esquema de amostragem de- distribuições explorando as distribuições condicionais completas através de um algoritmo iterativo que define uma cadeia de Markov.

$O$ algoritmo Gibbs sampling é essencialmente um esquema iterativo de amostragem de uma cadeia de Markov cujo núcleo de transição é formado pelas distribuições condicionais completas:

Descrição do método:

Suponha que a distribuição de interesse é $\pi(\theta)$ onde $\theta=\left(\theta_{1}, \theta_{2}, \ldots, \theta_{p}\right)$. Considere ainda que as densidades condicionais completas a posteriori $\pi_{i}\left(\theta_{i} \mid \theta_{-i}\right), \quad i=1, \ldots, p$ estão disponíveis sob forma padronizada. Podemos então gerar as amostras como segue:

(i) inicializemos o contador de iterações da cadeia $j=1$ e arbitremos vatóres iniçiais $\theta^{(0)}=\theta_{1}^{(0)}, \theta_{2}^{(0)}, \ldots, \theta_{p}^{(0)}$.

(ii) obtemos um novo valor $\theta^{(j)}=\theta_{1}^{(j)}, \theta_{2}^{(j)}, \ldots, \theta_{p}^{(j)}$ a partir de $\theta^{(j-1)}$ através de-sucessivas gerações de valores

$$
\begin{aligned}
& \theta_{1}^{(j)} \sim \pi\left(\theta_{1} \mid \theta_{2}^{(j-1)}, \ldots, \theta_{p}^{(j-1)}\right) \\
& \theta_{2}^{(j)} \sim \pi\left(\theta_{2} \mid \theta_{1}^{(j)}, \theta_{3}^{(j-1)}, \ldots, \theta_{p}^{(j-1)}\right) \\
& \quad \vdots \\
& \theta_{p}^{(j)} \sim \pi\left(\theta_{p} \mid \theta_{1}^{(j)}, \theta_{2}^{(j)}, \ldots, \theta_{p-1}^{(j)}\right)
\end{aligned}
$$

(iii) mudamos a contador $j$ para $j+1$ e retornamos a (ii) até a convergência. 
Geman e Geman (1984) mostram que os valores geradós para $\theta_{i}, \quad i=1, \ldots, p$, convergem em distribuição para uma variável aleatória da distribuição $\pi(\theta) \operatorname{com} j \longrightarrow \infty$. 


\section{Apêndice B}

\section{ALGORITMO}

\section{METROPOLIS-HASTINGS}

Este algoritmo é usado quando as densidades condicionais não são dadas sob forma padronizada conhecida, como de uma Normal, Gama ou outras.

Para descrevermos este método consideremos uma distribuição $\pi$, com densidades condicionais não padronizadas, da qual desejamos gerar uma amostra através de uma cadeia de Markov. Nesse caso, devemos construir um núcłea de transição $p(\theta, \phi)$ de fạrma que $\pi$ seja a distribuição de equilíbrio da cadeia.

Uma forma simples de fazermos isso é através de cadeias onde o núcleo $p$ satisfaça a condição de reversibilidade $\pi(\theta) p(\theta, \phi)=\pi(\phi) p(\phi, \theta), \quad \forall(\theta, \phi)$, senda a condição suficiente para que $\pi$ seja a distribuição de equilíbrio da cadeia.

O núcleo $p(\theta, \phi)$ é constituído de dois elementos um núcleo de transição $q(\theta, \phi)$ e ụma probabilidade $\mathrm{a}(\theta, \phi)$ de forma que:

$$
p(\theta, \phi)=q(\theta, \phi) \mathrm{a}(\theta, \phi), \text { se } \phi \neq \theta .
$$

Portanto, o núcleo de transição define uma densidade $p(\theta, \cdot)$ para todos os valores diferentes de $\theta$. Consequentemente, resta uma probabilidade positiva da cadeia ficar em $\theta$ dada por,

$$
p(\theta, \theta)=1-\int q(\theta, \phi) \mathrm{a}(\theta, \phi) \partial \phi
$$

Essas duas formas podem ser agrupadas na expressão geral:

$$
p(\theta, A)=\int_{A} q(\theta, \phi) \mathrm{a}(\theta, \phi) \partial \phi+I(\theta \in A)\left[1-\int q(\theta, \phi) \mathrm{a}(\theta, \phi) \partial \phi\right] .
$$


Logo, o núcleo de transição define uma distribuição mista para o novo estado $\phi$ da cadeia. Para $\phi \neq 0$, essa distribuição tem densidade e para $\phi=0$, essa distribuição atriibui uma probabilidade positiva.

A expressão mais comumente citada para a probabilidade de aceitação é

$$
\mathrm{a}(\theta, \phi)=\min \left\{1, \frac{\pi(\phi) q(\phi, \theta)}{\pi(\theta) q(\theta, \phi)}\right\}
$$

Assim, a simulação de uma amostra $\pi$ usando a cadeia de Markov definida pela transição (B.1) pode ser esquematizada da seguinte forma:

(i) inicializamos o contador de iterações da cadeia $j=1$.e arbitremos o valor inicial $\theta^{(0)}$

(ii) movemos a cadeia para um novo valor $\phi$ gerado da densidade $q\left(\theta^{(j-1)},.\right)$;

(iii) calculamos a probabilidade de aceitação do movimento $\mathrm{a}\left(\theta^{(j-1)}, \phi\right)$ dada por (B.2). Se o movimento for aceito $\theta^{(j)}=\phi$. Se não for aceito, $\theta^{(j)}=\theta^{(j-1)}$ e a cadeia não se move;

(iv) mudamos o contador de $j$ para $j+1$ e retornamos a (ii) até a convergêpcia.

Realizamos a etapa (iii) após a geraçãa de uma quantidade uniforme $u$ independentes de todas as outras varíaveis. Se $u \leq \mathrm{a}$; o movimento é aceito e se $u>$ a o movimento não é permitido. O núcleo de transição $q$ define uma proposta de movimento que pode ou não ser confirmado por a. $\mathrm{O}$ sucesso do método depende de taxas de aceitação não muito baixas e de propostas fáceis de simular. O método substitui uma geração difícil de $\pi$ por várias gerações propostas de $(q)$. 


\section{Apêndice C}

\section{O CRITÉRIO DE}

\section{CONVERGENCIA DE GELMAN E}

\section{RUBFN (1992)}

Esta é uma forma simples de monitorar a convergência dos valores gerados para uma distribuição de equilíbrio.

O critério de convergência de Gelman e Rubin (1992) ébaseado em técnicas de análise de variância. Para o algoritmo devemos considerar pelo menos duas cadeias parajelas com valores iniciais, diferentes, amostrados de uma distribuição bem comportada. Após as cadeias atingirem estacionariedade digamos na t-ésima iteração, consideramos as realizações $\left(\theta_{j}, \theta_{j+h}, \ldots, \theta_{j+n h}\right), \quad j \geq t$ como uma amostra aleatória da distribuição desejada. Devemos assumir $h$ razoavelmente grande de tal forma que 2 valores sucessivos de $\theta$ sejam independentes.

Se cada sequência tem tamanho $2 n$, descartamos as primeiras $n$ iterações e consideramos as $n$ restantes. Para cada parâmetro de interesse, calculamos:

- a variância entre as cadeias:

$$
E=\frac{n}{m-1} \sum_{i=1}^{m}\left(\bar{\theta}_{i}-\bar{\theta}\right)^{2}
$$

- a variância dentro das cadeias:

$$
D=\frac{1}{m(n-1)} \sum_{i=1}^{m} \sum_{j=1}^{n}\left(\theta_{i}^{(j)}-\bar{\theta}_{i}\right)^{2}
$$


onde: $\bar{\theta}_{i}$ ê a média das oচsservaçōes dả $i$-êsìna cadéaja; e

$\bar{\theta}$ é a média $\operatorname{dos} \bar{\theta}_{i} \operatorname{com} i=1,2, \ldots, m$.

Então, a variância da distribuiçãa desejada-pode ser estimada por $\hat{\sigma}^{2}=\frac{n-1}{n} D+\frac{1}{n} E_{\xi}$ e a média por $\hat{\mu}=\overline{\boldsymbol{\theta}}$. Podemos melhorar na estimativa mais precisa da distribuiçāo desejậda, admitindo para a variabilidade amostral das estimativas, $\mu$ e $\hat{\sigma}^{2}$. O resultado é uma distribuição $t$ de Student aproximada para $\theta$ com centro $\hat{\mu}$, escala $\sqrt{\hat{V}}=\sqrt{\hat{\sigma}^{2}+E / m n}$ e graus de liberdade $d f=2 \hat{V}^{2} / \vartheta a ̂ r(\hat{V})$, ande

$$
\begin{aligned}
v \hat{a} r(\hat{V})= & \left(\frac{n-1}{n}\right)^{-2} \frac{1}{m} v \hat{a} r\left(s_{i}^{2}+\left(\frac{m+1}{m n}\right)^{2} \frac{2}{m-1} E+\right. \\
& +2 \frac{(m+1)(n-1)}{m n^{2}} \frac{m}{n}\left[\operatorname{cov} v\left(s_{i}^{2}, \bar{\theta}_{i}^{2}\right)-2 \bar{\theta} \operatorname{cov} v\left(s_{i}^{2}, \overline{\theta_{i}}\right)\right],
\end{aligned}
$$

onde as variâncias e covariâncias estimadàs são obtiđäs dós $m$ valores amostrais de $\bar{\theta}_{i} \mathrm{e}$ $s_{i}^{2} ; d f \longrightarrow \infty$ quando $n \longrightarrow \infty$.

Monitoramos a convergência dar simulação iterativa, calculando o fator de redução potencial dè escala estimado $\sqrt{\hat{R}}=\sqrt{(\hat{V} / D) d f /(d f-2)}$. A medida que $n$ cresce $(n \longrightarrow$ $\infty), R$ converge para 1 . $\hat{R}$ é a razão do estimạdor da variância atual para a variância dentro da cadeia com um fator que avalia a variância adicional da distribuição 't de Student'.

Desde que $\hat{R}$ esteja próximo de 1 , admitimos que a convergência ocorreu e as amoṣtras das iteraçães selecionadas sãa independentes e identicamente distribuídas (ver, Gelman \& Rubin, 1992). 


\section{Apêndice D}

\section{O CRITÉRIO DE}

\section{CONVERGÊNCIA DE GEWEKE}

\section{(1992)}

Este é critério mais simples de monitorar a convergência para uma distribuição de equilíbrio quando usamos uma única longa cadeia. Uma das grandes vantagens de se adotar uma única cadeia neste tipo de porcedimento de simulação é que tal cadeia devido ao seu grande tamanho, fica mais "próxima"da distribuição de equilíbrio, pois médias ergódicas são tanto melhores quanto maior a amostra.

O algoritmo pode ser explicado considerando os seguintes passos:

- Assume-se que a cadeia foi simulada por $m+n$ iterações.

- Sejä $\theta^{(j)}=\theta\left(X^{(j)}\right)$, para $j=1, \ldots, n$; uma funçãö feal.

- A trajetória $\theta^{(1)}, \theta^{(2)}, \ldots$;define uma série témporal è médias ergóticas para esta série podem ser calculadas.

As médias ergóticas são determinadas da seguinte forma:

$$
\hat{\theta}_{b}=\frac{1}{n_{b}} \sum_{j=m+1}^{m+n_{b}} \theta^{(j)}
$$

e

$$
\hat{\theta}_{a}=\frac{1}{n_{a}} \sum_{j=m+n \rightarrow n_{a}+1}^{m+n} \theta^{(j)}
$$


onde $n_{b}+n_{a}<n$. Uma sugestão é tomar $n_{a}=0.5 n$ e $n_{b}=0.1 n$.

À medida que $n$ cresce e as relações $\frac{n_{a}}{n}$ e $\frac{n_{b}}{n}$ são mantidas constantes, tern-se que :

$$
Z_{G}=\frac{\hat{\theta}_{a}-\hat{\theta}_{b}}{\sqrt{\hat{V} \operatorname{Var}\left(\theta_{a}\right)+\hat{V} \operatorname{ar}\left(\theta_{b}\right)}} \rightarrow N(\theta, 1)
$$

Assim, $Z_{G}$ não deve ser grande $(>2)$ se a convergência fói alcançada.

Graficamente, tem-se

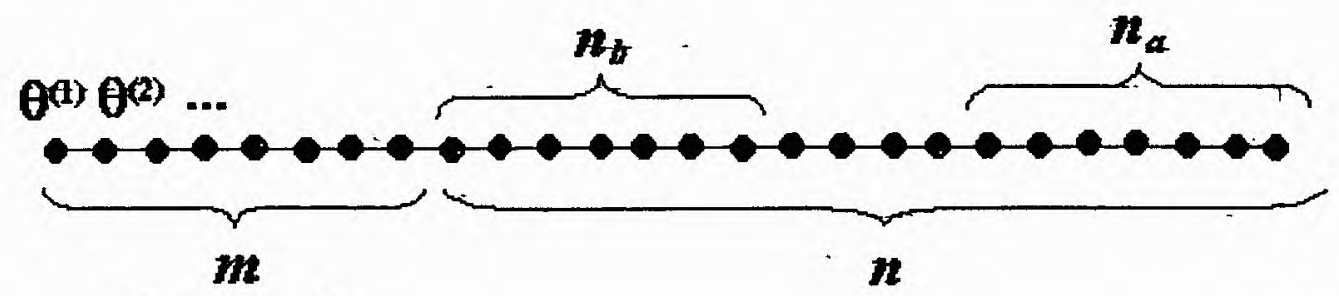

(hurn-in) 


\section{Apêndice E}

\section{UM PROGRAMA}

\section{COMPUTACIONAL}

Abaixo apresentamos um programa computacional em "Ox", referente ao algoritmo de Mistura de Distribuições Normais Multivariadas com Distribuição a Priori Conjugạda, citado no Capítulo 4.

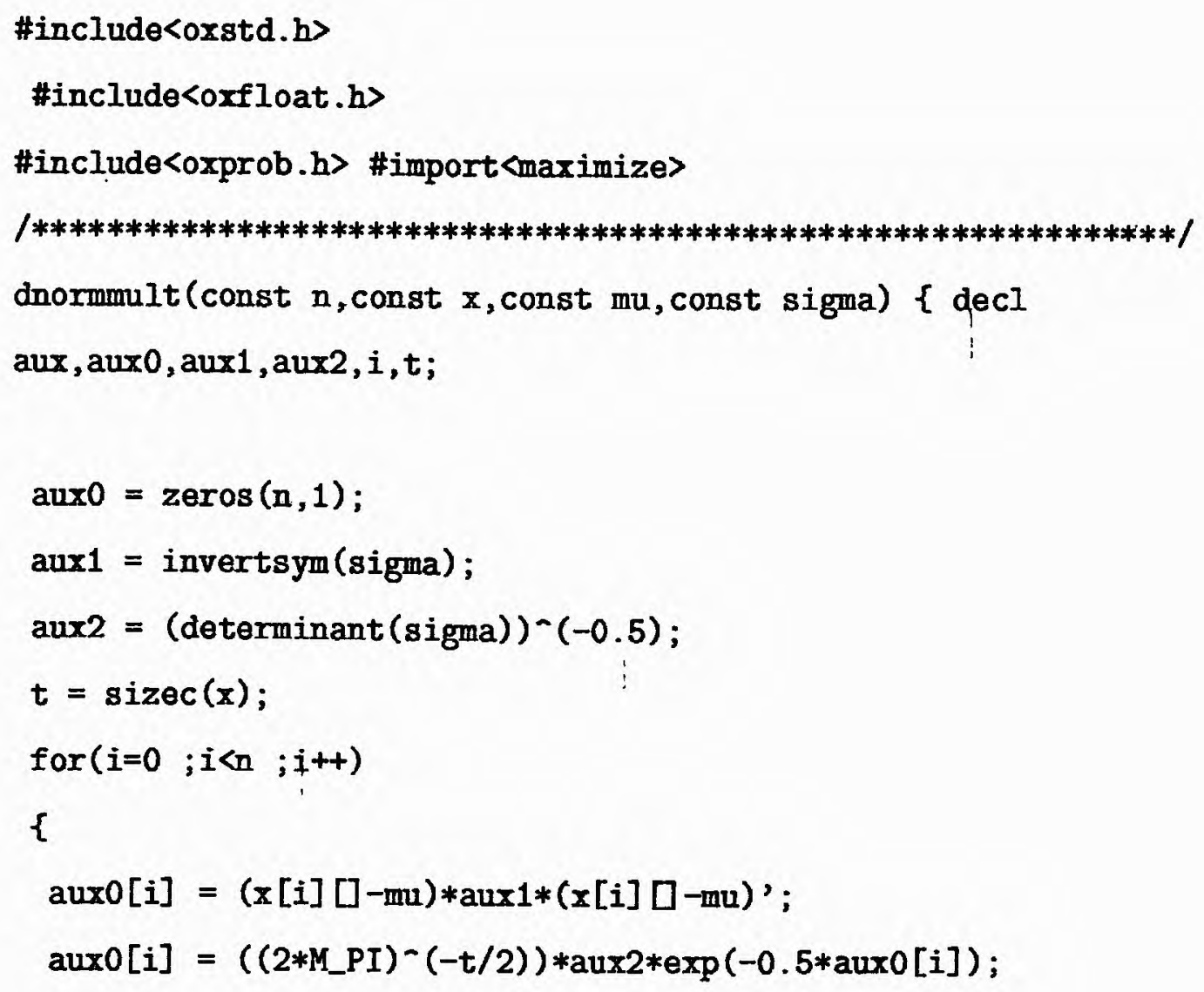




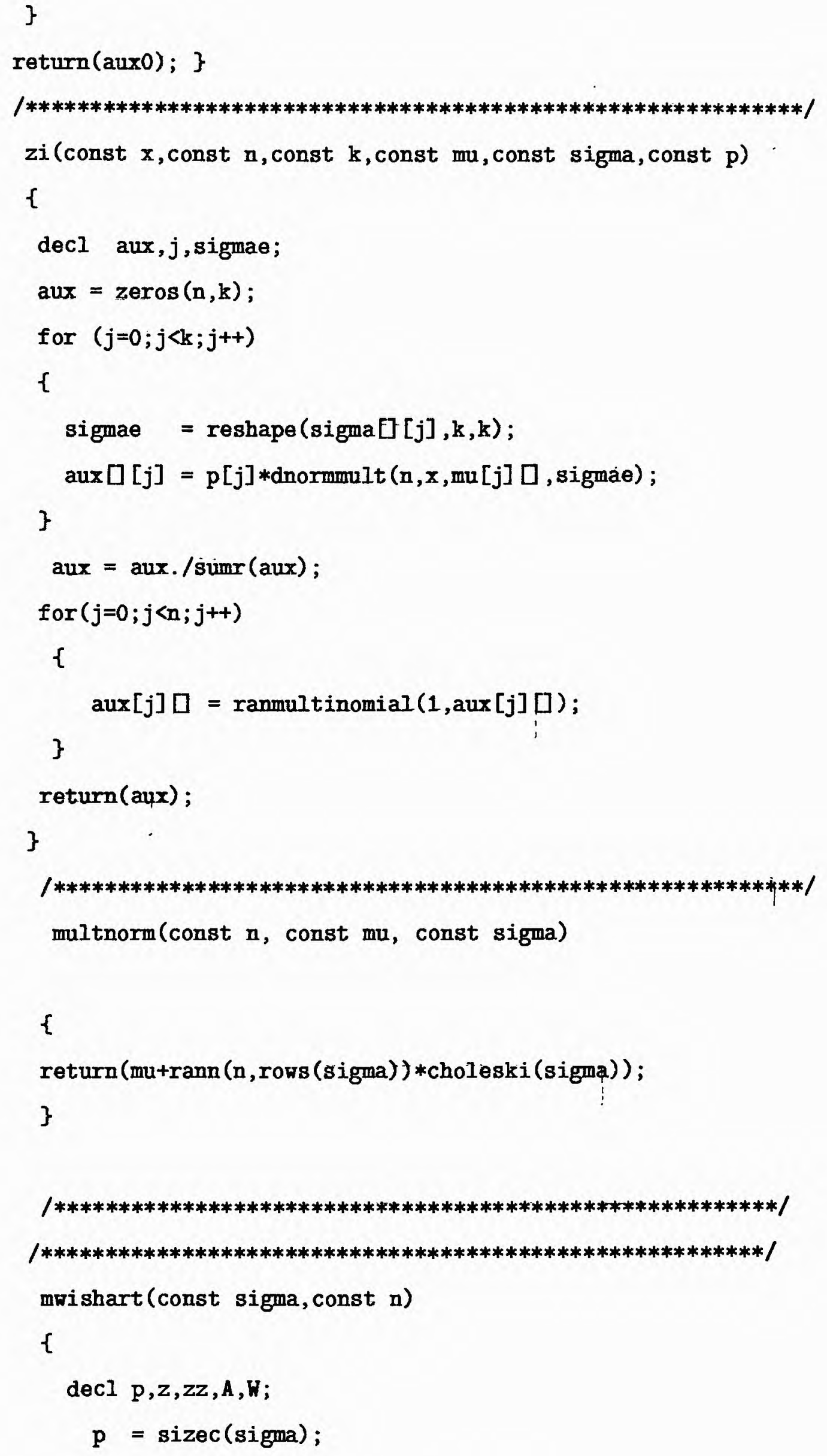




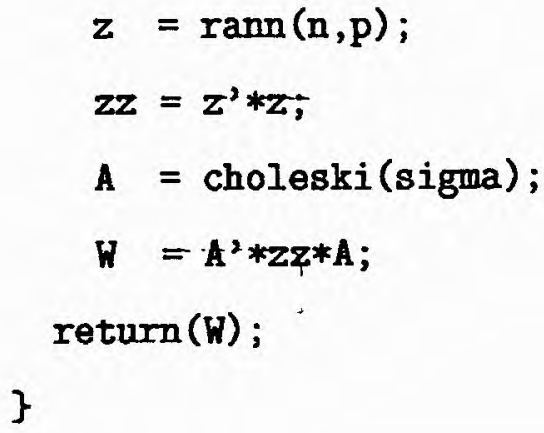




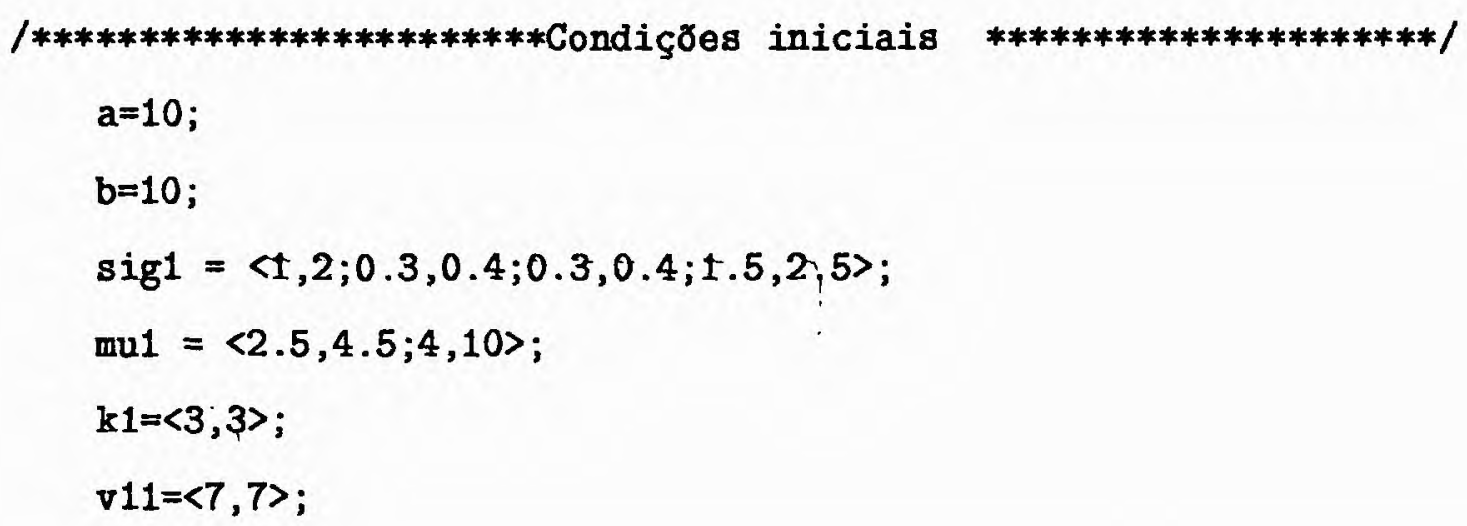




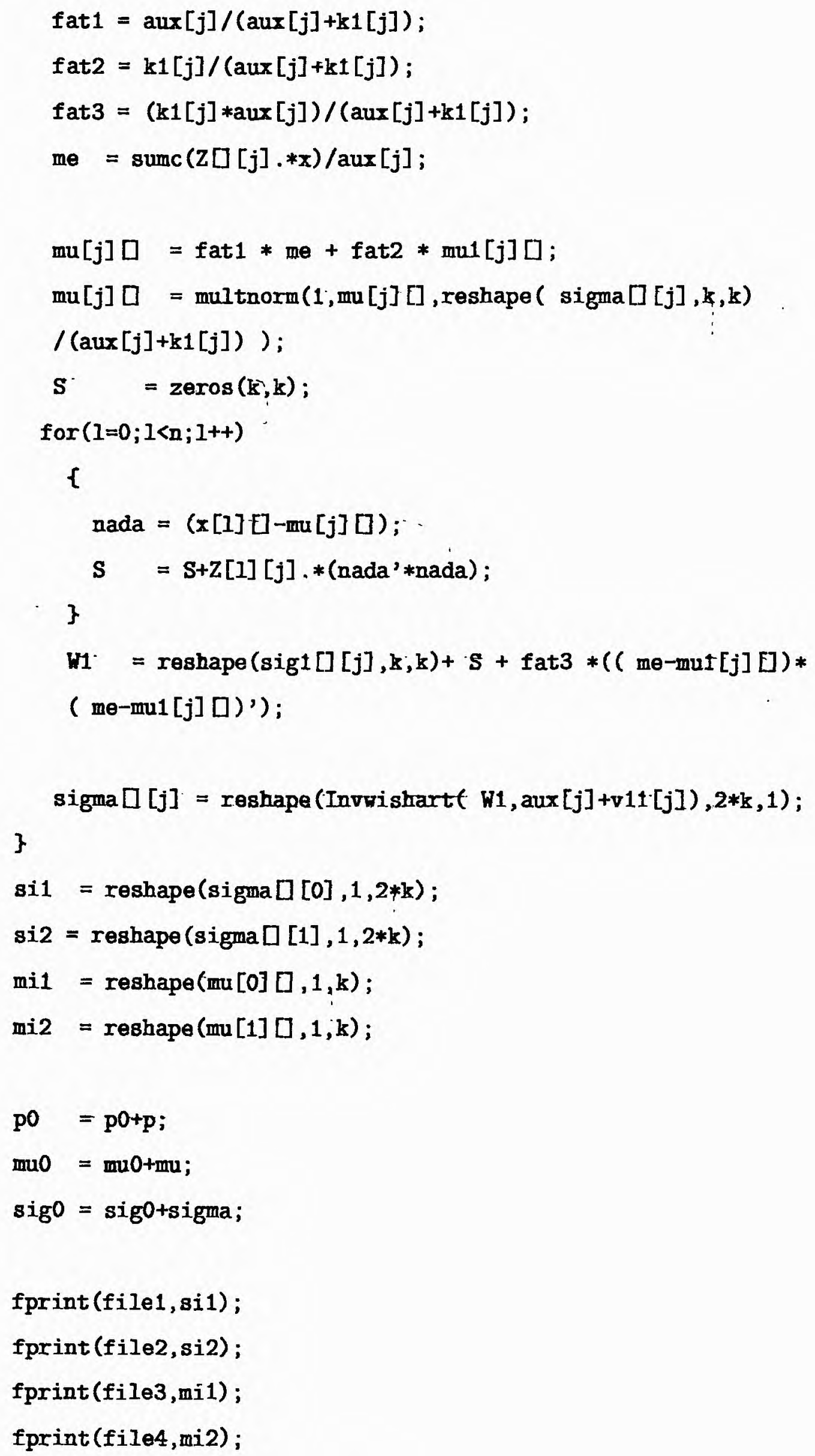


fprint (file5,p); 


\section{Referências Bibliográficas}

[1] Anderson, T: W. An Introduction to Multivariate Statistical Methods. John Wiley, New York, 1984.

[2] Box, G. E. P;Cox, D. R. An analysiys of transformations. J. R. Statist. Soc, B, n.2,v.26, pp. 211-252, 1964.

[3] Box, G. E. P.; Tiao, G. T. Bayesian Inference in. Statistical Analysis. Wiley Classics, New York, 1973.

[4] Cacoullos, T. Discriminante Analysis end Applications_Academic Press, New York, 1973.

[5] Doonik,J.A.. Object-Oriented.Matrix Programming Using Ox.,3rd ed. London: Timberlake Consultants Press and Oxford, 1999.

[6] Fisher,R. A.The Use of Multiple Measurements in Taxonomic Annals of Eugenics, 7, p. $179-188,1936$.

[7] Gelfand, A.; Smith, A. Sampling basead approaches to calculating marginal densities.Journal of the American Statistical Association, n.410, v.85, pp. 398-409, 1990.

[8] Gelman, A.; Rubin, B=D. Inference from iterative Simulation Using Multiple Sequences.Statistical Science, n.4, pp. 457-511, 1992.

[9] Gelman, R.; Carlin,J.B.; Stern, H.S. \& Rubin, D.B. Bayesian Data Analysis. Chapman and Hall.Gelman, 1995.

[10] Geweke, J. Evaluating the accuracy of sampling-based approaches to the calculation of posterior moments.In: Bayesian Statistics 4, J. M. Bernardo, A. F. M. Smith, A. 
P. Dawid, and J. O. Berger (eds.), pp. 169-193. New York: Oxford University Press, 1992.

[11] Goldstein, M;Dillon, W. R. Discriminant Analysis. Wiley, New York, 1978.

[12] Hosmer,.D. W.; Lemeshow,1989 Applied Logistic Regression.Wiley ,New York Wiley 1989.

[13] Huberty, C. J. Discriminant analysis.Rev. Educ. Res., v.45, pp. 543-593, 1975.

[14] Johnson, R. A.; Wichern, D. W. Applied Multivariate Statistical Analysis. Prentice Hall, New Jersey, 1982.

[15] Krzanowski, W. J. Discrimination and classification using both binary and continuous variables.Journal of the American Statistical Association, n.352, v.70; pp. $782-790,1975$.

[16] Lachenbruch, P. A. Discriminant Analysis. Hafner, New York, 1975a.

[17] Lachenbruch, P. A.; Goldstein, M. Discriminant-Analysis. Biometrics,..v.35, pp.69$85,1979$.

[18] Lachenbruch, P. A.; Mickey, M. R. Estimation of Error Rates in Discriminant Analysis. Technometrics, n.1, v.10; pp.1-11, 1968.

[19] Lavine, M.; West, M. A Bayesian method. for classification and discrimination.The Canadian Journal of Statistics, n.4, v.20, pp.451-461, 1992.

[20] Lee, E. T. Statistical Methods for Surviral Data Analysis. Awiley-Interscience, New York, 1992.

[21] McLachlan, G. J.; Krishnan, T. The EM Algorithm and Extensions. Wiley, New York, 1997.

[22] Press, S. J. Applied Multivariate Analysis. Holt,Rinehart, and Winston, New York, $1972 \mathrm{a}$.

[23] Prentice, R. L. Correlated Binary Regression with Covariate Specific to Each Binary Observation Biometrics,n.44,p1033-1048,1988. 


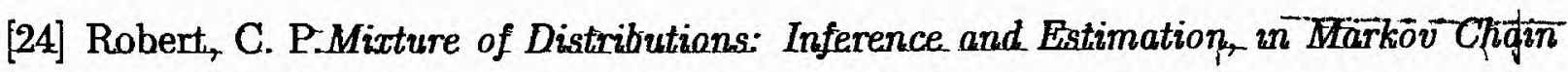
Monte Carlo in Practice, Chapman and Hall, Eondon, 1996. pp:441-464.

[25] Seber, G. A. F. Multivariate Observations. Wiley, New York, 1983.

[26] Smith, A. F. M.; Robert, G. O. Bayesian Computation via Gibbs Sampler and Related MCMC Methods. Jomal of the Royal Statistical Societ,B ,n.55, pp.3-24, 1993.

[27] Tanner, M.; Wong,W The calculations of.Posterior Distributions by Date Augmèntation. Journal of the American Statistical Association,82, p.528-550, 1987.

[28] Titterington, D.M.; Smith,A.F.M.;Makov,U.V Satistical Analysis of Finite Mixture Distributions. New York: John Wiley,1985. 